\title{
REAL TIME OPTIMAL CONTROL OF DISTRIBUTED-PARAMETER SYSTEMS
}

by

\author{
DEBAPRASAD DUTTA \\ B.Tech., National Institute of Technology, Durgapur, India, 2015
}

\author{
A thesis \\ presented to Ryerson University \\ in partial fulfillment of the \\ requirements for the degree of \\ Master of Applied Science \\ in the program of \\ Chemical Engineering
}

Toronto, Ontario, Canada, 2019

(C)Debaprasad Dutta, 2019 


\section{Author's Declaration}

Myself, Debaprasad Dutta, hereby declare that I am the sole author of this thesis. This is a true copy of the thesis, including any required final revisions, as accepted by my examiners. I authorize Ryerson University to lend this thesis to other institutions or individuals for the purpose of scholarly research. I further authorize Ryerson University to reproduce this thesis by photocopying or by other means, in total or in part, at the request of other institutions or individuals for the purpose of scholarly research. I understand that my thesis may be made electronically available to the public. 


\title{
Real Time Optimal Control of Distributed-Parameter Systems
}

\author{
Debaprasad Dutta \\ Master of Applied Science, 2019 \\ Department of Chemical Engineering, \\ Ryerson University
}

\begin{abstract}
An optimal state feedback control strategy is proposed for processes described by non-linear, distributed-parameter models. For different values of a given parameter susceptible to upsets, the strategy involves off-line computation of a repository of optimal open-loop control, state, and the gain needed for the feedback adjustment of control. The gain is determined by minimizing the perturbation of the objective functional, state and control due to an upset. When an upset is encountered in a running process, the repository is utilized to obtain the control adjustment required to steer the process to the new optimal state. The strategy is successfully applied to a highly non-linear, heavy oil recovery process with the state depending non-linearly on time and two spatial directions inside a moving boundary, and subject to pressure upsets. The results demonstrate that the proposed strategy is able to determine control adjustment with negligible time delay, and navigate the process to the new optimal state when disturbed by a pressure upset.
\end{abstract}




\section{Acknowledgments}

My sincere most gratitude and reverence to my supervisor Professor Simant Ranjan Upreti for his excellent supervision and inspiration throughout this thesis. Whenever I faced problem on my computer programming in $\mathrm{C}++$ or in the $\mathrm{ATE}_{\mathrm{E}} \mathrm{X}$ document writing or in Inkscape technical drawings or in Aymptote mathematical drawings, he sat with me and clarified all my queries, which I have never experienced anywhere. From his astute instructions and guidance, I learned not only the arts of presentation but also I have acquired erudite set of skills about managing new software applications and most importantly the way of channelizing thought-process.

I am very grateful for the invaluable assistance of faculty members and technologists in the Chemical Engineering Department of Ryerson University, especially those provided by Ali Hemmati, Daniel Boothe, and Tondar Tajrobehkar during the equipment manufacture and system setup.

I also would like to thank the Department of Chemical Engineering and the Yeates School of Graduate Studies of Ryerson University for their support in terms of department facilities, research stipend, and scholarships.

I would also like to thank Goretti Praticante, Jessica Miniaci and Tatjana Grabeljsek in the Department of Chemical Engineering. 


\section{Dedication}

My sincere most reverence and love to my mother Rinka Dutta and my father Swapan

Kumar Dutta for their affection and support throughout my life. Secondly, my mentor, well-wisher, who motivated me to work hard, my supervisor Dr. Simant R. Upreti. 


\section{Contents}

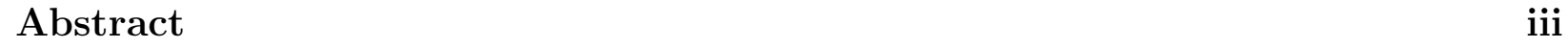

$\begin{array}{ll}\text { List of Tables } & \text { ix }\end{array}$

List of Figures $\quad \mathrm{x}$

Nomenclature xii

$\begin{array}{llr}1 & \text { Introduction } & 1\end{array}$

1.1 Control Theory Fundamentals . . . . . . . . . . . . . . . . . . . . 1

1.2 Review of Different Control Frameworks . . . . . . . . . . . . . . . . . 2

1.3 Literature Survey $\ldots \ldots \ldots \ldots$. . . . . . . . . . . . . . . . . . 7

1.3 .1 Selection of Control Strategy $\ldots \ldots \ldots \ldots$

$1.3 .2 \quad$ State Feedback Law: Advances on Riccati based approach $\ldots . . . .10$

1.4 Novelty of the Thesis $\ldots \ldots \ldots \ldots$

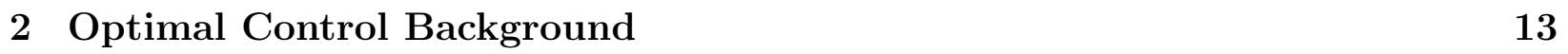

2.1 Problem Statement . . . . . . . . . . . . . . . . . . . . . . . . . . . . . . 13

$2.2 \quad$ Augmented Functional $\ldots \ldots \ldots \ldots \ldots$

$2.3 \quad$ Analysis of Optimal Control . . . . . . . . . . . . . . . . . . 15

$2.4 \quad$ Hamiltonian Based Approach $\ldots \ldots \ldots$. . . . . . . . . . . . 16

2.5 Pontryagin's Minimum Principle $\ldots \ldots \ldots \ldots$. . . . . . . . . . . . 17

2.6 Summary of Necessary Conditions $\ldots \ldots \ldots \ldots$ 
$2.7 \quad$ Solution of Optimal Control Problem $\ldots \ldots \ldots \ldots$

\begin{tabular}{|lll}
\hline 3 & Theoretical development & 21
\end{tabular}

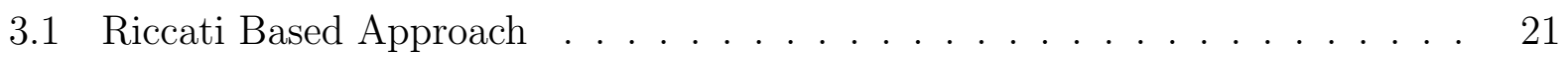

3.2 Basis of Closed Loop Control $\ldots \ldots \ldots$

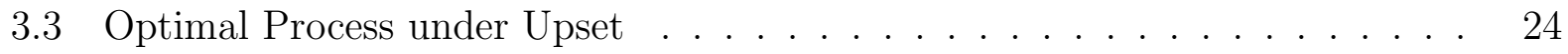

3.4 Incorporation of State Feedback $\ldots \ldots \ldots \ldots \ldots$

$3.5 \quad$ Feedback Optimal Control Strategy $\ldots \ldots \ldots \ldots$

\begin{tabular}{llr}
\hline & Application & 29
\end{tabular}

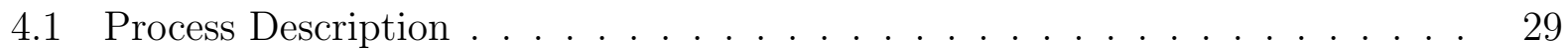

$4.1 .1 \quad$ Experimental Set-up . . . . . . . . . . . . . . . . . . . . . . . . . . 29

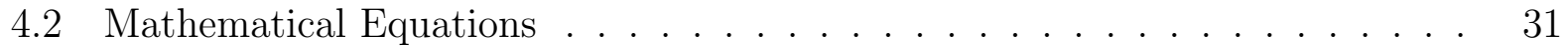

4.2 .1 Process Model . . . . . . . . . . . . . . . . . . . . . . . . . . . . . . . 32

4.2 .2 Objective Functional $\ldots \ldots \ldots \ldots \ldots$

$4.2 .3 \quad$ Necessary Conditions of Optimality . . . . . . . . . . . . . . . . 38

$4.2 .4 \quad$ Closed Loop Optimal Control Framework. . . . . . . . . . . . . . . 40

4.3 Development of Computer Program . . . . . . . . . . . . . . . . . . . 41

4.4 Simulation Results . . . . . . . . . . . . . . . . . . . . . . . . . . . . . 43

$4.4 .1 \quad$ Open Loop Simulation Results . . . . . . . . . . . . . . . . . . . . 44

4.4 .2 Closed Loop Simulation Results . . . . . . . . . . . . . . . 55

$\begin{array}{lll}5 \text { Conclusions } & 61\end{array}$

$5.1 \quad$ Future Work . . . . . . . . . . . . . . . . . . . . . . . . . . . . . . . 62

\begin{tabular}{ll}
\hline Appendices & 63
\end{tabular}

\begin{tabular}{|lll}
\hline Appendix A Experimental Details & 64
\end{tabular}

A.1 Apparatus for Experimental Investigation. . . . . . . . . . . . . . . 64

A.2 Description of Various Parts . . . . . . . . . . . . . . . . . 66 
A.3 Determination of Interfacial Concentration . . . . . . . . . . . . . . 73

\begin{tabular}{ll}
\hline Appendix B Permeability Calculation & 76
\end{tabular}

\begin{tabular}{|ll}
\hline Appendix C Viscosity and Diffusivity Data & 77
\end{tabular}

C.1 Viscosity Data . . . . . . . . . . . . . . . . . . . . . . . . . . . 77

C.2 Diffusivity Data . . . . . . . . . . . . . . . . . . . . . 78

\begin{tabular}{|l|l}
\hline Appendix D Experimental Results & 81
\end{tabular}

D.1 Experimental Result - Solubility Data . . . . . . . . . . . . . . . . . 81

D.2 Experimental Result - Heavy Oil Mass . . . . . . . . . . . . . . . . . 82

\begin{tabular}{|ll|}
\hline Appendix E & Derivation of Process Model Equations \\
\hline
\end{tabular}

E.1 Mathematical Modeling for Mass Fraction . . . . . . . . . . . . . . . . . 84

E.2 Mathematical Modeling for Energy _. . . . . . . . . . . . . . . . . 85

E.3 Mathematical Modeling for Momentum . . . . . . . . . . . . . . . 87

\begin{tabular}{|lll}
\hline Appendix F & Model Simulation Results & 88
\end{tabular}

\begin{tabular}{|l|l|}
\hline Appendix G Derivation of Necessary Conditions & 91
\end{tabular}

\begin{tabular}{lr}
\hline Bibliography & 114
\end{tabular} 


\section{List of Tables}

1.1 Key Features of Different Control Frameworks . . . . . . . . . . . . . . . . 6

4.1 Matrix Dimensions . . . . . . . . . . . . . . . . . . . . . . . . 41

4.2 Simulation Parameters . . . . . . . . . . . . . . . . . . . . . . . . . . . 45

4.3 Parameters used in Computations . . . . . . . . . . . . . . . . . . 46

4.4 Objective functionals for different initial guesses - set 1 . . . . . . . . . . . . 49

4.5 Objective functionals for different initial guesses - set 2 . . . . . . . . . . . 50

4.6 Grid point analysis . . . . . . . . . . . . . . . . . . . . 51

4.7 Open Loop Results . . . . . . . . . . . . . . . . . . . . . . . . . . . . . 52

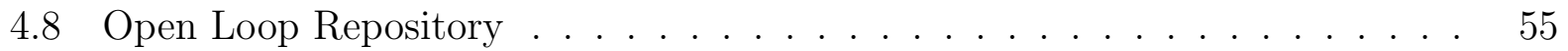




\section{List of Figures}

1.1 Basic Structure of a IMC $\ldots \ldots \ldots \ldots$. . . . . . . . . . . . . . 4

$3.1 \quad$ Closed Loop Optimal Control Strategy $\ldots \ldots \ldots$

$4.1 \quad$ Schematic of Lab-scale Setup $\ldots \ldots$. . . . . . . . . . . . . . . . . . 30

4.2 Cylindrical Geometry $\ldots \ldots \ldots$

$4.3 \quad$ Initial guess for control function - set $1 . \ldots \ldots \ldots$. . . . . . . 47

4.4 Initial guess for control function - set $2 . \ldots \ldots \ldots$

$4.5 \quad$ Results for $24.5 \mathrm{psi}, 50 \mathrm{psi}$ and $74.5 \mathrm{psi} . \ldots \ldots \ldots \ldots$

4.6 Results for 43.5 psi and 58.5 psi. . . . . . . . . . . . . . 50

4.7 Convergence of objective functional. $\ldots \ldots \ldots \ldots \ldots$

4.8 Optimal temperature policies at different pressures. . . . . . . . . . . . 54

4.9 Closed Loop Result: Single upset within known bounds after $23 \mathrm{~min} . \quad$. . . . 57

4.10 Closed Loop Result: Single upset within known bounds after $73 \mathrm{~min} . \quad$. . . . 58

4.11 Closed Loop Result: Multiple upsets. . . . . . . . . . . . . . . . . . . . 59

4.12 Optimal temperature at grid points $i=2$ and $j=3 . \ldots \ldots$. . . . 60

4.13 Error Analysis at Different Grid Points. . . . . . . . . . . . . . . . 60 


\title{
Nomenclature
}

\author{
$A \quad$ area, $\mathrm{m}^{2}$ \\ $D \quad$ diffusivity of gas, $\mathrm{m}^{2} \mathrm{~s}^{-1}$ \\ $g \quad$ gravity, $\mathrm{m} \mathrm{s}^{-2}$ \\ I objective functional \\ $J$ augmented functional \\ $K \quad$ permeability \\ $K_{\mathrm{r}} \quad$ relative permeability \\ $C_{\mathrm{p}} \quad$ specific heat, $\mathrm{J} \mathrm{kg}^{-1} \mathrm{~K}^{-1}$ \\ $k$ thermal conductivity, $\mathrm{J} \mathrm{m}^{-1} \mathrm{~s}^{-1} \mathrm{~K}^{-1}$ \\ $\dot{m}$ heavy oil mass flow rate, $\mathrm{kg} \mathrm{s}^{-1}$ \\ $T \quad$ system temperature, $\mathrm{K}$ \\ $\bar{T} \quad$ average system temperature, $\mathrm{K}$ \\ $T_{\text {int }}$ interfacial temperature, $\mathrm{K}$ \\ $w \quad$ gas mass fraction \\ $\bar{w} \quad$ average gas mass fraction \\ $w_{\text {int }}$ interfacial gas mass fraction
}


$r \quad$ distance along radial direction, $\mathrm{m}$

$z \quad$ distance along radial direction, $\mathrm{m}$

$t$ time, $\mathrm{s}$

$t_{\mathrm{f}} \quad$ final time, $\mathrm{s}$

$v \quad$ Darcy velocity, $\mathrm{m} \mathrm{s}^{-1}$

$v_{0} \quad$ Darcy velocity at $z=Z_{0}, \mathrm{~m} \mathrm{~s}^{-1}$

$Z_{0} \quad$ initial height of the physical model, $\mathrm{m}$

$\delta x \quad$ variational state (temperature, gas mass fraction, axial distance)

$\delta \bar{x} \quad$ variational average state (temperature, gas mass fraction, axial distance)

$\delta x_{\text {int }}$ variational interfacial state (temperature, gas mass fraction)

$T_{\text {int }}^{\text {new }}$ updated interfacial temperature at each iteration, $\mathrm{K}$

$H \quad$ hamiltonian

$\triangle r \quad$ distance between successive grid points along the radial direction, $\mathrm{m}$

$\triangle z \quad$ distance between successive grid points along the axial direction, $\mathrm{m}$

u control vector

y state vector

$\hat{\mathbf{z}} \quad$ optimal vector

f constraint vector

S Riccati matrix

K gain matrix 


\section{Greek Letters}

$\begin{array}{ll}\rho & \text { oil density, } \mathrm{kg} \mathrm{m}^{-3} \\ \phi & \text { medium porosity } \\ \mu & \text { viscosity of heavy oil, } \mathrm{kg} \mathrm{m}^{-1} \mathrm{~s}^{-1} \\ \bar{\mu} & \text { average viscosity of heavy oil at } z=Z_{0}, \mathrm{~kg} \mathrm{~m}^{-1} \mathrm{~s}^{-1} \\ \gamma & \text { thermal diffusivity of the medium, } \mathrm{m}^{2} \mathrm{~s}^{-1} \\ \lambda_{i} & \text { co-state vectors } \forall i=1,2 \text { and } 3\end{array}$

\section{Abbreviations}

PID

proportional-integral-derivative

IMC

internal model control

MHPC

model heuristic predictive control

$\mathrm{DMC}$

dynamic matrix control

GPC

generalized predictive control

EMPC

explicit model predictive control

NMPC

non-linear model predictive control

EOR

enhanced oil recovery

HTWC

heater tape with controller

LabVIEW

laboratory virtual instrument engineering workbench

PVT

pressure vessel temperature 


\section{Chapter 1}

\section{Introduction}

In this introductory chapter, we briefly discuss about control theory fundamentals and describe different types of highly practiced control frameworks. Then we perform a literature survey on the cutting edge closed loop control practices and finally, we conclude with the novelty of our approach.

\subsection{Control Theory Fundamentals}

Control theory in Systems Engineering is a subfield of mathematics that deals with the control of continuously operating dynamic engineering processes and machines. The prime objective is to develop a detailed mathematical model and derive an optimum control action so that the process can be effectively controlled without any delay or overshoot along with ensuring system stability. To do this, a controller is mandated that navigates the controlled process variable and compares it with the optimum pre-determined set point value. The difference between this actual process variable and the desired set point value is called the error signal and it is applied as feedback to generate a control action to bridge the gap between these variables [1]. Theoretical basis of process control for various plant operations was first described by Edward Routh [2, Charles Sturm and Adolf Hurwitz who all contributed to the development of control stability criteria as well [3]. From 1922 onwards, the development of PID (proportional-integral-derivative) control theory was initiated by Nicolas Minorsky 
[4]. Although, major application of control theory is in systems engineering, that deals with the design of process control systems for industrial operations, other applications range far beyond this.

\section{Basic Advantage of Closed Loop Control}

Fundamentally, there are two types of control strategies: open loop control and closed loop (feedback) control. In open loop control mode, controller action is independent of the process output (controlled process variable). In closed loop control, the control action from the controller is necessarily dependent on the process output to monitor the error signal; based on this error, necessary corrective measure is taken by the finite control element in order to minimize the deviation and drive the process to desired operating point [5]. A closed loop controller therefore has a feedback loop which ensures the controller exerting a control action to give a process output the same as the set point.

For this reason, closed loop controllers are also referred as feedback controllers. Open loop control is an admissible choice when low cost is a preference, output varies rarely or not at all and no quantitative measurement (dynamic) is possible or process disturbances are highly rare. On the other hand, closed-loop control offers significant process navigation as a process can be maintained at desired optimum point within a given accuracy. Moreover, Corrections to process disturbances can be automated and the problems associated with instability can be addressed [6]. It is noteworthy that, closed loop control strategies are highly reliant on open loop results and researchers congregate various open loop optimization parameters to develop their feedback control algorithms [3, 5, 6].

\subsection{Review of Different Control Frameworks}

Feedback control frameworks are indispensable for a desired output for most of the large scale industrial operations. In this section, we discuss about different types of feedback controllers and review their performances for any flow process. 


\section{PID (Proportional-Integral-Derivative) Control Framework}

PID (proportional-integral-derivative) controllers are straightforward in principle, uncomplicated to tune, and is still extensively used in industrial process control [7]. Flexibility in parameter tuning makes it more user friendly towards plant operators and engineers; thus, it is being used in most of the simple and linear process applications. Tuning works well (for an analogue controller) a linear system having monotonic response [8]. Many methods are available in the literature to propose a perfect PID such as Ziegler-Nichols tuning, Cohen-Coon tuning and CHR tuning. Ziegler-Nichols tuning generate PID parameters such as controller path gain $\left(k_{\mathrm{p}}\right)$, integral $\left(T_{\mathrm{i}}\right)$ and derivative time $\left(T_{\mathrm{d}}\right)$ constants using period and frequency derived from Bode frequency diagrams [6, 8]. Cohen-Coon method rectifies sluggish, steady state response generated by Ziegler-Nichols method having large process delay; but this method is appropriate is mostly for first order processes [8]. CHR (Chien, Hrones and Reswich) method is a modification of Ziegler-Nichols tuning method using $0 \%$ overshoot criteria; as a result, the gain and the derivative time are lesser but the integral time is larger [9].

Even though many advanced process control mechanisms are based on a PID control algorithm, the same cannot effectively navigate practical production process with model non-linearities and time varying uncertainties [10].

\section{IMC (Internal Model Control) Control Framework}

A central concept of IMC (internal model control) states that control can be achieved only if the control system involves, either implicitly or explicitly, some mathematical formulation of the process to be controlled [11. In the basic IMC structure, as shown in Figure 1.1, a replica of plant model is fed back to the controller and we obtain a new controller; this new controller is advantageous in many ways. As described by Garcia et al.[12], the advantages are in terms of dual stability, perfect control and zero offset. The IMC structure offers these aforementioned advantages only when the plant model is perfect; but in reality, physical systems are non-linear. 


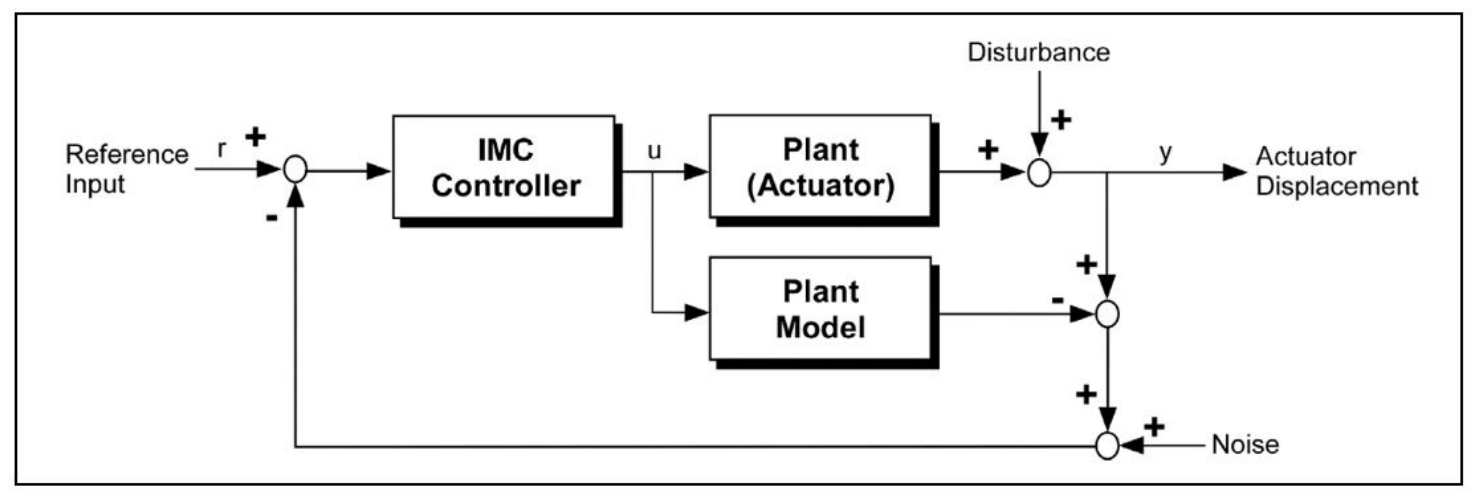

Figure 1.1: Basic Structure of a IMC

The selection of the invert plant model as a compensator can create instability even in the presence of an infinitesimal plant-model mismatch [11]. Thus, tuning, model non-linearity and robustness are major concerns associated with a IMC framework [13, 14].

\section{MHPC (Model Heuristic Predictive Control) Control Framework}

From mid-70s, several industrialists and academic practitioners started reporting effectiveness of computer-model based control in controlling various processing units in petrochemical refineries [15]. Richalet et al. [16] introduced a method called model heuristic predictive control (MHPC) and reported successful applications to large-scale industrial processes. MHPC employed a finite impulse response model and a reference trajectory (a path that describes how each controlled variable should return from the current status to the desired setpoint) along with coincidence points (the points in the prediction horizon where the output needs to be on the pre-specified reference trajectory). Hence, the main tuning knob for MHPC are the time constants of the reference trajectories. Process delays reduce the efficacy of this framework in some applications.

\section{DMC (Dynamic Matrix Control) Control Framework}

Charlie et al. [17] generated tremendous interest after reporting the use of a multivariable model-based control algorithm termed as dynamic matrix control (DMC) in the 1979 AIChE Annual Meeting and the 1980 Joint Automatic Control Conference. DMC utilizes 
a truncated step response model and least squares minimization of errors with respect to a constant setpoint. For DMC, main tuning knobs are the parameters in the least squares minimization. Due to significantly less barrier in terms of real time implementation, the use of these model-based computer control spread rapidly across petrochemical plants in the Western industries. This was the basis of the modern day control techniques collectively known as model predictive control (MPC), still high CPU time and robustness were major issues [15].

\section{GPC (Generalized Predictive Control) Control Framework}

Independent from the industrial research and development wing, the adaptive process control (control method utilized by a controller that must adapt to a controlled system with parameters which may vary, or are initially uncertain) community witnessed a rise of its own version of MPC named as generalized predictive control (GPC) [18]. While DMC was envisaged to deal with multivariable constrained control problems mainly for the petrochemical industries, GPC was proposed to offer an alternative to the self-tuning regulators, primarily to overcome robustness issues. Similar to adaptive control methodology, GPC utilized a transfer function model in the stochastic framework [15, 19]. Application of the same to multivariable control problems and inclusion of constraints became an issue. Though regarded to be theoretically more comprehensive than DMC and its contemporaries, GPC went highly unnoticed by the researchers [15].

\section{MPC (Model Predictive Control) Control Framework}

MPC (model predictive control) is a multivariable control algorithm that uses an internal dynamic process model, history of past control measures and finally, an optimization cost function over the receding prediction horizon [15]. Based on the structure of the associated model and the cost function, different types of MPC frameworks are available in the literature. Non-linear MPC (NMPC or online-MPC) is characterized by the use of non-linear system models and the numerical solution is typically based on direct optimal control meth- 
ods using Newton-type optimization schemes, in one of the variants: direct single shooting, direct multiple shooting methods or direct collocation [20]. Though it is used in the commercial scale applications most intensively, still it has limitations in terms of slow sampling rate, high CPU time and extensive computation [21].

Table 1.1: Key Features of Different Control Frameworks

\begin{tabular}{|l|l|l|}
\hline $\begin{array}{l}\text { Control } \\
\text { Framework }\end{array}$ & Benefits & Limitations \\
\hline PID & Flexible and user-friendly & Model non-linearity \\
\hline IMC & $\begin{array}{l}\text { Perfect process control with zero } \\
\text { offset and easy to implement }\end{array}$ & $\begin{array}{l}\text { Model non-linearity and im- } \\
\text { perfections }\end{array}$ \\
\hline MHPC & $\begin{array}{l}\text { Flexible in terms of parametric } \\
\text { tuning and quick control }\end{array}$ & Process delays \\
\hline DMC & $\begin{array}{l}\text { Comprehensive process control } \\
\text { and user-friendly applications }\end{array}$ & $\begin{array}{l}\text { High CPU time and robust- } \\
\text { ness }\end{array}$ \\
\hline GPC & $\begin{array}{l}\text { Applicable to multivariable con- } \\
\text { strained parameter problems }\end{array}$ & Robustness \\
\hline MPC & $\begin{array}{l}\text { Wide range of applications - deals } \\
\text { with model non-linearities and } \\
\text { multivariable problems }\end{array}$ & $\begin{array}{l}\text { High CPU time and compu- } \\
\text { tational overhead }\end{array}$ \\
\hline
\end{tabular}

In comparison to online-MPC, explicit-MPC (EPMC) is recognized for fast evaluation of the control law [22]. In this parametric programming technique, the control law (piecewise affine function - PWA) is solved offline and the coefficients are stored for each control region (convex polytopes) of the state space [23]. Thereafter, the optimal control action is determined in two steps: first, the region containing the current state is evaluated and then, the PWA is evaluated using the stored coefficients. In case, there is a growth in number of control regions, it becomes computationally intensive due to increment in controller memory 
requirements [24]. Various robustification techniques are also reported for MPC frameworks, out of which, multi-stage MPC is the most practiced one; but the major drawback is often associated with the uncertainties in the prediction horizon [25].

Table 1.1 summarizes important advantages and disadvantages of different closed loop control frameworks.

\subsection{Literature Survey}

Optimal control theory engages with the problem of finding a control law for a given system or a process such that a certain optimality criterion is satisfied. A control problem includes an objective functional involving state and control variables. Besides, an optimal control is a set of differential equations describing the paths of the control variables that can effectively optimize the objective functional. This optimal control necessary conditions can be derived using Pontryagin's principle (described in Chapter 2) or by solving the Hamilton-Jacobi-Bellman equation (a sufficient condition).

\section{Limitations of Open Loop Optimal Control}

Open loop optimal control policies are capable enough to stimulate multifarious objective functionals in terms of mass or revenue but in the present global scenario, the basic challenge in terms of field scale application is inherently associated with the projection and maintenance of the optimal state in presence of system or operational upsets; in other words, the developed open-loop strategies lack direct control on the process that is performing at its optimality. Open-loop control algorithm generally produces a sequence of optimal state signal for deterministic dynamics having zero perturbations. However in case the system leaves an optimal trajectory due to unavoidable modelling imperfections, it needs to be mended with the aid of a hand-tuned PID (proportional-integral-derivative) controller. This operation often leads to suboptimal behaviour as the error feedback has not been counted in optimization calculations [26]. Hence, a feedback strategy needs to be amended for operating the process at a desired optimal condition even when there is a tweak in the boundary conditions. 


\subsubsection{Selection of Control Strategy}

Several controller design methods and control algorithms are briefly described in Section 1.2. Out of them, Model predictive control (MPC) is the most sophisticated version of advanced process control. Three decades have passed since milestone publications from several industries and academic institutions on model predictive control (MPC). The first decade is characterized by the fast-paced industrial adoption of the technology, mostly in the refining and petrochemical sectors, that incited much interest and also confusion among the academicians [27, 28. The second decade reviewed a number of advances in comprehending the MPC from a control theoretician's viewpoint, which included state-space interpretations and stability proofs [29]. These theoretical developments contributed to the furnishing of the second generation of commercial software [30]. The third decade's main focus has been on the development of fast-paced MPC, a term chosen to collectively describe various endeavors to bring orders-of-magnitude improvement in the efficiency of the real time and online computation so that the technology can be applied to systems mandating very fast and accurate sampling rates [31]. Throughout the three decades of the development, theory and practice supported each other quite effectively, a primary reason for the fast and steady rise of the technology [32].

\section{Advantages and Disadvantages of NMPC}

Recent studies [33, 34, 35, have shown that non-linear dynamic optimization is the basis of model (non-linear) predictive control (NMPC) and has a crucial role in terms of real time implementation of a feedback control scheme. Adetola et al. [36] analyzed the effectiveness of NMPC and from their studies it is concluded that NMPC can address multivariable constrained non-linear systems, can encompass multifarious performance criteria and it also possesses a flexible structure for real-time optimizing control. Studies of De Souza et al. [34] and Huang et al. [35] also substantiates above inferences. Moreover, Aydin et al. [37] showcased that shrinking-horizon NMPC has potential to optimally operate semi-batch processes where the optimization problem is solved until the final time with a precise estimation of 
system states. In spite of all these beneficial factors, an inherent certain computational time often leads to non-negligible feedback delay in a closed-loop control operation. This CPU time is always associated with the solution of the corresponding non-convex optimal control problems in real-time mode. Gros et al. [38] demonstrated that this delay often results in suboptimal solution, or worse, infeasible operation. Hence, reduction of CPU time gains substantial importance and inevitably, it became an open research field both in academia and industrial operations. Wolf et al. [39] showcased a deep review of the broad class of fast computational methods for NMPC (suboptimal, explicit, hierarchical and sensitivity based).

\section{Feedback Law: An Alternative Approach}

Alternatively, stable optimal performance can only be accomplished by fabricating an optimal feedback law that generates a mapping from states to control actions by employing all available optimal process information. In such optimal feedback control scheme, there is hardly any separation between the desired trajectory and the real time trail for the completion of a given assignment [40]. In addition, researchers directly seek to deduce gain parameter values of a feedback controller which in turn produce an optimal mapping from state to control responses and are explicitly governed by a linearized control law. The notion of transformed linearized control law has better potential in terms of control robustness as it alleviates computational intricacies associated with model non-linearity.

This paper concentrates on the development and implementation of linearized control mechanism that effectively correlates optimal values of system state at different magnitudes of pressure to the corresponding optimal control with the aid of gain parameters. These gain parameters are inherently coupled with the optimal values of Riccati elements, state and co-state variables as a function of time. Optimal solutions of Riccati elements are eventually deduced from perturbation equations in terms of state and co-state variables. Thus entire computational overload could be dealt in off-line mode and we generate a repository in terms of optimal policy, optimal state and optimal gain at different boundary conditions. In the real time mode, we have utilized this repository and state feedback control law to 
take necessary control adjustment through simple interpolation in order to steer the process to optimality at the tweaked boundary condition. This interpolation mechanism, based on known process information, effectively addresses the aforementioned computational delay.

\subsubsection{State Feedback Law: Advances on Riccati based approach}

Optimal feedback controllers incorporate three crucial attributes. They are governed by an explicitly defined objective functional encompassing all relevant process variables along with their relative weighting. Besides, they can control mapping of multiple input signals onto more than one output signal and mandate precise estimation of system state. Associated errors are rectified by the controller in case they adversely affect process performance, otherwise can be neglected (as per minimum intervention principle that states that the deviations from the desired trajectory needs to be corrected only when they interfere with process performance). This is an essential property for systems enduring control dependent perturbations, since process-irrelevant correction could destabilize the system [40]. The approach of state dependent Riccati equations was first adopted by Pearson in 1960s and the notion was duly modified as well as applied on real time set-ups by Beeler upon introducing control non-linearity [41, 42]. This approach was effective for solving Hamilton-Jacobi-Bellman equation as a closed loop non-linear optimal control method. Nekoo et al. [43] utilized this perception and demonstrated the adaptability of a modified state-dependent Riccati equation approach for a highly coupled non-linear system with complex weight matrix. But still the associated models do not incorporate any spatial dependence of the controlling state.

Till date the application of Riccati-based approach is mostly restricted to lumped parameter models. For such systems, standard practice for closed-loop, real-time, control heavily relies on an explicit expression relating the current operating state of the system to the stipulated control variable [43]; the linearized control command is therefore simply expressed in terms of a gain matrix applied to the existing system state. The gain matrix is determined as the solution of a time-dependent Riccati equation, particularly when the control command is derived over an infinite horizon. In that case, an efficient closed-loop optimal control 
becomes more promising when coupled with a state estimator (for instance, Kalman filter based state estimator), which equips the controller with an estimate of the actual prevailing state of the system. This approach has become very trendy as it bridges the gap between existing state/policy and its desired optimal counterparts [43, 44]. Typical studies involving a Riccati equation-based approach deal with the control of induced perturbations affecting a linearized model such as boundary layer transition control or instability development control. Additionally, gain-scheduling could be an alternate choice to achieve high-performance (closed-loop) global control without heavy computations where the notion of "envelopes" is introduced in the state space of the system followed by the derivation of control specific to each envelope; these envelopes generally incorporate time dependent optimal estimations in terms of system state or Riccati variables. Numerous aeronautical applications used to rely on such a control scheme which eventually mandated several controllers to be resolved and transition between the controllers is quite challenging [43, 44].

\subsection{Novelty of the Thesis}

The novelty (significance) of this thesis is primarily associated with the application of Riccati transformation to a detailed non-linear, autonomous, unsteady state, distributed parameter mathematical model. Simultaneously, state-dependent linearized control law was formulated by strategically allocating boundary conditions to the perturbed expression of stationary condition. The following steps were performed in this study:

1. We develop the theoretical structure of closed loop optimal control (described in Chapter 3 by perturbing open loop optimal control framework (explained in Chapter 2); thus, we formulate feedback optimal control necessary conditions in terms Riccati equations and proportional gain control law.

2. We apply open loop optimal control strategy (Chapter 2) to a distributed parameter process model (Section 4.2.1) and obtain a repository in terms of optimal policy 
and other process variables (optimal state and optimal gain) at known process inputs (Section 4.4.1).

3. We perturb real-time optimal trajectory; we deploy the stored repository to interpolate the closest gain and apply necessary control adjustment to steer the process to optimality at new operating condition (described in Section 4.4.2).

4. Finally, we analyze various results and endeavour to scrutinize the effectiveness of the developed closed loop control strategy (Section 4.4.2).

Most important feature of this state-feedback control strategy is: the real time simulation is entirely based on the interpolation with respect to the stored optimal repository that reduces computational overhead as we do not need to calculate the optimal control problem every time at tweaked system state. Therefore, chances of process or economic loss can be effectively diminished.

This Feedback control algorithm can be applied to any physical system. We endeavored to examine the effectiveness of this closed loop optimal control strategy for a laboratory scale heavy oil reservoir (described in Section 4.1) in presence of pressure upset and we used $T_{\text {int }}(t)$ as the control function. 


\section{Chapter 2}

\section{Optimal Control Background}

In this chapter, we engage with the theoretical background of open loop optimal control problem. We will define and analyze the problem, then we will discuss the solution strategy and finally, we will conclude with its limitations in terms of real time application.

\subsection{Problem Statement}

The objective of an optimal control problem is to find a continuous control function $\mathbf{u}(t)$ that necessarily optimizes a pre-defined objective functional encompassing an autonomous integrand $F[45]$ as follows:

$$
I=\int_{0}^{t_{\mathrm{f}}} F[\mathbf{y}(t), \mathbf{u}(t)] \mathrm{d} t ; \quad \mathbf{y} \in \mathbb{R}^{n}, \mathbf{u} \in \mathbb{R}^{m}
$$

where $\mathbf{y}(t)$ and $\mathbf{u}(t)$ are state and control vectors

$$
\boldsymbol{y}=\left[\begin{array}{llll}
\boldsymbol{y}_{0} & \boldsymbol{y}_{1} & \ldots & \boldsymbol{y}_{n-1}
\end{array}\right]^{\top} \text { and } \boldsymbol{u}=\left[\begin{array}{lllll}
\boldsymbol{u}_{0} & \boldsymbol{u}_{1} & \ldots & \boldsymbol{u}_{n-1}
\end{array}\right]^{\top}
$$

subjected to the constraint differential equations

$$
-\frac{\mathrm{d} \mathbf{y}}{\mathrm{d} t}+\mathbf{f}[\mathbf{y}(\mathbf{t}), \mathbf{u}(\mathbf{t})] \equiv \mathbf{G}=\mathbf{0} ;
$$

where $\mathbf{f}$ is the autonomous function vector

$$
\boldsymbol{f}=\left[\begin{array}{llll}
\boldsymbol{f}_{0} & \boldsymbol{f}_{1} & \ldots & \boldsymbol{f}_{n-1}
\end{array}\right]^{\top}
$$


along with the initial conditions

$$
\mathbf{y}(0)=\mathbf{y}_{0} .
$$

Differential equations 2.2 is alternatively called the system of state equations since they describe states of the system $[\mathbf{y}(t)]$ as a function of the independent variable $t$. Both $F$ and f may have continuous partial derivatives with respect to both state and control vector. In this case, the final time $\left(t_{\mathrm{f}}\right)$ is specified and the overall analysis is performed in the interval $0 \leq t \leq t_{\mathrm{f}}$ [46, 47, 48].

\subsection{Augmented Functional}

In case $\mathbf{u}(t)$ has indirect non-linear influence on the integrand $F$ or $\mathbf{f}$ is highly non-linear [in terms of $\mathbf{y}(t)$ or $\mathbf{u}(t)$ ], we can not find any explicit solution for the control dependent state i.e., $\mathbf{y}=\mathbf{y}(t, \mathbf{u})$. To obtain the solution of the optimization problem, we adjoin the state equation constraints to $I$ using undetermined Lagrange multiplier vector $\boldsymbol{\lambda}(t)$ or co-state variables [46] and obtain the augmented functional,

$$
J=\int_{0}^{t_{\mathrm{f}}}\left(F+\boldsymbol{\lambda}^{\top} \mathbf{G}\right) \mathrm{d} t
$$

where $\boldsymbol{\lambda}$ is the lagrange multiplier vector

$$
\boldsymbol{\lambda}=\left[\begin{array}{llll}
\lambda_{0} & \lambda_{1} & \ldots & \lambda_{n-1}
\end{array}\right]^{\top} .
$$

For the given initial condition, optimization of $J$ is equivalent to the optimization of $I$ constrained by the state equations because the augmented functional consists of all terms of both the objective functional and the state equation constraints. Furthermore, we apply principles of variational calculus in order to analyze the problem. The analysis necessarily leads to the solution of the optimal control problem. 


\subsection{Analysis of Optimal Control}

At the optimum of $J, \delta J=0$ [47]. Hence,

$$
\delta J=\int_{0}^{t_{\mathrm{f}}}\left[\delta F+\delta \boldsymbol{\lambda}^{\top} \cdot \mathbf{G}+\boldsymbol{\lambda}^{\top} \delta \mathbf{G}\right] \mathrm{d} t .
$$

The result is necessarily subjected to the following pre-conditions:

1. The derivatives of $F$ and $f$ with respect to $\mathbf{y}$ and $\mathbf{u}$ are continuous in the vicinity of the optimum; and

2. There exists at least one set of variations at each $t$ in $\left(0, t_{\mathrm{f}}\right]$ for which the variation of $\mathbf{G}$ is not zero at the optimum [47].

In the context of equation 2.4 .

$$
\delta F=F_{\mathbf{y}} \delta \mathbf{y}+F_{\mathbf{u}} \delta \mathbf{u},
$$

and

$$
\delta \mathbf{G}=\mathbf{G}_{\mathbf{y}} \delta \mathbf{y}+\mathbf{G}_{\mathbf{u}} \delta \mathbf{u}+\mathbf{G}_{\dot{\mathbf{y}}} \delta \dot{\mathbf{y}} .
$$

Eventually, $\delta J$ depends upon arbitrary functions $\delta \mathbf{y}, \delta \mathbf{u}$ and $\delta \boldsymbol{\lambda}$. But, $\delta \dot{\mathbf{y}}$ is not arbitrary and depends on $\delta \mathbf{y}$. Hence by applying integration by parts with respect to $t$, we obtain

$$
\int_{0}^{t_{\mathrm{f}}} \boldsymbol{\lambda}^{\top} \mathbf{G}_{\dot{\mathbf{y}}} \delta \dot{\mathbf{y}} \mathrm{d} t=\left[\boldsymbol{\lambda}^{\top} \mathbf{G}_{\dot{\mathbf{y}}} \delta \mathbf{y}\right]_{t=t_{\mathrm{f}}}-\left[\boldsymbol{\lambda}^{\top} \mathbf{G}_{\dot{\mathbf{y}}} \delta \mathbf{y}\right]_{t=0}+\int_{0}^{t_{\mathrm{f}}}\left[\frac{\mathrm{d}\left(\boldsymbol{\lambda}^{\top} \mathbf{G}_{\dot{\mathbf{y}}}\right)}{\mathrm{d} t}\right] \delta \mathbf{y} \mathrm{d} t .
$$

Combining equations 2.5, 2.6 and 2.7 with equation 2.4, we obtain

$$
\begin{aligned}
\delta J= & {\left[\boldsymbol{\lambda}^{\top} \mathbf{G}_{\dot{\mathbf{y}}} \delta \mathbf{y}\right]_{t=t_{\mathrm{f}}}-\left[\boldsymbol{\lambda}^{\top} \mathbf{G}_{\dot{\mathbf{y}}} \delta \mathbf{y}\right]_{t=0}+\int_{0}^{t_{\mathrm{f}}}\left[F_{\mathbf{y}}+\boldsymbol{\lambda}^{\top} \mathbf{G}_{\mathbf{y}}+\frac{\mathrm{d}\left(\boldsymbol{\lambda}^{\top} \mathbf{G}_{\dot{\mathbf{y}}}\right)}{\mathrm{d} t}\right] \delta \mathbf{y} \mathrm{d} t } \\
& +\int_{0}^{t_{\mathrm{f}}}\left[F_{\mathbf{u}}+\boldsymbol{\lambda}^{\top} \mathbf{G}_{\mathbf{u}}\right] \delta \mathbf{u} \mathrm{d} t+\int_{0}^{t_{\mathrm{f}}} \delta \boldsymbol{\lambda}^{\top} \cdot \mathbf{G} \mathrm{d} t .
\end{aligned}
$$


Initially, system state is specified at $\mathbf{y}_{0}$. Thus at $t=0, \delta \mathbf{y}=\mathbf{0}$. At the final time, we could specify the Lagrange multipliers at zero value i.e., $\boldsymbol{\lambda}_{t_{\mathrm{f}}}=\mathbf{0}$ [4]. Thus Equation 2.8 takes the following form:

$$
\delta J=\int_{0}^{t_{\mathrm{f}}} \underbrace{\left[F_{\mathbf{y}}+\boldsymbol{\lambda}^{\top} \mathbf{G}_{\mathbf{y}}+\frac{\mathrm{d}\left(\boldsymbol{\lambda}^{\top} \mathbf{G}_{\dot{\mathbf{y}}}\right)}{\mathrm{d} t}\right]}_{=\delta J_{1}} \delta \mathbf{y} \mathrm{d} t+\int_{0}^{t_{\mathrm{f}}} \underbrace{\left[F_{\mathbf{u}}+\boldsymbol{\lambda}^{\top} \mathbf{G}_{\mathbf{u}}\right]}_{=\delta J_{2}} \delta \mathbf{u} \mathrm{d} t+\int_{0}^{t_{\mathrm{f}}} \delta \boldsymbol{\lambda}^{\top} \underbrace{[\mathbf{G}]}_{=\delta J_{3}} \mathrm{~d} t .
$$

Since $\delta \mathbf{y}, \delta \mathbf{u}$ and $\delta \boldsymbol{\lambda}$ are arbitrary in the above equation, $\delta J_{1}=0, \delta J_{2}=0$ and $\delta J_{3}=0$ must be satisfied in order to ensure $\delta J=0$ [47, 48]. By necessity, we obtain

$$
\begin{aligned}
& F_{\mathbf{y}}+\boldsymbol{\lambda}^{\top} \mathbf{G}_{\mathbf{y}}+\frac{\mathrm{d}\left(\boldsymbol{\lambda}^{\top} \mathbf{G}_{\dot{\mathbf{y}}}\right)}{\mathrm{d} t}=\mathbf{0}, \\
& F_{\mathbf{u}}+\boldsymbol{\lambda}^{\top} \mathbf{G}_{\mathbf{u}}=\mathbf{0}, \text { and } \\
& \mathbf{G}=\mathbf{0}
\end{aligned}
$$

\subsection{Hamiltonian Based Approach}

If we assume that system states $\mathbf{y}$ can be represented by the fully discretized linear differential equations

$$
\frac{\mathrm{d} \mathbf{y}}{\mathrm{d} t}=\mathbf{A y}+\mathbf{B u} \equiv \mathbf{f}
$$

subjected to the initial conditions

$$
\mathbf{y}(0)=\mathbf{y}_{0},
$$

then the fully discretized objective functional $I$ becomes linear quadratic as the following

$$
I=\int_{0}^{t_{\mathrm{f}}}\left(\mathbf{y}^{\top} \mathbf{C y}+\mathbf{u}^{\top} \mathbf{D} \mathbf{u}\right) \mathrm{d} t \equiv \int_{0}^{t_{\mathrm{f}}} F[\mathbf{y}(t), \mathbf{u}(t)] \mathrm{d} t .
$$


This approach enables us to introduce a mnemonic function called the Hamiltonian (by combining 2.13 and 2.14 with the aid of undetermined $\boldsymbol{\lambda}$, [47]) given by

$$
H=\boldsymbol{\lambda}^{\top} \mathbf{f}+F .
$$

From the definition of the Hamiltonian, it is convenient to verify and compare that

$$
\begin{aligned}
& H_{\boldsymbol{\lambda}}=\frac{\partial H}{\partial \boldsymbol{\lambda}}=\dot{\mathbf{y}} \equiv \underbrace{-\frac{\mathrm{d} \mathbf{y}}{\mathrm{d} t}+\mathbf{f}[\mathbf{y}(\mathbf{t}), \mathbf{u}(\mathbf{t})]=\mathbf{0}}_{\text {state equations }}, \\
& H_{\mathbf{y}}=\frac{\partial H}{\partial \mathbf{y}}=-\dot{\boldsymbol{\lambda}}^{\top} \equiv \underbrace{F_{\mathbf{y}}+\boldsymbol{\lambda}^{\top} \mathbf{G}_{\mathbf{y}}+\frac{\mathrm{d}\left(\boldsymbol{\lambda}^{\top} \mathbf{G}_{\dot{\mathbf{y}}}\right)}{\mathrm{d} t}=\mathbf{0}}_{\text {co-state equations }},
\end{aligned}
$$

and

$$
H_{\mathbf{u}}=\frac{\partial H}{\partial \mathbf{u}}=0 \equiv \underbrace{F_{\mathbf{u}}+\boldsymbol{\lambda}^{\top} \mathbf{G}_{\mathbf{u}}=\mathbf{0}}_{\text {stationarity condition }} .
$$

\subsection{Pontryagin's Minimum Principle}

We deploy Pontryagin's minimum principle to derive necessary conditions for the minimum of the above optimal control problem [47]. According to this principle, for any admissible choice of control $\mathbf{u}=\hat{\mathbf{u}}+\delta \mathbf{u}, H(\hat{\mathbf{y}}, \hat{\boldsymbol{\lambda}}, \hat{\mathbf{u}})$ is minimum at any instant of time. Thus,

$$
H(\hat{\mathbf{y}}, \hat{\boldsymbol{\lambda}}, \hat{\mathbf{u}}) \leq H(\hat{\mathbf{y}}, \hat{\boldsymbol{\lambda}}, \mathbf{u}), \quad 0 \leq t \leq t_{\mathrm{f}} .
$$

With the Hamiltonian based approach, Equation 2.9 yields the following:

$$
\delta J=\int_{0}^{t_{\mathrm{f}}} \underbrace{\left[H_{\mathbf{y}}+\dot{\boldsymbol{\lambda}}^{\top}\right]}_{=\delta J_{1}} \delta \mathbf{y} \mathrm{d} t+\int_{0}^{t_{\mathrm{f}}} \underbrace{\left[H_{\mathbf{u}}\right]}_{=\delta J_{2}} \delta \mathbf{u} \mathrm{d} t+\int_{0}^{t_{\mathrm{f}}} \underbrace{\left[-\dot{\mathbf{y}}+H_{\boldsymbol{\lambda}}\right]}_{=\delta J_{3}} \delta \boldsymbol{\lambda} \mathrm{d} t .
$$

For a given optimal control $\hat{\mathbf{u}}$, if both state and co-state equations are satisfied (for predetermined, fixed initial and terminal conditions respectively), we are eventually left with 
the stationarity condition. For sufficiently small $\delta \mathbf{u}$, we can apply first order Taylor expansion on the Hamiltonian $H_{\mathbf{u}}$ at $(\hat{\mathbf{y}}, \hat{\boldsymbol{\lambda}}, \hat{\mathbf{u}}+\delta \mathbf{u})$ and we obtain the following:

$$
\begin{aligned}
\delta J= & J(\hat{\mathbf{y}}, \hat{\boldsymbol{\lambda}}, \hat{\mathbf{u}}+\delta \mathbf{u})-J(\hat{\mathbf{y}}, \hat{\boldsymbol{\lambda}}, \hat{\mathbf{u}})=I(\hat{\mathbf{y}}, \hat{\mathbf{u}}+\delta \mathbf{u})-I(\hat{\mathbf{y}}, \hat{\mathbf{u}}) \\
& =\int_{0}^{t_{f}}\left[H_{\mathbf{u}}(\hat{\mathbf{y}}, \hat{\boldsymbol{\lambda}}, \hat{\mathbf{u}}+\delta \mathbf{u})-H_{\mathbf{u}}(\hat{\mathbf{y}}, \hat{\boldsymbol{\lambda}}, \hat{\mathbf{u}})\right] \delta \mathbf{u} \mathrm{d} t \\
& =\int_{0}^{t_{f}}\left[H_{\mathbf{u}}(\hat{\mathbf{y}}, \hat{\boldsymbol{\lambda}}, \mathbf{u})-H_{\mathbf{u}}(\hat{\mathbf{y}}, \hat{\boldsymbol{\lambda}}, \hat{\mathbf{u}})\right] \delta \mathbf{u} \mathrm{d} t
\end{aligned}
$$

where $\mathbf{u}$ is the perturbed control. Depending on $\mathbf{u}$, we can have five different cases [47]:

1. $\mathbf{u}$ has no constraints: In this case, $\delta \mathbf{u}$ can have any value and a non-zero $H(\hat{\mathbf{y}}, \hat{\boldsymbol{\lambda}}, \hat{\mathbf{u}})$ leads to $\delta I<0$. Hence, $H(\hat{\mathbf{y}}, \hat{\boldsymbol{\lambda}}, \hat{\mathbf{u}})$ must be zero for the minimum of $\delta I$ and it leads to $H(\hat{\mathbf{y}}, \hat{\boldsymbol{\lambda}}, \hat{\mathbf{u}})=H(\hat{\mathbf{y}}, \hat{\boldsymbol{\lambda}}, \mathbf{u})$.

2. $\mathbf{u}_{\min }<\mathbf{u}<\mathbf{u}_{\max }$ : Provides $H(\hat{\mathbf{y}}, \hat{\boldsymbol{\lambda}}, \hat{\mathbf{u}})=H(\hat{\mathbf{y}}, \hat{\boldsymbol{\lambda}}, \mathbf{u})$ for the same reason as stated above.

3. $\mathbf{u} \leq \mathbf{u}_{\max }$ : Provides $H(\hat{\mathbf{y}}, \hat{\boldsymbol{\lambda}}, \hat{\mathbf{u}}) \leq H(\hat{\mathbf{y}}, \hat{\boldsymbol{\lambda}}, \mathbf{u})$ because $\delta \mathbf{u}$ can take only negative values and in order to ensure $I(\hat{\mathbf{y}}, \mathbf{u})-I(\hat{\mathbf{y}}, \hat{\mathbf{u}}) \geq 0$.

4. $\mathbf{u} \geq \mathbf{u}_{\text {min }}$ : Provides $H(\hat{\mathbf{y}}, \hat{\boldsymbol{\lambda}}, \hat{\mathbf{u}}) \leq H(\hat{\mathbf{y}}, \hat{\boldsymbol{\lambda}}, \mathbf{u})$ because $\delta \mathbf{u}$ can take only positive values and in order to ensure $I(\hat{\mathbf{y}}, \mathbf{u})-I(\hat{\mathbf{y}}, \hat{\mathbf{u}}) \geq 0$.

5. $F=F(|\mathbf{u}|)$ : When $\mathbf{u}=0$, we can not apply the stationarity condition. But $H(\hat{\mathbf{y}}, \hat{\boldsymbol{\lambda}}, \hat{\mathbf{u}}) \leq$ $H(\hat{\mathbf{y}}, \hat{\boldsymbol{\lambda}}, \mathbf{u})$ provides the necessary condition for the minimum of $I$.

The above cases show that Pontryagin's minimum principle provides necessary condition for the minimum of $\delta J$ [47]. 


\subsection{Summary of Necessary Conditions}

From the analysis of the optimal control problem, we obtain the necessary conditions (valid in the interval of $0 \leq t \leq t_{\mathrm{f}}$ ) as follows:

The system of state equations:

$$
-\frac{\mathrm{d} \mathbf{y}}{\mathrm{d} t}+\mathbf{f}[\mathbf{y}(\mathbf{t}), \mathbf{u}(\mathbf{t})]=\mathbf{0}, \text { with the initial condition } \mathbf{y}(0)=\mathbf{y}_{\mathbf{0}},
$$

the system of co-state equations:

$$
F_{\mathbf{y}}+\boldsymbol{\lambda}^{\top} \mathbf{G}_{\mathbf{y}}+\frac{\mathrm{d}\left(\boldsymbol{\lambda}^{\top} \mathbf{G}_{\dot{\mathbf{y}}}\right)}{\mathrm{d} t}=\mathbf{0}, \text { with the final condition } \boldsymbol{\lambda}\left(t_{\mathrm{f}}\right)=\mathbf{0},
$$

and the stationarity condition:

$$
F_{\mathbf{u}}+\boldsymbol{\lambda}^{\top} \mathbf{G}_{\mathbf{u}}=\mathbf{0}
$$

\subsection{Solution of Optimal Control Problem}

The solution of optimal control problem requires satisfaction of differential equations subject to initial as well as final conditions. In this section, we discuss about the gradient method, which is quite effective for solving a wide range of optimal control problems [47]. In this method, the state and co-state equations are solved using initial guesses for the control $\mathbf{u}$ and the final time $t_{\mathrm{f}}$. The guessed $\mathbf{u}$ is then improved using the stationarity condition $H_{\mathbf{u}}$ in the interval $0 \leq t \leq t_{\mathrm{f}}$. The stepwise iterative procedure of the gradient method is:

1. We guess for the initial control function $\mathbf{u}$.

2. We integrate the state equations forward to the final time using the initial conditions and the control function.

3. We calculate the objective functional using the control functions and the corresponding state and co-state. 
4. Thus, we integrate the co-state equations backward to initial time using the final conditions at the final time, the control functions, and the state determined in step 3.

5. Finally, we improve the control function using the gradient information (until the stationarity condition approaches zero) and repeat Step 2 onward until there is no further reduction in the objective functional.

For sufficiently small $\epsilon, \delta \mathbf{u}=-\epsilon H_{\mathbf{u}}$; and the changes in $\delta \mathbf{u}$ is small enough so that the change in $J$ is given by $J(\mathbf{u}+\delta \mathbf{u})-J(\mathbf{u})=\delta J$. This change in $J$ to be minimized. Hence, based on this strategy, most improved functional value $J_{\text {next }}$ results from $\mathbf{u}_{\text {next }}(t)=\mathbf{u}(t)+\delta \mathbf{u}(t)$ and the corresponding state $\mathbf{y}_{\text {next }}$ [47]. Optimal control theory is applied in Section 4.4.1.

\section{Challenges in Optimal Operation}

The basic challenge associated with the real time application of open loop optimal control strategy is computational overhead. For deterministic dynamics, open-loop control algorithm generates a sequence of optimal state signals experiencing zero perturbations; in other words, open-loop policies do not have direct control regulation on the processes performing at its optimality. Presence of disturbances in the system state (pressure in our case, Chapter 4) often leads to sub-optimal or non-optimal behaviour, and this phenomenon can be effectively addressed by computing error feedback in optimization calculations.

A feed-forward scheme could also mitigate this setback but it mandates distinguished sensor and model for each of the disturbances; on contrary, a feedback strategy imparts control action, based on a unique process model, only when the output gets deviated from the set point (described in Chapter 3). Control action, based on this error, effectively bridges the gap between the real time perturbed state and the desired optimal state. Moreover, solving the entire optimal control problem at tweaked boundary condition is not a convincing approach as the iterative solution is quite time consuming or, computationally intensive. These perceptions invoke the idea of incorporation of an effective feedback control strategy (described in Section 3.5) in real time applications. 


\section{Chapter 3}

\section{Theoretical development}

In this chapter, we develop Riccati equations and state feedback control law based on the open loop optimal control necessary conditions discussed in Chapter 2. We introduce the Riccati based approach to formulate state feedback control strategy and apply the same to a distributed parameter system.

\subsection{Riccati Based Approach}

From the theoretical derivation based on the Hamiltonian approach (as described in Section 2.4 we obtain

$$
\begin{aligned}
& H_{\boldsymbol{\lambda}}=\frac{\partial H}{\partial \boldsymbol{\lambda}}=\dot{\mathbf{y}}=\mathbf{A y}+\mathbf{B u}, \\
& H_{\mathbf{y}}=\frac{\partial H}{\partial \mathbf{y}}=-\dot{\boldsymbol{\lambda}}^{\top}=\mathbf{C y}+\mathbf{A}^{\top} \boldsymbol{\lambda},
\end{aligned}
$$

and

$$
H_{\mathbf{u}}=\frac{\partial H}{\partial \mathbf{u}}=\mathbf{0}=\mathbf{D u}+\mathbf{B}^{\top} \boldsymbol{\lambda} .
$$

Thus we have from Equation 3.3

$$
\mathbf{u}=-\mathbf{D}^{-1} \mathbf{B}^{\top} \boldsymbol{\lambda} .
$$


Eventually, we obtain from Equation 3.1 and 3.4

$$
\dot{\mathbf{y}}=\mathbf{A y}-\mathrm{BD}^{-1} \mathbf{B}^{\top} \boldsymbol{\lambda},
$$

and from Equation 3.2

$$
\dot{\boldsymbol{\lambda}}=-\mathbf{C y}-\mathbf{A}^{\top} \boldsymbol{\lambda} .
$$

One possible solution of this problem is represented by the form

$$
\boldsymbol{\lambda}(t)=\mathbf{S}(t) \mathbf{y}(t)
$$

which is known as Riccati transformation [48, 49, 50]. Consequently, Equations 3.5 and 3.6 yield

$$
\begin{aligned}
& \dot{\mathbf{y}}=\mathrm{Ay}-\mathrm{BD}^{-1} \mathbf{B}^{\top} \mathrm{Sy}, \text { and } \\
& \mathbf{S} \dot{\mathbf{y}}+\dot{\mathbf{S}} \mathbf{y}=-\mathbf{C y}-\mathbf{A}^{\top} \mathbf{S y}
\end{aligned}
$$

By substituting equation 3.8 into equation 3.9 , we obtain

$$
\dot{\mathbf{S}}=-\mathbf{S A}-\mathbf{A}^{\top} \mathbf{S}+\mathbf{S B D}^{-1} \mathbf{B}^{\top} \mathbf{S}-\mathbf{C} ; \text { known as Riccati equation, }
$$

with the linearized proportional gain feedback control law

$$
\mathbf{u}=-\mathbf{D}^{-1} \mathbf{B}^{\top} \boldsymbol{\lambda}=-\mathbf{K}(t) \mathbf{y}(t) \text {; where, } \mathbf{K} \text { is the proportional gain parameter. }
$$

In the context of the above analysis, if we change the initial conditions in terms of $\mathbf{y}\left(t_{0}\right)$, the open loop optimal control policy needs to be recalculated as we can not apply superposition principle to quickly adjust the deviations in initial condition and the non-linear dependence of the optimal control problem on the initial conditions [49]. Hence, in our case, final time $\left(t_{\mathrm{f}}\right)$ and initial conditions $\left(\mathbf{y}=\mathbf{y}_{0}\right)$ are pre-determined and fixed. When the process experiences an upset in any boundary condition, perturbation equations can be formulated 
which represent the system behaviour for fixed initial conditions in some domain close to the nominal state $\overline{\mathbf{y}}_{0}$, that is for $\left|\delta \mathbf{y}\left(t_{0}\right)\right| \leq \epsilon[48,50$. Thus, the closed loop optimal control problem can be formulated as the minimization of second variation of $J$ around a known optimal $(\delta J,[51])$

$$
\delta^{2} J=\int_{0}^{t_{\mathrm{f}}}\left[\delta \mathbf{u}^{T}\left(\frac{\partial^{2} H}{\partial \mathbf{u}^{2}}\right) \delta \mathbf{u}+\delta \mathbf{y}^{T}\left(\frac{\partial^{2} H}{\partial \mathbf{y} \partial \mathbf{u}}\right) \delta \mathbf{u}+\delta \mathbf{y}^{T}\left(\frac{\partial^{2} H}{\partial \mathbf{y}^{2}}\right) \delta \mathbf{y}\right] \mathrm{d} t
$$

subjected to the perturbed system of state equations (as the constraint)

$$
\frac{\mathrm{d}[\delta \mathbf{y}(t)]}{\mathrm{d} t}=\left(\frac{\partial \mathbf{f}}{\partial \mathbf{y}}\right) \delta \mathbf{y}+\left(\frac{\partial \mathbf{f}}{\partial \mathbf{u}}\right) \delta \mathbf{u}
$$

using $\delta \mathbf{u}$ or the adjustment to control as the control policy. The deviation in the system is represented by Equation 3.13 whose solution $\delta \mathbf{u}(t)$ and $\delta \mathbf{y}(t)$ represents optimal feedback corrections to the nominal open loop control [49]. But the solution of this optimal control problem at a perturbed boundary condition (or, system state) is computationally intensive or, time consuming (as described in section 2.7); eventually, chances of process or economic loss are more pronounced. Alternatively, we can deploy Riccati based approach and introduce the notion of Riccati transformation on the perturbed state $(\delta \mathbf{y})$ and adjustment to control $(\delta \mathbf{u})$ as

$$
\delta \boldsymbol{\lambda}(t)=\mathbf{S}(t) \delta \mathbf{y}(t)
$$

which is described in subsequent sections.

\subsection{Basis of Closed Loop Control}

Let us consider the open-loop problem of finding the control $\mathbf{u}(t)$ at the optimum of the objective functional

$$
I=\int_{0}^{t_{\mathrm{f}}} \int_{0}^{x_{0 \mathrm{f}}} \int_{0}^{x_{1 \mathrm{f}}} \int_{0}^{x_{2 \mathrm{f}}} F[\mathbf{y}(t), \mathbf{u}(t)] \mathrm{d} x_{2} \mathrm{~d} x_{1} \mathrm{~d} x_{0} \mathrm{~d} t ; \quad \mathbf{y} \in \mathbb{R}^{n}, \mathbf{x} \in \mathbb{R}^{3}, \mathbf{u} \in \mathbb{R}^{m}
$$


subject to the process model, or state equation

$$
\frac{\partial \mathbf{y}}{\partial t}=\mathbf{f} ; \quad \mathbf{y}(0)=\mathbf{y}_{0}, \quad \mathbf{p}=\mathbf{0}
$$

where $\mathbf{f}$ depends on $\mathbf{u}, \mathbf{y}$ and its spatial derivatives; and $\mathbf{p}$ is a vector of functions depending on $\mathbf{y}$ at spatial boundaries, $\mathbf{y}_{\partial}$, and its derivatives. This problem is equivalent to finding the optimum of the augmented functional,

$$
J=\int_{0}^{t_{\mathrm{f}}} \int_{0}^{x_{0 \mathrm{f}}} \int_{0}^{x_{1 \mathrm{f}}} \int_{0}^{x_{2 \mathrm{f}}}\left[F+\boldsymbol{\lambda}^{\top}(-\dot{\mathbf{y}}+\mathbf{f})\right] \mathrm{d} x_{2} \mathrm{~d} x_{1} \mathrm{~d} x_{0} \mathrm{~d} t
$$

where $\boldsymbol{\lambda}$ is the co-state variable vector. At the optimum of $J$, its variation $\delta J=0$. Using the principles of variational calculus, this result eventually provides the necessary conditionsdifferential co-state equation with zero co-states at the final time $t_{\mathrm{f}}$ along with boundary conditions, and the variational derivative of $J$ with respect to $\mathbf{u}$ being zero - which must be satisfied to obtain the optimal control policy $\hat{\mathbf{u}}(t)$ and the corresponding optimal state $\hat{\mathbf{y}}(t)$.

\subsection{Optimal Process under Upset}

For a process running with the optimal control policy, a process upset (i.e., a change in a parameter value) renders the state non-optimal. Given a sufficiently small upset, the change in $J$ from the optimal state $(\hat{\mathbf{y}}, \hat{\mathbf{u}})$ can be expressed as

$$
\Delta J=J(\mathbf{y}, \mathbf{u})-J(\hat{\mathbf{y}}, \hat{\mathbf{u}})=\underbrace{\delta J(\hat{\mathbf{y}}, \hat{\mathbf{u}})}_{=0}+\delta^{2} J(\hat{\mathbf{y}}, \hat{\mathbf{u}} ; \delta \mathbf{y}, \delta \mathbf{u})
$$

where $\delta \mathbf{y}=\mathbf{y}-\hat{\mathbf{y}}$, and $\delta \mathbf{u}=\mathbf{u}-\hat{\mathbf{u}}$. Thus, the restoration of the process to optimality under the upset can be posed as the optimal control problem of finding the control adjustment $\delta \mathbf{u}$ that minimizes $\delta^{2} J$ (i.e., the second variation of $J$ ) subject to the perturbed state governed by

$$
\frac{\partial(\delta \mathbf{y})}{\partial t}=\left.\frac{\partial \mathbf{f}}{\partial \mathbf{y}}\right|_{\hat{\mathbf{y}}, \hat{\mathbf{u}}} \delta \mathbf{y}+\left.\frac{\partial \mathbf{f}}{\partial \mathbf{u}}\right|_{\hat{\mathbf{y}}, \hat{\mathbf{u}}} \delta \mathbf{u} ; \quad \delta \mathbf{y}(0)=\delta \mathbf{y}_{\partial}=\mathbf{0}
$$




\subsection{Incorporation of State Feedback}

Discretizing the spatial domain using grid point spacing $\Delta x_{i}$ along each $x_{i}$-direction, we get

$$
\delta^{2} J(\hat{\mathbf{y}}, \hat{\mathbf{u}} ; \delta \mathbf{y}, \delta \mathbf{u})=\int_{0}^{t_{f}}\left(\left.\frac{1}{2} \delta \mathbf{u}^{\top} \frac{\partial^{2} H}{\partial \mathbf{u}^{2}}\right|_{\hat{\mathbf{y}}, \hat{\mathbf{u}}} \delta \mathbf{u}+\left.\delta \mathbf{y}^{\top} \frac{\partial^{2} H}{\partial \mathbf{y} \partial \mathbf{u}}\right|_{\hat{\mathbf{y}}, \hat{\mathbf{u}}} \delta \mathbf{u}+\left.\frac{1}{2} \delta \mathbf{y}^{\top} \frac{\partial^{2} H}{\partial \mathbf{y}^{2}}\right|_{\hat{\mathbf{y}}, \hat{\mathbf{u}}} \delta \mathbf{y}\right) \mathrm{d} t
$$

where

$$
H=\left(\boldsymbol{\lambda}^{\top} \mathbf{f}+\sum_{i=0}^{L-1} \sum_{j=0}^{M-1} \sum_{k=0}^{N-1} F_{i j k}\right) \Delta x_{0} \Delta x_{1} \Delta x_{2}
$$

with $\boldsymbol{\lambda}$ and $\mathbf{f}$ as vectors of their respective elements at all spatial grid points [51]. Thus, for $n$ state variables and $L, M$ and $N$ grid points along, respectively, $x_{0^{-}}, x_{1^{-}}$and $x_{2^{-}}$-directions,

$$
\begin{aligned}
\boldsymbol{\lambda}= & {\left[\begin{array}{lllll}
\lambda_{0} & \lambda_{1} & \ldots & \boldsymbol{\lambda}_{n-1}
\end{array}\right]^{\top} \text { and } } \\
\boldsymbol{\lambda}_{i}= & {\left[\begin{array}{llllllllll}
\lambda_{i, 0,0,0} & \lambda_{i, 0,0,1} & \ldots & \lambda_{i, 0,0, N-1} & \lambda_{i, 0,1,0} & \lambda_{i, 0,1,1} & \ldots & \lambda_{i, 0,1, N-1} & \ldots & \lambda_{i, 0, M-1,0} \\
\ldots & \lambda_{i, 0, M-1, N-1} & \ldots & \lambda_{i, L-1, M-1,0} & \lambda_{i, L-1, M-1,1} & \ldots & \lambda_{i, L-1, M-1, N-1}
\end{array}\right]^{\top} } \\
&
\end{aligned}
$$

Note that the minimization of $\delta^{2} J$ subject to Equation (3.18) with $\delta \mathbf{u}(t)$ as the control function is the linear quadratic regulator problem of optimal control with finite time horizon [51]. In the necessary condition for the minimum of $\delta^{2} J$, we introduce the Riccatti transformation,

$$
\delta \boldsymbol{\lambda}=\mathbf{S} \delta \mathbf{y}
$$

where $\delta \boldsymbol{\lambda}$ and $\delta \mathbf{y}$ have the same structure as of $\boldsymbol{\lambda}$ in Equations (3.21) and (3.22), and

$$
\mathbf{S}=\left[\begin{array}{cccc} 
& & & \\
\mathbf{S}_{0,0} & \mathbf{S}_{0,1} & \ldots & \mathbf{S}_{0, n-1} \\
\mathbf{S}_{1,0} & \mathbf{S}_{1,1} & \ldots & \mathbf{S}_{1, n-1} \\
\vdots & \vdots & \vdots & \vdots \\
\mathbf{S}_{n-1,0} & \mathbf{S}_{n-1,1} & \ldots & \mathbf{S}_{n-1, n-1}
\end{array}\right]
$$


is a block positive definite Riccati matrix, each element of which is a symmetric matrix of size $L M N \times L M N$. Finally, we incorporate into the necessary condition, the feedback control law

$$
\delta \mathbf{u}=-\mathbf{K} \delta \mathbf{y}
$$

where $\mathbf{K}$ is the gain matrix. With the above considerations, the necessary condition for the minimum of $\delta^{2} J$ results in the differential Riccati equation [48, 49, 50, 51]

$$
\frac{\mathrm{d} \mathbf{S}}{\mathrm{d} t}=-\mathbf{S A}-\mathbf{A}^{\top} \mathbf{S}+\mathbf{S B D}^{-1} \mathbf{B}^{\top} \mathbf{S}-\mathbf{C} ; \quad \mathbf{S}\left(t_{\mathrm{f}}\right)=\mathbf{S}_{\partial}=\mathbf{0}
$$

where

$$
\begin{aligned}
& \mathbf{A}=\left.\frac{\partial \mathbf{f}}{\partial \mathbf{y}}\right|_{\hat{\mathbf{y}}, \hat{\mathbf{u}}}, \quad \mathbf{B}=\left.\frac{\partial \mathbf{f}}{\partial \mathbf{u}}\right|_{\hat{\mathbf{y}}, \hat{\mathbf{u}}}, \quad \mathbf{C}=\left.\frac{\partial^{2} H}{\partial \mathbf{y}^{2}}\right|_{\hat{\mathbf{y}}, \hat{\mathbf{u}}}, \quad \mathbf{D}=\left.\frac{\partial^{2} H}{\partial \mathbf{u}^{2}}\right|_{\hat{\mathbf{y}}, \hat{\mathbf{u}}}, \\
& \text { and } \mathbf{E}=\left.\frac{\partial^{2} H}{\partial \mathbf{y} \partial \mathbf{u}}\right|_{\hat{\mathbf{y}}, \hat{\mathbf{u}}}
\end{aligned}
$$

and a stationarity condition for $\delta \mathbf{u}$ that provides the equation for the gain [51],

$$
\mathbf{K}=\mathbf{D}^{-1}\left(\mathbf{E}^{\top}+\mathbf{B}^{\top} \mathbf{S}\right)
$$

\subsection{Feedback Optimal Control Strategy}

Following is the strategy (Figure 3.1) to apply feedback control to a process susceptible to upsets in a parameter value:

\section{Offline Calculations}

For each, different value of a parameter expected to undergo upsets, determine and store a repository of the following:

1. the optimal state $\hat{\mathbf{y}}(t)$ and control $\hat{\mathbf{u}}(t)$ by solving the open-loop optimal control problem, and 
2. the gain $\mathbf{K}(t)$ by solving the differential Riccati equation and the gain equation

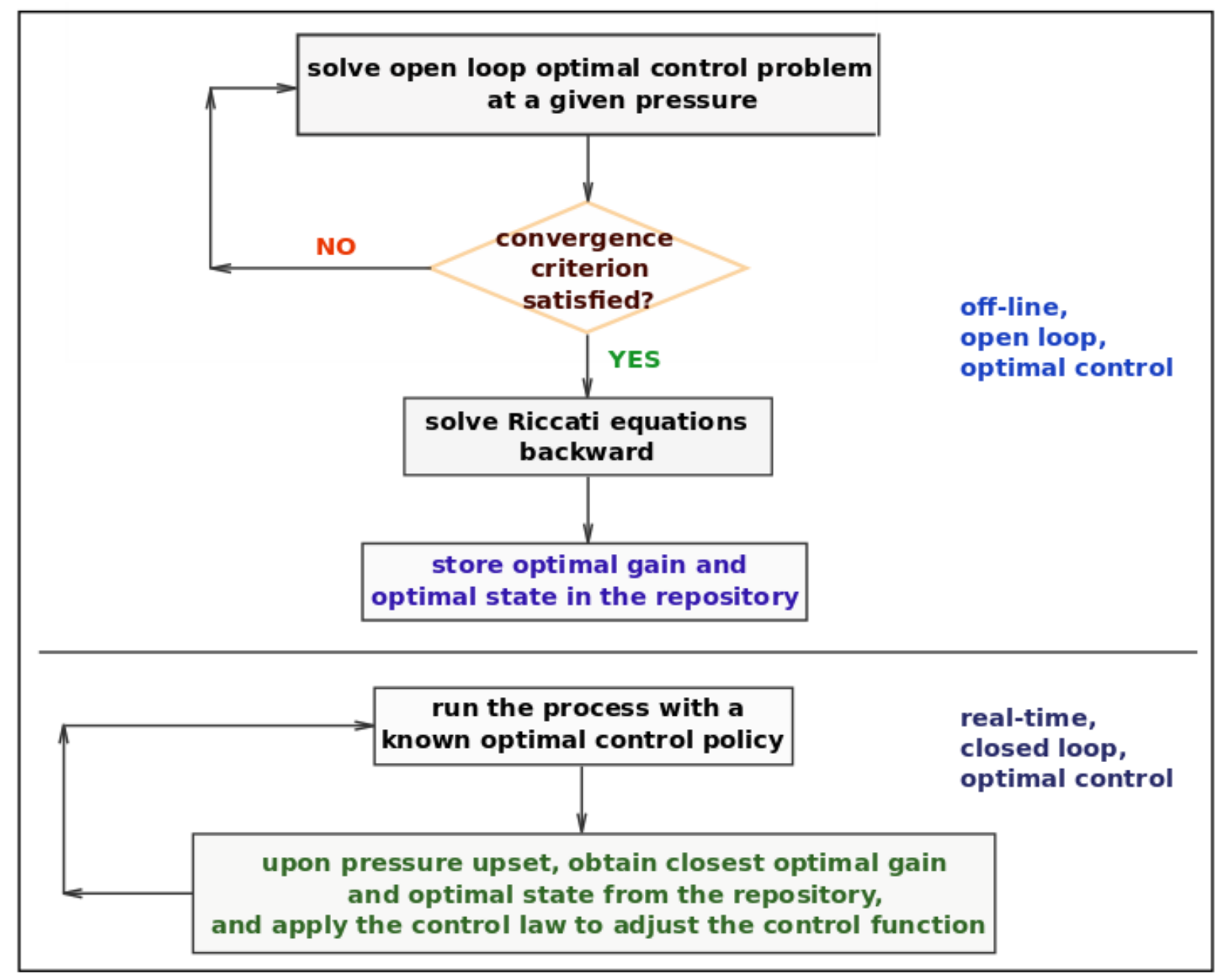

Figure 3.1: Closed Loop Optimal Control Strategy

\section{Real-Time Control}

Execute the process with a nominal value of the parameter, and pre-determined $\hat{\mathbf{u}}(t)$. Whenever there is an upset in the parameter, do the following:

1. obtain the interpolated $\hat{\mathbf{y}}(t)$ and $\mathbf{K}(t)$ from the repository at the current parameter value, and 
2. obtain and apply the improved control

$$
\hat{\mathbf{u}}(t)=\mathbf{u}(t)+\mathbf{K}(t)[\mathbf{y}(t)-\hat{\mathbf{y}}(t)]
$$

which is the control law given by Equation 3.25.

To summarize, we developed the theoretical background of a proportional gain statefeedback controller with time varying gain which will perform control adjustment so that the process gets steered to optimal process conditions and simultaneously minimize the variation in the objective functional [50].

\section{Advantage of Real Time Feedback Strategy}

This feedback control strategy is totally based on real time interpolation with respect to the stored optimal repository. Thus, the simulation or real time implementation mandates less CPU time as we do not need to re-calculate the optimal control problem all over again at tweaked boundary condition (or, system state); thus, chances of process or economic loss (mentioned in section 3.1) are effectively subdued. The solution strategy along with control algorithm and simulation results are described in section 4.4.2. 


\section{Chapter 4}

\section{Application}

We derive the necessary conditions of open-loop optimality (described in Chapter 2) and apply the feedback control strategy (explained in Chapter 3) to a distributed-parameter system of a labscale heavy oil reservoir to which a gas (nitrogen) is injected to produce heavy oil. In this chapter we discuss the following:

1. We discuss the experimental process.

2. We review model equations, necessary conditions of open loop optimal control and closed loop control framework.

3. We analyze different simulation results.

\subsection{Process Description}

In this section, the experimental procedure to determine the dependence of solubility $\left(w_{\text {int }}\right)$ of nitrogen on temperature $(T)$ and pressure $(P)$ is described. Details of experimental apparatus and various parts are described in Appendix $\mathrm{A}$.

\subsubsection{Experimental Set-up}

As shown in the schematic (Figure 4.1), nitrogen is injected to the pressure vessel containing a cylindrical mesh packed with heavy oil (setup equivalent to the geological core). 


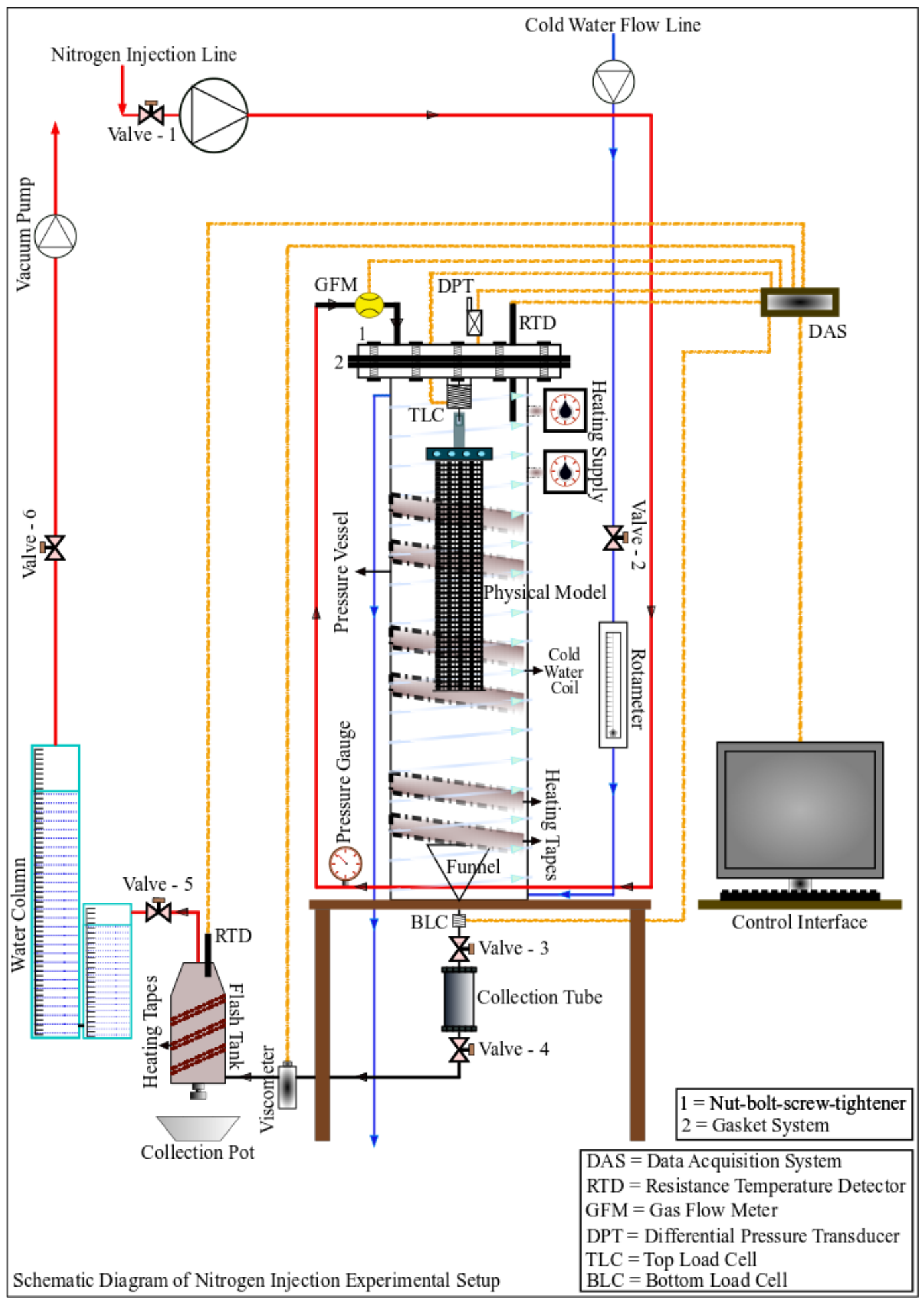

Figure 4.1: Schematic of Lab-scale Setup 
At the start of each experiment, air is purged out from the pressure vessel by applying 14.5 psi-absolute vacuum pressure through a vacuum pump. Then nitrogen gas is injected into the pressure vessel. The physical reservoir model is suspended from a load cell inside the vessel and it comes in contact with nitrogen. Elevated temperature aids in dissolution of nitrogen through oil. Eventually, viscosity of heavy oil drops and it starts flowing from the physical model; this live oil (nitrogen dissolved heavy oil) starts draining out from the bottom of the model by the effect of gravity. Thereafter, through a funnel, the live oil is accumulated inside the collection tube. The accumulated live oil is directed through the viscometer to the flash separation tank preheated at $70^{\circ} \mathrm{C}$. As the separated nitrogen from the flash tank enters water columns, the water level changes. This change reflects the amount of nitrogen dissolved in the oil. The flow regulating valve on the top is then vented to liberate nitrogen to the fume hood. The nitrogen-free dead oil from the flash tank is weighed. At the end of each experimental run, the gas flow is shut down and the pressure vessel is vented to atmosphere. Relative errors between the oil recoveries from the repeated experiments were found to be in the range of $0.8 \%$ to $1.3 \%$. Method of determination of interfacial concentration as a function of temperature and pressure is described in Appendix A, section A.3. Different experimental results are portrayed in Appendix D.

\subsection{Mathematical Equations}

The prime focus of the laboratory scale experimental investigation is to maximize the production of oil by injecting nitrogen through the porous media. The amount of accumulated dead oil is necessarily regulated by the cumulative effect of concentration, temperature and potential energy gradients. We couple these governing constraints with our objective functional and eventually derive necessary conditions for open loop optimality. Moreover, we formulate the Hamiltonian for the state feedback control scheme. In this section, we describe all these equations. In addition, we formulate different initial, terminal and boundary conditions corresponding to these equations. 


\subsubsection{Process Model}

We have three non-linear, autonomous, simultaneous process model equations in a distributed parameter system.

\section{Assumptions for Model Formulation}

Seven assumptions are made in order to simplify the system in terms of energy, mass and momentum balance [52, 53, 54]. These assumptions are:

1. The flow of the live oil through the porous medium along the vertical direction is governed by Darcy's law.

Justification: The flow of live oil is a creeping flow since Reynold's numbers (Re) calculated for the live oil flow are less than unity [54].

2. The diffusion of the nitrogen is pre-dominant along the radial direction only and diffusion in the vertical direction is neglected.

Justification: Diffusion occurs in the radial direction over a large surface area along the circumference of the cylindrical model. On the other hand, diffusion in the vertical direction occurs over a much smaller area starting at the top of the cylindrical model. Moreover, convection plays a dominant role in the vertical direction.

3. The porous medium possesses uniform porosity and permeability.

Justification: The oil is mixed uniformly with glass beads and packed in a cylindrical stainless steel wire mesh. Thus, the model has a uniform permeability, and no heterogeneities prevail.

4. The density of the live oil is assumed constant in the range of temperature variation (between $25^{\circ} \mathrm{C}$ to $90^{\circ} \mathrm{C}$ ).

Justification: A sensitivity analysis suffices that a small variation in live oil density [54] generates an insignificant change in the maximum objective functional. 
5. No chemical reaction takes place as the absorption of the solvent gas in heavy oil is purely a physical phenomenon [54].

6. Radioactive effects are neglected.

7. The specific heat capacity $\left(C_{\mathrm{p}}\right)$ and the thermal conductivity coefficient $(k)$ are assumed to be constant in the range of temperature variation.

Justification: A sensitivity analysis suffices that a small variation in specific heat and conductivity [54] generates an irrelevant change in the maximum objective functional.

Based on first principles, the process model is given by the following three state equations:

$$
\begin{aligned}
\frac{\partial w}{\partial t}= & \frac{D}{r} \frac{\partial w}{\partial r}+D \frac{\partial^{2} w}{\partial r^{2}}+\frac{\partial D}{\partial w}\left(\frac{\partial w}{\partial r}\right)^{2}+\frac{\partial D}{\partial T} \frac{\partial w}{\partial r} \frac{\partial T}{\partial r}+\frac{\alpha}{\phi \mu} \frac{\partial w}{\partial z}-\frac{\alpha w}{\phi \mu^{2}}\left(\frac{\partial \mu}{\partial w} \frac{\partial w}{\partial z}\right. \\
& \left.+\frac{\partial \mu}{\partial T} \frac{\partial T}{\partial z}\right), \\
\frac{\partial T}{\partial t}= & \frac{\gamma}{r} \frac{\partial T}{\partial r}+\gamma \frac{\partial^{2} T}{\partial r^{2}}+\gamma \frac{\partial^{2} T}{\partial z^{2}}+\frac{\alpha}{\phi \mu} \frac{\partial T}{\partial z}-\frac{\alpha T}{\phi \mu^{2}}\left(\frac{\partial \mu}{\partial w} \frac{\partial w}{\partial z}+\frac{\partial \mu}{\partial T} \frac{\partial T}{\partial z}\right), \\
\frac{\partial z}{\partial t}= & -\frac{\alpha}{\phi \bar{\mu}(r)}
\end{aligned}
$$

where $w$ is mass fraction of nitrogen in the reservoir, $T$ stands for temperature of the reservoir, and $z$ represents the height along the vertical direction or the moving boundary itself. Moreover, $\alpha=K_{\mathrm{r}} K \rho g$ where $K_{\mathrm{r}}$ and $K$ are, respectively, the relative permeability and permeability of the porous medium, $t$ is time, $r$ is the radial distance, $g$ is gravity, $\rho$ is oil density, $\phi$ is medium porosity with $\gamma=\frac{k}{\rho C_{\mathrm{p}}}$ where $C_{\mathrm{p}}$ as specific heat capacity and $k$ as thermal conductivity.

It may be noted that $D=D(w, P, T)$ is the dispersion coefficient of gas in heavy oil (refer to Appendix C.2), $\mu=\mu(w, P, T)$ is the heavy oil viscosity (see Appendix C.1), and $\bar{\mu}(r)$ is the average viscosity at $z=0$ as a function of $r$. Detailed derivation of these process models are explained in Appendix E and model simulation results (up to $163.85 \mathrm{~min}$ ) 
are described in Appendix F. The above equations are subject to the following initial and boundary conditions:

\section{Initial and Boundary Conditions}

Initially, height of bitumen is $Z_{0}(35 \mathrm{~cm})$ with no dissolved nitrogen. However, all surfaces have interfacial conditions in terms of both temperature and nitrogen at any point of time. Thus, initial conditions at $t=0$ are:

$$
\begin{aligned}
& w=\left\{\begin{array}{l}
0 \quad \forall 0<z<Z_{0}, 0<r<R \\
w_{\text {int }} \text { at } r=R \forall 0 \leq z \leq Z_{0} \text { and } z \in\left\{0, Z_{0}\right\} \forall 0 \leq r<R,
\end{array}\right. \\
& T= \begin{cases}T_{0} \quad \forall 0<z<Z_{0}, 0<r<R \\
T_{\text {int }} & \text { at } r=R \forall 0 \leq z \leq Z_{0} \text { and } z \in\left\{0, Z_{0}\right\} \forall 0 \leq r<R ; \text { and, }\end{cases} \\
& z=Z_{0} \quad \forall 0 \leq r \leq R .
\end{aligned}
$$

It is noteworthy that $r$ is the radius measured radially outward from the central vertical axis of the cylindrical physical model and $z$ is the vertical distance measured upward from the bottom of the cylindrical core as shown in the Figure 4.2. Also, initially $T_{0}, T_{\text {int }}=23{ }^{\circ} \mathrm{C}$. The boundary conditions at any point of time $t>0$ are

$$
\begin{aligned}
& w=w_{\text {int }} \text { at } r=R \forall 0 \leq z \leq Z_{0} \text { and } z \in\left\{0, Z_{0}\right\} \forall 0 \leq r<R, \\
& T=T_{\text {int }} \text { at } r=R \forall 0 \leq z \leq Z_{0} \text { and } z \in\left\{0, Z_{0}\right\} \forall 0 \leq r<R
\end{aligned}
$$

where $z(t, r)$ is the bitumen height inside porous media at any given $r$ and $t$. Additionally, due to symmetry,

$$
\frac{\partial T}{\partial r}, \frac{\partial w}{\partial r}=0 \quad \text { at } r=0 \forall 0 \leq z \leq Z_{0}
$$

at any time instant. Model equations with the associated initial and boundary conditions become constraints of the optimal control problem that is portrayed next. 


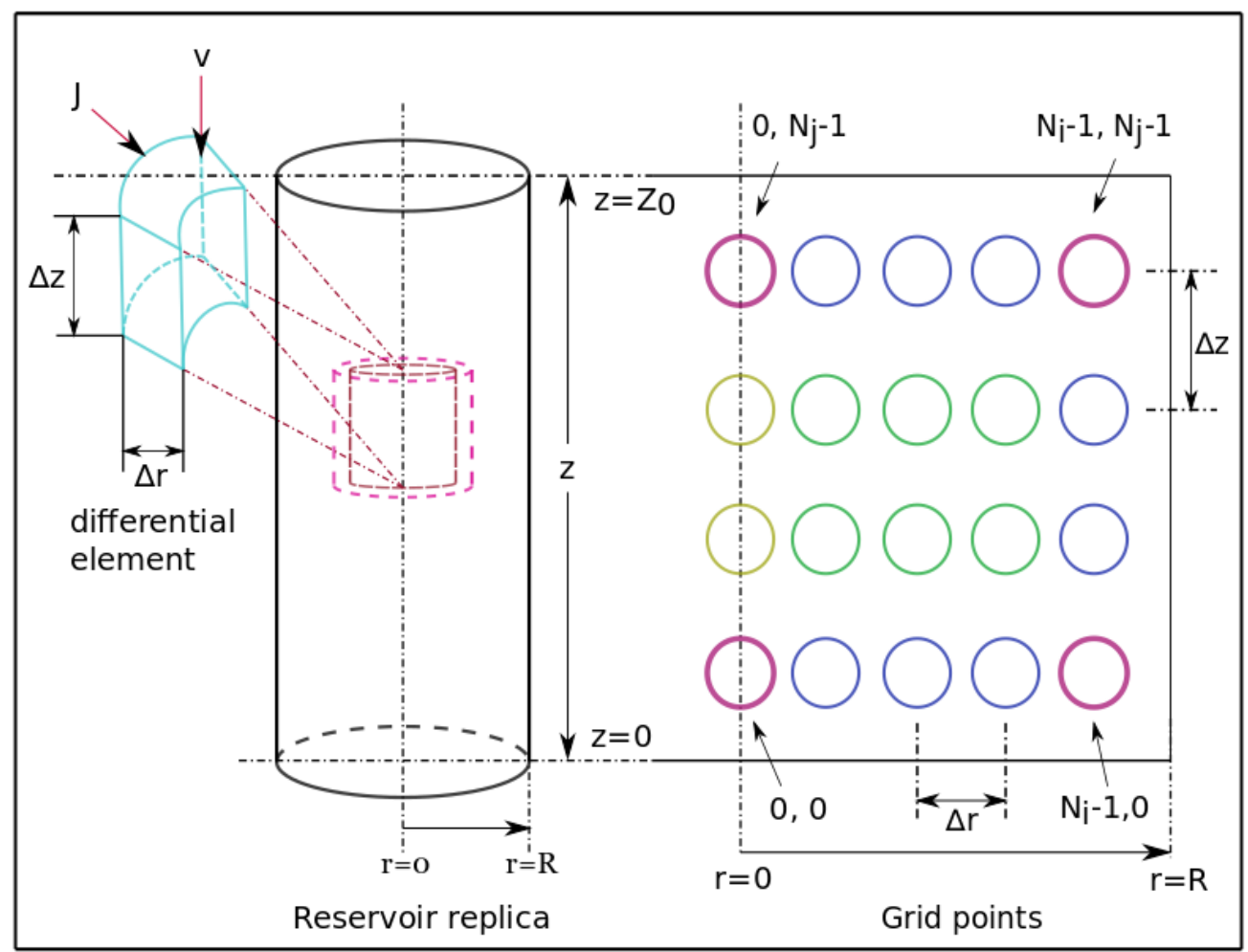

Figure 4.2: Cylindrical Geometry

\subsubsection{Objective Functional}

The mathematical model of the lab-scale nitrogen injection enhanced oil recovery process forms the basis for the optimal control problem; the objective of the same is to maximize oil production using interfacial temperature versus time as a control function.

\section{Objective Functional}

In the present case, the optimal control problem is to maximize heavy oil production given by the following objective functional

$$
I=\int_{0}^{t_{\mathrm{f}}} \dot{m} \mathrm{~d} t
$$

subjected to the satisfaction of the associated non-linear model equations or constraints. In the above expression, $t_{\mathrm{f}}$ is the final time (fixed and pre-determined) and $\dot{m}$ is the mass flow 
rate at any instant of time which is given by

$$
\dot{m}=\int_{0}^{R} 2 \pi \rho v_{0} r \mathrm{~d} r=\int_{0}^{R} \frac{2 \pi \rho \alpha r}{\bar{\mu}} \mathrm{d} r .
$$

Combining above two equations, we obtain

$$
I=\int_{0}^{t_{\mathrm{f}}} \int_{0}^{R}\left(\frac{2 \pi \rho \alpha r}{\bar{\mu}}\right) \mathrm{d} r \mathrm{~d} t=\int_{0}^{t_{\mathrm{f}}} \int_{0}^{R}\left(\frac{\beta r}{\bar{\mu}}\right) \mathrm{d} r \mathrm{~d} t
$$

where $\beta=2 \pi \rho \alpha$. The associated non-linear constraint equations are:

$$
-\frac{\partial T}{\partial t}+f_{1}=G_{1}(t, r, z)=0
$$

where

$$
\begin{aligned}
& f_{1}=\frac{\gamma}{r} \frac{\partial T}{\partial r}+\gamma \frac{\partial^{2} T}{\partial r^{2}}+\gamma \frac{\partial^{2} T}{\partial z^{2}}+\frac{\alpha}{\phi \mu} \frac{\partial T}{\partial z}-\frac{\alpha T}{\phi \mu^{2}}\left(\frac{\partial \mu}{\partial w} \frac{\partial w}{\partial z}+\frac{\partial \mu}{\partial T} \frac{\partial T}{\partial z}\right), \\
& -\frac{\partial w}{\partial t}+f_{2}=G_{2}(t, r, z)=0
\end{aligned}
$$

where

$$
f_{2}=\frac{D}{r} \frac{\partial w}{\partial r}+D \frac{\partial^{2} w}{\partial r^{2}}+\frac{\partial D}{\partial w}\left(\frac{\partial w}{\partial r}\right)^{2}+\frac{\partial D}{\partial T} \frac{\partial w}{\partial r} \frac{\partial T}{\partial r}+\frac{\alpha}{\phi \mu} \frac{\partial w}{\partial z}-\frac{\alpha w}{\phi \mu^{2}}\left(\frac{\partial \mu}{\partial w} \frac{\partial w}{\partial z}+\frac{\partial \mu}{\partial T} \frac{\partial T}{\partial z}\right)
$$

and,

$$
-\frac{\partial z}{\partial t}+f_{3}=G_{3}(t, r, z)=0
$$

where

$$
f_{3}=-\frac{\alpha}{\phi \bar{\mu}}
$$

Thus maximization of $I$ is equivalent to the maximization of the following augmented functional:

$$
J=I+\int_{0}^{t_{\mathrm{f}}} \int_{0}^{R} \int_{0}^{Z_{0}}\left[\lambda_{1}(t, r, z) G_{1}+\lambda_{2}(t, r, z) G_{2}+\frac{\lambda_{3}(t, r) G_{3}}{Z_{0}}\right] \mathrm{d} z \mathrm{~d} r \mathrm{~d} t
$$


where $\lambda_{1}, \lambda_{2}$ and $\lambda_{3}$ are the undetermined co-state variables corresponding to three state variables temperature, mass fraction (nitrogen) and axial distance respectively. The bound is subjected to Equation 4.4. Upon substituting Equations 4.5, 4.6 and 4.7 into 4.8, we get

$$
\begin{aligned}
J= & \int_{0}^{t_{\mathrm{f}}} \int_{0}^{R} \int_{0}^{Z_{0}}\left\{\frac{\lambda_{3}}{Z_{0}}\left(-\frac{\partial z}{\partial t}-\frac{\alpha}{\phi \bar{\mu}}\right)+\frac{\beta r}{\bar{\mu}}\right\} \mathrm{d} z \mathrm{~d} r \mathrm{~d} t \\
& +\int_{0}^{t_{\mathrm{f}}} \int_{0}^{R} \int_{0}^{Z_{0}} \lambda_{1}\left\{-\frac{\partial T}{\partial t}+\frac{\gamma}{r} \frac{\partial T}{\partial r}+\gamma \frac{\partial^{2} T}{\partial r^{2}}+\gamma \frac{\partial^{2} T}{\partial z^{2}}+\frac{\alpha}{\phi \mu} \frac{\partial T}{\partial z}-\frac{\alpha T}{\phi \mu^{2}}\left(\frac{\partial \mu}{\partial w} \frac{\partial w}{\partial z}\right.\right. \\
& \left.\left.+\frac{\partial \mu}{\partial T} \frac{\partial T}{\partial z}\right)\right\} \mathrm{d} z \mathrm{~d} r \mathrm{~d} t+\int_{0}^{t_{\mathrm{f}}} \int_{0}^{R} \int_{0}^{Z_{0}} \lambda_{2}\left\{-\frac{\partial w}{\partial t}+\frac{D}{r} \frac{\partial w}{\partial r}+D \frac{\partial^{2} w}{\partial r^{2}}+\frac{\partial D}{\partial w}\left(\frac{\partial w}{\partial r}\right)^{2}\right. \\
& \left.+\frac{\partial D}{\partial T} \frac{\partial w}{\partial r} \frac{\partial T}{\partial r}+\frac{\alpha}{\phi \mu} \frac{\partial w}{\partial z}-\frac{\alpha w}{\phi \mu^{2}}\left(\frac{\partial \mu}{\partial w} \frac{\partial w}{\partial z}+\frac{\partial \mu}{\partial T} \frac{\partial T}{\partial z}\right)\right\} \mathrm{d} z \mathrm{~d} r \mathrm{~d} t .
\end{aligned}
$$

Next step is to obtain variation of $J$ and deduce the necessary conditions for its maximum.

\section{Control Function}

Control function for this problem is interfacial temperature as a function of time which is $T_{\text {int }}(t)$. Referring to Figure 4.2 , interfacial temperature prevails at:

1. the curved surface, that is, $T(t, R, z)$ for any height and time;

2. the bottom surface, that is, $T(t, r, 0)$ for any radial distance and time; and

3. the top surface, that is, $T\left(t, r, Z_{0}\right)$ for any radial distance and time.

Basically, $T(t, R, z), T(t, r, 0)$, and $T\left(t, r, Z_{0}\right)$ represent the same entity $T_{\text {int }}(t)$ at different physical locations. Eventually, each variation $\delta T(t, R, z), \delta T(t, r, 0)$, and $\delta T\left(t, r, Z_{0}\right)$ represents the same variational entity or $\delta T_{\text {int }}(t)$. To be admissible, $T_{\text {int }}(t)$, should be such that the state equations, and the associated initial and boundary conditions are satisfied. 


\subsubsection{Necessary Conditions of Optimality}

The necessary condition for the maximum of $J$ is that its variation $\delta J$ be zero, that is,

$$
\begin{aligned}
\delta J=\delta I & +\int_{0}^{t_{\mathrm{f}}} \int_{0}^{R} \int_{0}^{Z_{0}}\left(\delta \lambda_{1} G_{1}+\lambda_{1} \delta G_{1}\right) \mathrm{d} z \mathrm{~d} r \mathrm{~d} t+\int_{0}^{t_{\mathrm{f}}} \int_{0}^{R} \int_{0}^{Z_{0}}\left(\delta \lambda_{2} G_{2}+\lambda_{2} \delta G_{2}\right) \mathrm{d} z \mathrm{~d} r \mathrm{~d} t \\
& +\int_{0}^{t_{\mathrm{f}}} \int_{0}^{R} \int_{0}^{Z_{0}} \frac{\left(\delta \lambda_{3} G_{3}+\lambda_{3} \delta G_{3}\right)}{Z_{0}} \mathrm{~d} z \mathrm{~d} r \mathrm{~d} t=0 .
\end{aligned}
$$

We obtain by necessity following three co-state equations:

Co-state Equation 1:

$$
\begin{aligned}
\frac{\partial \lambda_{1}}{\partial t}= & -\lambda_{1} \frac{\partial f_{1}}{\partial T}-\lambda_{2} \frac{\partial f_{2}}{\partial T}+\frac{\partial}{\partial r}\left(\lambda_{1} \frac{\partial f_{1}}{\partial T_{r}}\right)+\frac{\partial}{\partial z}\left(\lambda_{1} \frac{\partial f_{1}}{\partial T_{z}}\right)+\frac{\partial}{\partial r}\left(\lambda_{2} \frac{\partial f_{2}}{\partial T_{r}}\right) \\
& +\frac{\partial}{\partial z}\left(\lambda_{2} \frac{\partial f_{2}}{\partial T_{z}}\right)-\frac{\partial^{2}}{\partial r^{2}}\left(\lambda_{1} \frac{\partial f_{1}}{\partial T_{r r}}\right)-\frac{\partial^{2}}{\partial z^{2}}\left(\lambda_{1} \frac{\partial f_{1}}{\partial T_{z z}}\right)-\frac{\partial^{2}}{\partial r^{2}}\left(\lambda_{2} \frac{\partial f_{2}}{\partial T_{r r}}\right) \\
& -\frac{\partial^{2}}{\partial z^{2}}\left(\lambda_{2} \frac{\partial f_{2}}{\partial T_{z z}}\right)+\left(2 \pi r \rho-\frac{\lambda_{3}}{\phi}\right) \frac{\alpha}{Z_{0} \bar{\mu}^{2}} \frac{\partial \bar{\mu}}{\partial \bar{T}} \frac{\partial \bar{T}}{\partial T}
\end{aligned}
$$

and $0 \leq r \leq R$ and $0 \leq z \leq Z_{0}$

Co-state Equation 2:

$$
\begin{aligned}
\frac{\partial \lambda_{2}}{\partial t}= & -\lambda_{1} \frac{\partial f_{1}}{\partial w}-\lambda_{2} \frac{\partial f_{2}}{\partial w}+\frac{\partial}{\partial r}\left(\lambda_{1} \frac{\partial f_{1}}{\partial w_{r}}\right)+\frac{\partial}{\partial z}\left(\lambda_{1} \frac{\partial f_{1}}{\partial w_{z}}\right)+\frac{\partial}{\partial r}\left(\lambda_{2} \frac{\partial f_{2}}{\partial w_{r}}\right) \\
& +\frac{\partial}{\partial z}\left(\lambda_{2} \frac{\partial f_{2}}{\partial w_{z}}\right)-\frac{\partial^{2}}{\partial r^{2}}\left(\lambda_{1} \frac{\partial f_{1}}{\partial w_{r r}}\right)-\frac{\partial^{2}}{\partial z^{2}}\left(\lambda_{1} \frac{\partial f_{1}}{\partial w_{z z}}\right)-\frac{\partial^{2}}{\partial r^{2}}\left(\lambda_{2} \frac{\partial f_{2}}{\partial w_{r r}}\right) \\
& -\frac{\partial^{2}}{\partial z^{2}}\left(\lambda_{2} \frac{\partial f_{2}}{\partial w_{z z}}\right)+\left(2 \pi r \rho-\frac{\lambda_{3}}{\phi}\right) \frac{\alpha}{Z_{0} \bar{\mu}^{2}} \frac{\partial \bar{\mu}}{\partial \bar{w}} \frac{\partial \bar{w}}{\partial w},
\end{aligned}
$$

and $0 \leq r \leq R$ and $0 \leq z \leq Z_{0}$

Co-state Equation 3:

$$
\begin{aligned}
& \frac{\partial \lambda_{3}}{\partial t}=0, \\
& \quad \text { and } 0 \leq r \leq R
\end{aligned}
$$


and, one stationarity condition:

$$
\begin{aligned}
\frac{\partial J}{\partial T_{\mathrm{int}}}= & \left\{-\frac{1}{R}\left[\frac{\partial}{\partial r}\left(\lambda_{1} \frac{\partial f_{1}}{\partial w_{r r}}\right)\right]_{R}\left(\frac{\partial w_{\mathrm{int}}}{\partial T_{\mathrm{int}}}\right)-\frac{1}{R}\left[\frac{\partial}{\partial r}\left(\lambda_{1} \frac{\partial f_{1}}{\partial T_{r r}}\right)\right]_{R}\right. \\
& -\frac{1}{Z_{0}}\left[\frac{\partial}{\partial z}\left(\lambda_{1} \frac{\partial f_{1}}{\partial w_{z z}}\right)\right]_{Z_{0}}\left(\frac{\partial w_{\mathrm{int}}}{\partial T_{\mathrm{int}}}\right)+\frac{1}{Z_{0}}\left[\frac{\partial}{\partial z}\left(\lambda_{1} \frac{\partial f_{1}}{\partial w_{z z}}\right)\right]_{0}\left(\frac{\partial w_{\mathrm{int}}}{\partial T_{\mathrm{int}}}\right) \\
& -\frac{1}{Z_{0}}\left[\frac{\partial}{\partial z}\left(\lambda_{1} \frac{\partial f_{1}}{\partial T_{z z}}\right)\right]_{Z_{0}}+\frac{1}{Z_{0}}\left[\frac{\partial}{\partial z}\left(\lambda_{1} \frac{\partial f_{1}}{\partial T_{z z}}\right)\right]_{0} \\
& -\frac{1}{R}\left[\frac{\partial}{\partial r}\left(\lambda_{2} \frac{\partial f_{2}}{\partial w_{r r}}\right)\right]_{R}\left(\frac{\partial w_{\mathrm{int}}}{\partial T_{\mathrm{int}}}\right)-\frac{1}{R}\left[\frac{\partial}{\partial r}\left(\lambda_{2} \frac{\partial f_{2}}{\partial T_{r r}}\right)\right]_{R} \\
& -\frac{1}{Z_{0}}\left[\frac{\partial}{\partial z}\left(\lambda_{2} \frac{\partial f_{2}}{\partial w_{z z}}\right)\right]_{Z_{0}}\left(\frac{\partial w_{\mathrm{int}}}{\partial T_{\mathrm{int}}}\right)+\frac{1}{Z_{0}}\left[\frac{\partial}{\partial z}\left(\lambda_{2} \frac{\partial f_{2}}{\partial w_{z z}}\right)\right]_{0}\left(\frac{\partial w_{\mathrm{int}}}{\partial T_{\mathrm{int}}}\right) \\
& -\frac{1}{Z_{0}}\left[\frac{\partial}{\partial z}\left(\lambda_{2} \frac{\partial f_{2}}{\partial T_{z z}}\right)\right]_{Z_{0}}+\frac{1}{Z_{0}}\left[\frac{\partial}{\partial z}\left(\lambda_{2} \frac{\partial f_{2}}{\partial T_{z z}}\right)\right]_{0}+\frac{\lambda_{3}}{Z_{0}} \frac{\partial f_{3}}{\partial \bar{w}}\left(\frac{\partial \bar{w}}{\partial w_{\mathrm{int}}}\right)\left(\frac{\partial w_{\mathrm{int}}}{\partial T_{\mathrm{int}}}\right) \\
& \left.+\frac{\lambda_{3}}{Z_{0}} \frac{\partial f_{3}}{\partial \bar{T}}\left(\frac{\partial \bar{T}}{\partial T_{\mathrm{int}}}\right)-\frac{\beta r}{Z_{0} \cdot \bar{\mu}^{2}}\left[\frac{\partial \bar{\mu}}{\partial \bar{w}}\left(\frac{\partial \bar{w}}{\partial w_{\mathrm{int}}}\right)\left(\frac{\partial w_{\mathrm{int}}}{\partial T_{\mathrm{int}}}\right)+\frac{\partial \bar{\mu}}{\partial \bar{T}}\left(\frac{\partial \bar{T}}{\partial T_{\mathrm{int}}}\right)\right]\right\}=0
\end{aligned}
$$

where $\frac{\partial J}{\partial T_{\mathrm{int}}}$ is the variational derivative of $J$ with respect to $T_{\mathrm{int}}$. This derivative essentially approaches zero upon minimization of the augmented functional $J$. Detailed derivation of these equations is explained in Appendix $\mathrm{G}$.

\section{Terminal and Boundary Conditions for Co-state variables}

At the final time, state variables become fixed. Thus, terminal conditions at $t=t_{\mathrm{f}}$ are:

$$
\begin{aligned}
& \lambda_{1,2}=\left\{\begin{array}{l}
0 \quad \forall 0<z<Z_{0}, 0<r<R \\
0 \quad \text { at } r=R \forall 0 \leq z \leq Z_{0} \text { and } z \in\left\{0, Z_{0}\right\} \forall 0 \leq r<R,
\end{array}\right. \\
& \lambda_{3}=0 \quad \forall 0 \leq r \leq R .
\end{aligned}
$$

The boundary conditions at any point of time $0<t<t_{\mathrm{f}}$ are

$$
\lambda_{1,2}=0 \quad \text { at } r=R \forall 0 \leq z \leq Z_{0} \text { and } z \in\left\{0, Z_{0}\right\} \forall 0 \leq r<R
$$


Additionally, due to symmetry,

$$
\frac{\partial \lambda_{1}}{\partial r}, \frac{\partial \lambda_{2}}{\partial r}=0 \quad \text { at } r=0 \forall 0 \leq z \leq Z_{0}
$$

Summing up, the necessary conditions for the maximum of $J$ are

1. the state equations (Equation 4.1. Equation 4.2 and Equation 4.3), and the set of initial and boundary conditions,

2. the co-state equations (Equation 4.11, Equation 4.12 and Equation 4.13), and the set of terminal and boundary conditions; and

3. the stationarity condition (Equation 4.14).

The aforementioned conditions must be satisfied in order for $T_{\mathrm{int}}(t)$ to be optimal, that is, maximize $I$ (or, $J$ ) or the oil production.

\subsubsection{Closed Loop Optimal Control Framework}

Following the development of Chapter 3, fully discretized Hamiltonian (at any instant of time $t$ ) in our case is given by

$$
\begin{aligned}
H=\sum_{i=0}^{L-1} & \left(2 \pi \rho r_{i}-\frac{\lambda_{3 i}}{\phi}\right) \frac{\alpha}{\bar{\mu}} \Delta r+\sum_{i=0}^{L-1} \sum_{j=0}^{M-1} \lambda_{1 i j}\left[\gamma\left(\frac{T_{r i j}}{r_{i}}+T_{r r i j}+T_{z z i j}\right)+\frac{\alpha T_{z i j}}{\phi \mu_{i j}}\right. \\
& \left.-\frac{\alpha T_{i j}}{\phi \mu_{i j}^{2}}\left(\mu_{w i j} w_{z i j}+\mu_{T i j} T_{z i j}\right)\right] \Delta r \Delta z+\sum_{i=0}^{L-1} \sum_{j=0}^{M-1} \lambda_{2 i j}\left[D_{i j}\left(\frac{w_{r i j}}{r_{i}}+w_{r r i j}\right)\right. \\
+ & \left.D_{w i j} w_{r i j}^{2}+D_{T i j} w_{r i j} T_{r i j}+\frac{\alpha w_{z i j}}{\phi \mu_{i j}}-\frac{\alpha w_{i j}}{\phi \mu_{i j}^{2}}\left(\mu_{w i j} w_{z i j}+\mu_{T i j} T_{z i j}\right)\right] \Delta r \Delta z .
\end{aligned}
$$

In this case, both $T_{i j}$ and $w_{i j}$ have dimensions of $N_{i} \times N_{j}$ whereas, $z_{i}$ entails $N_{i}$ numbers of grid points as it varies radially only. Moreover, $\mathbf{f}=\left[\begin{array}{lll}f_{1} & f_{2} & f_{3}\end{array}\right]^{\top}$. Also, following Table 4.1 displays the dimensions of relevant matrices for our algorithm: 
Table 4.1: Matrix Dimensions

\begin{tabular}{|c|c|c|c|c|c|c|}
\hline matrix & $\mathbf{S}$ & $\mathbf{A}$ & $\mathbf{B}$ & $\mathbf{C}$ & $\mathbf{D}$ & $\mathbf{E}$ \\
\hline dimension & $n \times n$ & $n \times n$ & $n \times 1$ & $n \times n$ & $1 \times 1$ & $n \times n$ \\
\hline
\end{tabular}

\section{Terminal and Boundary Conditions for Riccati variables}

For integrating system of Riccati equations (Equation 3.26) backward, we utilize optimal state and optimal co-state and deploy following terminal and boundary conditions. At the final time, state variables become fixed. Terminal conditions at $t=t_{\mathrm{f}}$ are:

$$
S_{i j}= \begin{cases}0 & \forall 0<z<Z_{0}, 0<r<R \\ 0 & \text { at } r=R \forall 0 \leq z \leq Z_{0} \text { and } z \in\left\{0, Z_{0}\right\} \forall 0 \leq r<R,\end{cases}
$$

The boundary conditions at any point of time $0<t<t_{\mathrm{f}}$ are

$$
S_{i j}=0 \text { at } r=R \forall 0 \leq z \leq Z_{0} \text { and } z \in\left\{0, Z_{0}\right\} \forall 0 \leq r<R \text {, }
$$

Moreover, due to symmetry,

$$
\frac{\partial S_{i j}}{\partial r}=0 \quad \text { at } r=0 \forall 0 \leq z \leq Z_{0} .
$$

\subsection{Development of Computer Program}

We develop the computer program using $\mathrm{C}++$ programming language. We solve (integrate) model equations, then we obtain open loop policies $\left[T_{\text {int }}(t)\right]$ at different pressures and finally, we simulate for closed loop algorithm whenever there is a pressure upset.

\section{Model Equations}

We utilize spline fittings to interpolate and extrapolate interfacial concentration $\left(w_{\text {int }}\right)$ and diffusivity $(D)$ at different grid point locations of temperatures and mass fraction respectively. For example, we have four different data sets for $w_{\text {int }}$ versus $T$ at five different pressures as 
described in Figure??? Hence, we use quadratic spline for interpolating solubility at different intermediate temperature at any pressure as number of data points is four. On the other hand, we have thirty data points for $D$ versus $w$ at a given temperature and pressure. In this case, we fit cubic spline for interpolation purposes. We have developed two separate libraries (for quadratic and cubic spline) libraries for these purposes.

We fully discretize partial differential equations along radial and axial directions; thus, they are converted in to ordinary differential equations and we integrate them using RungeKutta-Fehlberg method with adaptive step-size. We have developed and deployed two different libraries for these purposes. Adaptive step size is evaluated in one library whereas, Runge-Kutta-Fehlberg algorithm is coded in the other library. In addition, we deploy Simpson's rule for numerical integration purposes.

\section{Open Loop Policies and Riccati Equations}

For open loop optimal control, we generate a library for gradient descent line search method and for unconstrained minimization using Broyden-Fletcher-Goldfarb-Shanno algorithm. These two techniques were incorporated within the same library as two separate classes; moreover this library was included in the main model for optimizing the objective functional. We write state equations, co-state equations, stationarity condition and the objective functional in four separate functions and fully discretize them for different grid points. As per the computational algorithm described in section 2.7, we utilize the same library for maximizing the objective functional at different pressures. Eventually, we obtain open loop policies as an output of this library. As soon as we obtain a distinguished policy, we store optimal state, co-state and control using cubic splines with respect to time.

Both differential equation solver libraries (same were deployed for open loop simulation) are utilized for solving Riccati equations as well. We take derivatives of fully discretized co-state equations (section 4.2.3) and the Hamiltonian (section 4.2.4) with respect to $\mathbf{y}_{i j}$ and $\mathbf{u}_{i j}$ and obtain different matrices of Riccati equation at different grid point locations. Then, we populate these matrices and re-organize them in the form of Equation 3.26. In 
addition, we utilize the stored splines for optimal state and co-state variables to integrate these equations along with interpolated $D$ and $w_{\text {int }}$ at different pressures.

\section{Close Loop Simulation}

By solving model equations, open loop policies and Riccati equations at different pressures, we obtain our repository of optimal state, optimal gain and optimal control. We deploy this repository along with $w_{\text {int }}(T, P)$ and $D(w, T, P)$ data sets for the closed loop simulation. Particularly for closed loop simulation, we develop an adjustment library that encompasses different interpolation/adjust classes for diffusivity $(D)$, interfacial concentration $\left(w_{\text {int }}\right)$ and gain $(K)$ at different pressure points. Different data files comprising $w_{\text {int }}(T, P), \mathbf{K}(\mathbf{y}, P)$ and $D(w, T, P)$ and are rigorously checked and validated within these classes. All interpolations are performed using spline fitting libraries.

The main purpose is to adjust and interpolate these parameters whenever there is a pressure upset. Hence, at tweaked boundary condition (pressure), our model library utilizes adjusted $\mathbf{K}, D$ and $w_{\text {int }}$ to integrate state equations. Then the control function is adjusted to its optimal points at new pressure with the aid of the closest (interpolated) gain parameter using relevant functions in model library. All these aforementioned libraries and classes are deployed for open loop and closed loop simulations and results are portrayed in section 4.4 .

\subsection{Simulation Results}

At the onset, we evaluate diffusivity $D(w)$ of nitrogen as a function of solvent mass fraction at different temperature $(T)$ and pressure $(P)$. Both solubility data (Appendix D.1) and diffusivity data (Appendix C.2) are deployed in the simulation studies. In this section, we discuss following simulation results:

1. We generate open loop optimal control policies at five different pressures $(74.5 \mathrm{psi}$, $58.5 \mathrm{psi}, 50 \mathrm{psi}, 43.5 \mathrm{psi}$ and $24.5 \mathrm{psi})$ and create our repository of optimal variables optimal state, optimal control and optimal gain. 
2. We discuss the effectiveness of the state-feedback proportional gain control law in presence of pressure upset and explain different observations.

We performed all the simulations and observed different phenomena for a span of $163.85 \mathrm{~min}$.

\subsubsection{Open Loop Simulation Results}

This section congregates the results of open loop optimal control strategy and open loop simulation results:

1. We explain the open loop optimal control strategy.

2. We discuss the effects of different initial guesses on the optimal policies at different pressures.

3. We analyze the effects of selecting different grid points on the final open loop policies.

4. We describe iterative convergence of the objective functional.

5. We conclude the section with the analysis of the open loop optimal control policies at different pressures.

\section{Computational Algorithm to Determine Optimal $T_{\text {int }}(t)$}

The necessary conditions contain non-linear partial differential equations encompassing split boundaries whose analytical solution is not possible. Therefore, this optimal control problem needs to be solved numerically. The computational algorithm, which was utilized to determine the optimal $T_{\text {int }}$ is as follows:

1. We provide an initial guess for $T_{\mathrm{int}}$ at each grid point in the stipulated time interval.

2. We integrate the state equations forward using the initial and boundary conditions and $T_{\text {int }}$ values. Then, we save different values of state variables $(T, w$ and $z)$ at each grid point in the space of $t, r$, and $z$. 
3. Thereafter, we evaluate the objective functional; in case, it does not increase after the initial iteration, we quit.

Table 4.2: Simulation Parameters

\begin{tabular}{|l|c|c|}
\hline Parameters & Value & Reference \\
\hline \hline number of grid points along t & 30 & Simulation \\
\hline number of grid points along r, $N_{i}$ & 6 & Simulation \\
\hline number of grid points along z, $N_{j}$ & 6 & Simulation \\
\hline diameter of the physical model, $d$ & $5.5 \mathrm{~cm}$ & Experiment \\
\hline initial height of the physical model, $Z_{0}$ & $35 \mathrm{~cm}$ & Experiment \\
\hline permeability, $K$ & $4 \mathrm{Darcy}$ & Experiment \\
\hline relative permeability, $K_{\mathrm{r}}$ & 1 & Reference [54] \\
\hline oil density, $\rho$ & $0.821 \mathrm{~g} \mathrm{~cm}^{-3}$ & Reference [54] \\
\hline gravity, $g$ & $35,31,600 \mathrm{~cm} \mathrm{~min}^{-2}$ & Reference [54] \\
\hline specific heat, $C_{\mathrm{p}}$ & $2.13 \mathrm{~J} \mathrm{~g}^{-1}{ }^{\circ} \mathrm{C}^{-1}$ & Reference [54] \\
\hline thermal conductivity, $k$ & $0.6 \mathrm{~J} \mathrm{~cm}^{-1} \mathrm{~min}^{-1}{ }^{\circ} \mathrm{C}^{-1}$ & Reference [54] \\
\hline
\end{tabular}

4. Upon increment in the value of the objective functional, we integrate co-state equations backward using terminal and boundary conditions, and the values of $T_{\mathrm{int}}$ and state variables. Thus, we save the values of co-state variables $\left(\lambda_{1}, \lambda_{2}\right.$ and $\left.\lambda_{3}\right)$ at each grid point in the space of $t, r$, and $z$.

5. Thereafter, we obtain new and improved values of $T_{\text {int }}$ at each grid point in the time interval using the variational derivative as follows: 
$T_{\text {int }}^{\text {new }}=T_{\text {int }}+\delta T_{\text {int }}$, where $\delta T_{\text {int }}=\epsilon \times\left(\frac{\partial J}{\partial T_{\text {int }}}\right)$ and $\epsilon$ is a small positive number whose optimal value provides maximum reduction in variation in the objective functional (as described in Section 2.77.

6. Using $T_{\text {int }}^{\text {new }}$, we repeat the computation Step 2 onward until there is no further significant improvement in the objective functional or when the improvement is negligible, the $T_{\text {int }}^{\text {new }}$ values are optimal.

We applied second order finite-difference approximations to the partial differential equations along the radial and axial directions in order to obtain a set of ordinary differential equations for each PDE at the grid points.

Table 4.3: Parameters used in Computations

\begin{tabular}{|l|c|}
\hline Parameters & Value \\
\hline \hline maximum number of iterations & 300 \\
\hline initial step-size for integration & $10^{-5}$ \\
\hline minimum step-size for integration & $10^{-8}$ \\
\hline maximum step-size for integration & 1 \\
\hline maximum step-length for line search & 0.1 \\
\hline tolerance on integration accuracy & $10^{-5}$ \\
\hline tolerance on gradient correction & $10^{-6}$ \\
\hline
\end{tabular}

These ODEs are very stiff to integrate. We used implicit Runge-Kutta-Fehlberg method with adaptive step-size to integrate the same. The Broyden-Fletcher-Goldfarb-Shanno method [50] was utilized to provide the improvements $\delta T_{\text {int }}$ based on the variational derivative. Table 4.2 incorporates the computational geometry and different parameters used for 
this simulation (For SI units, refer to Nomenclature). The computational algorithm was programmed in $\mathrm{C}++$ (using Visual Studio IDE) and executed on Intel Core-I7 processor (64 bit, $3.60 \mathrm{GHz}, 15.9 \mathrm{~GB}$ usable RAM). The parameter values are provided in Table 4.3 and were used in the determination of optimal $\delta T_{\text {int }}$ values at each minute of the time interval. These optimal policy along with optimal state and co-state variables are to be used as benchmarks for investigating different closed loop results. In addition, optimal state and co-state variables at five different pressures are stored in a repository which is to be utilized for interpolating the optimal profiles whenever there is an upset in the boundary conditions (pressure in our case).

\section{Effect of different initial guesses}

In the computation, we utilized different initial guesses (initial control function or, $T_{\text {int }}$ versus t) as portrayed in Figure 4.3 and Figure 4.4 .

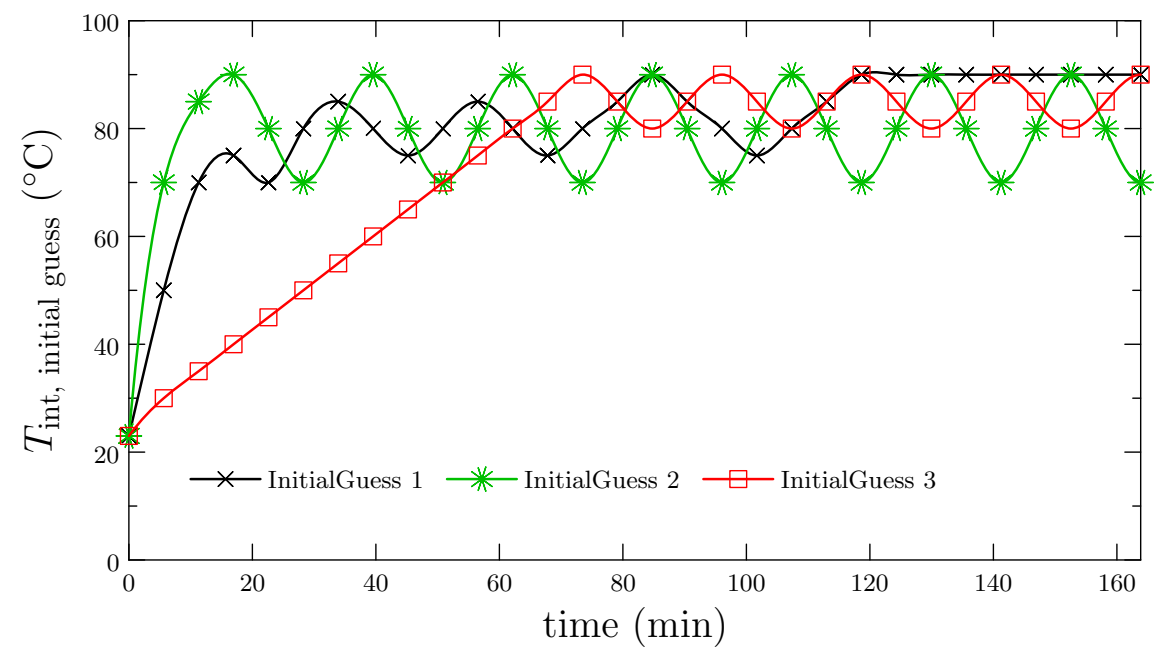

Figure 4.3: Initial guess for control function - set 1.

In Figure 4.3, we take wavy functions only whereas, in Figure 4.4, we consider linear functions as well. In some cases (Initial guess 3), we have considered a combination of both linear and wavy functions. The wavy functions differ in terms of amplitude and linear functions vary in terms of slope only. 


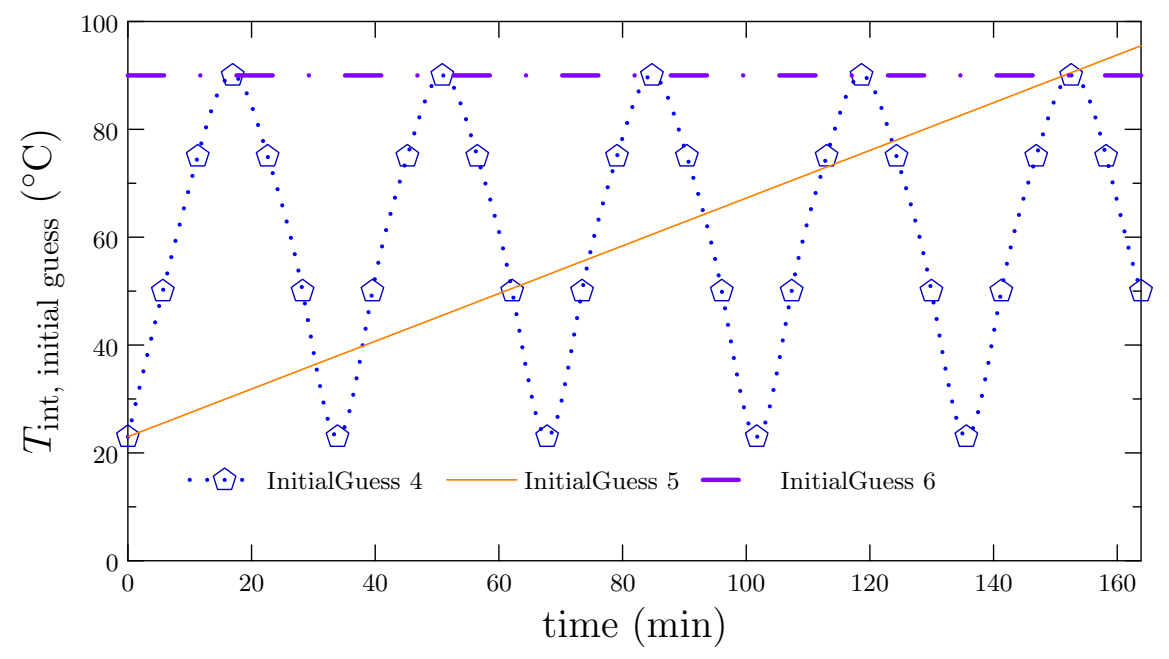

Figure 4.4: Initial guess for control function - set 2 .

We feed these initial guesses and simulate for the open loop converged optimal control policies. We do not observe any noteworthy trend in the number of iterations for obtaining the convergence. In other words, initial guesses do not effect the speed of convergence (computation time) at all. Overall, we attain computational convergence within 40 iterations.

For all the initial guesses, final converged optimal control policies nearly coincide for a given operating pressure. In any case, the converged policies are mild wavy in nature. Moreover, at 74.5 psi and 43.5 psi pressures, we hardly observe any difference in the policies except for the wavy patterns (as shown in Figure 4.5 and Figure 4.6). Even though we see slight differences in the converged policies for few of the cases (corresponding to 24.5 psi, 50 psi and $58.5 \mathrm{psi}$ ), the average temperature (of the final converged policies) and final objective functional values (mass of heavy oil recovered) are approximately same. Table 4.4 and Table 4.5 corroborates the aforementioned fact.

In a nutshell, we conclude that our open loop optimal control problem is independent of the choice of the initial guess for the control function. However, in this report we have showcased our closed-loop simulation results with respect to initial guess 2 which is wavy throughout the selected observation period of $163.85 \mathrm{~min}$ and the average temperature (of the guessed policy) is nearly $80^{\circ} \mathrm{C}$. 


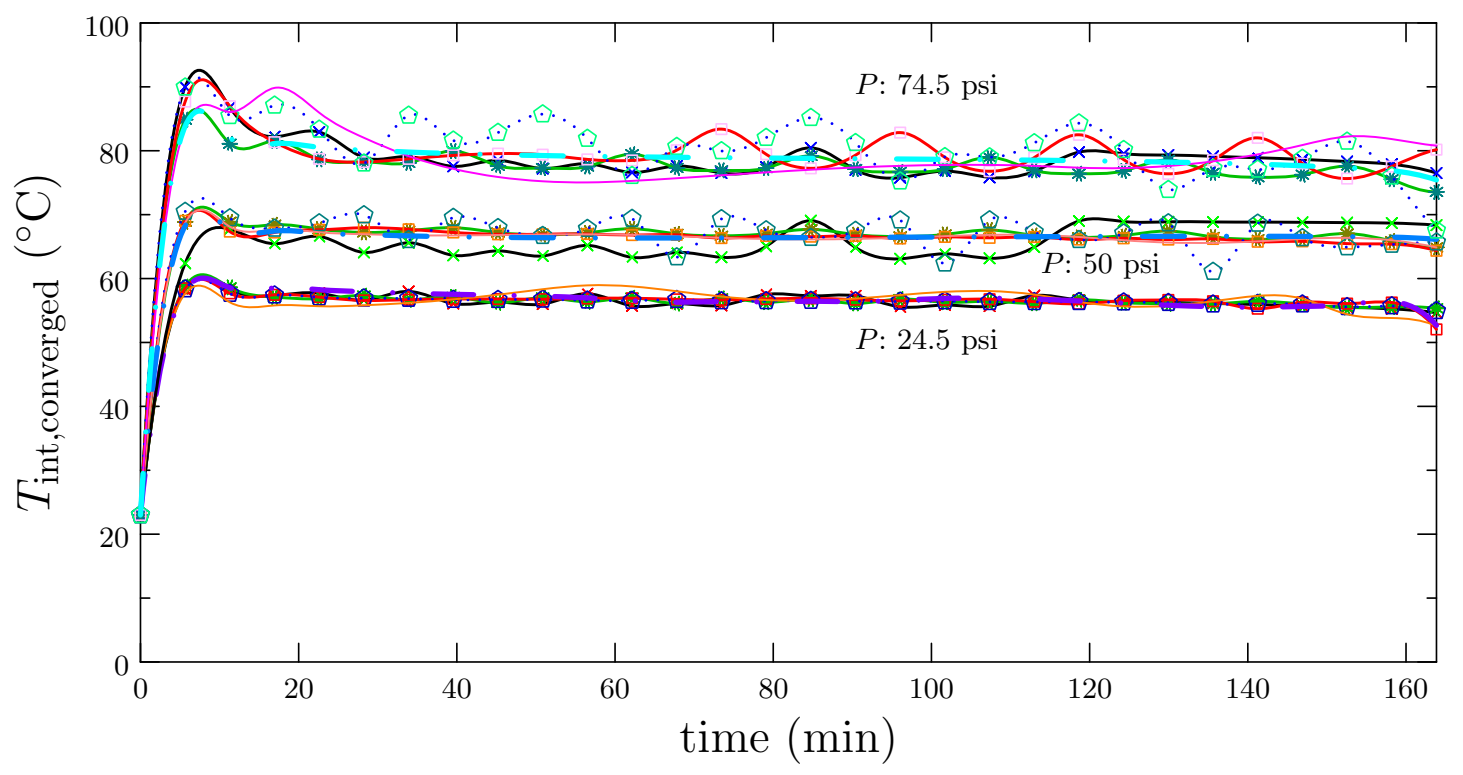

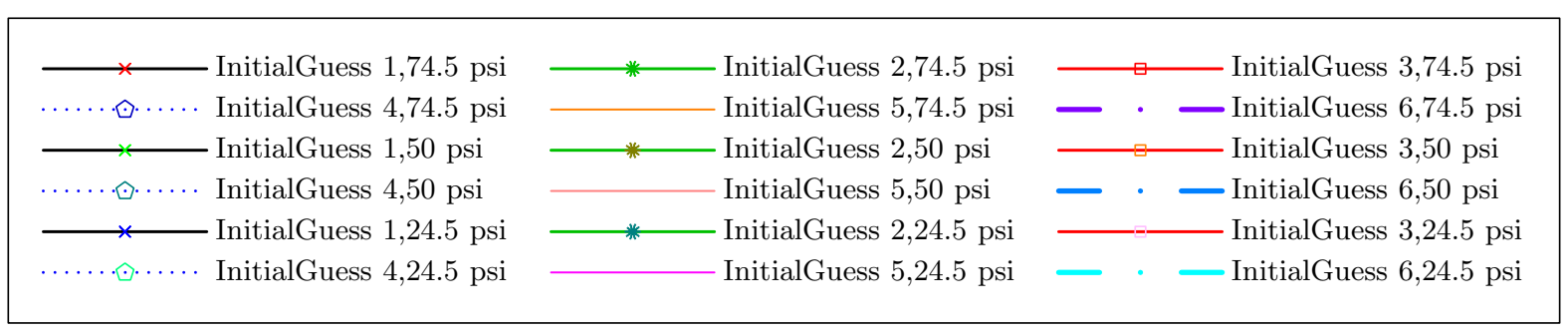

Figure 4.5: Results for $24.5 \mathrm{psi}, 50 \mathrm{psi}$ and $74.5 \mathrm{psi}$.

Table 4.4: Objective functionals for different initial guesses - set 1

\begin{tabular}{|c|c|c|c|}
\hline pressure (psi) & Initial guess 1 & Initial guess 2 & Initial guess 3 \\
\hline \hline 24.5 & 19.28 & 19.28 & 19.28 \\
\hline 43.5 & 34.99 & 34.99 & 34.99 \\
\hline 50.0 & 42.61 & 42.63 & 42.63 \\
\hline 58.5 & 52.75 & 52.75 & 52.75 \\
\hline 74.5 & 56.52 & 56.57 & 56.55 \\
\hline
\end{tabular}




\section{CHAPTER 4. APPLICATION}
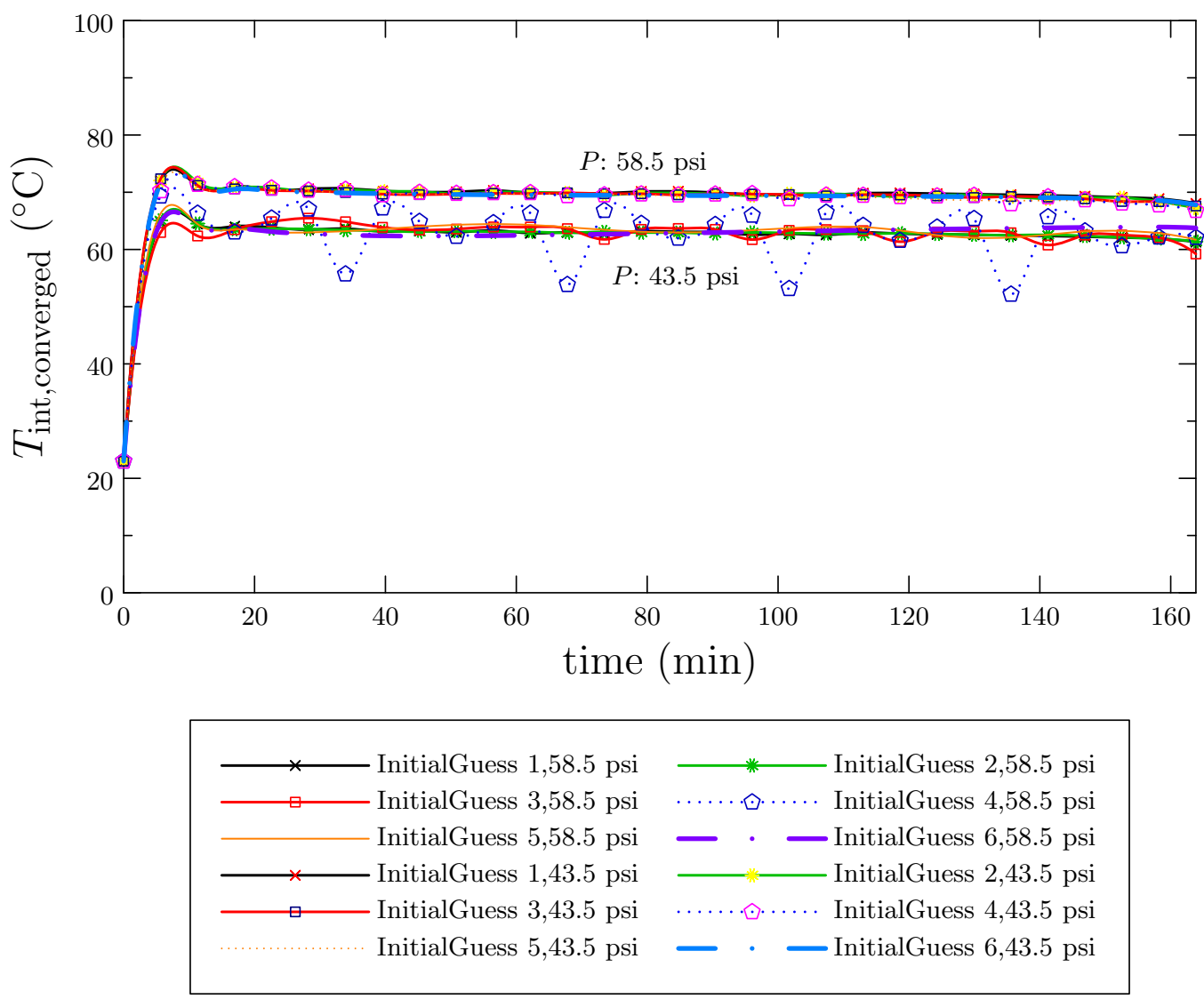

Figure 4.6: Results for 43.5 psi and 58.5 psi.

Table 4.5: Objective functionals for different initial guesses - set 2

\begin{tabular}{|c|c|c|c|}
\hline pressure (psi) & Initial guess 4 & Initial guess 5 & Initial guess 6 \\
\hline \hline 24.5 & 19.28 & 19.28 & 19.28 \\
\hline 43.5 & 34.98 & 34.98 & 34.99 \\
\hline 50.0 & 42.62 & 42.63 & 42.65 \\
\hline 58.5 & 52.70 & 52.75 & 52.77 \\
\hline 74.5 & 56.51 & 56.65 & 56.69 \\
\hline
\end{tabular}




\section{Effect of different grid points}

We have utilized different grid points to investigate open loop optimal policies with the corresponding objective functional.

Table 4.6: Grid point analysis

\begin{tabular}{|c|c|c|c|c|c|}
\hline pressure (psi) & grid point & iterations & objective functional $(\mathrm{g})$ & \%variation & step size \\
\hline \multirow{3}{*}{24.5} & $i=5, j=5$ & 18 & 20.07 & - & $10^{-3}$ \\
\hline & $i=6, j=6$ & 21 & 19.28 & 3.95 & $10^{-4}$ \\
\hline & $i=7, j=7$ & \multicolumn{4}{|c|}{ Equations become stiff; step size order $10^{-9}$} \\
\hline \multirow{3}{*}{43.5} & $i=5, j=5$ & 11 & 36.41 & - & $10^{-3}$ \\
\hline & $i=6, j=6$ & 16 & 34.99 & 3.91 & $10^{-4}$ \\
\hline & $i=7, j=7$ & \multicolumn{4}{|c|}{ Equations become stiff; step size order $10^{-9}$} \\
\hline \multirow{3}{*}{50.0} & $i=5, j=5$ & 11 & 44.36 & - & $10^{-3}$ \\
\hline & $i=6, j=6$ & 17 & 42.63 & 3.90 & $10^{-4}$ \\
\hline & $i=7, j=7$ & \multicolumn{4}{|c|}{ Equations become stiff; step size order $10^{-9}$} \\
\hline \multirow{3}{*}{58.5} & $i=5, j=5$ & 17 & 54.88 & - & $10^{-3}$ \\
\hline & $i=6, j=6$ & 27 & 52.75 & 3.87 & $10^{-4}$ \\
\hline & $i=7, j=7$ & \multicolumn{4}{|c|}{ Equations become stiff; step size order $10^{-9}$} \\
\hline \multirow{3}{*}{74.5} & $i=5, j=5$ & 13 & 60.23 & - & $10^{-3}$ \\
\hline & $i=6, j=6$ & 20 & 58.29 & 3.86 & $10^{-4}$ \\
\hline & $i=7, j=7$ & \multicolumn{4}{|c|}{ Equations become stiff; step size order $10^{-9}$} \\
\hline
\end{tabular}

Table 4.6 shows that there is no significant variation in the objective functional value from 
$(i=5, j=5)$ grid points to $(i=6, j=6)$ grid points (integration step size increased from $10^{-3}$ to $\left.10^{-4}\right)$. But $(i=7, j=7)$ combination makes the open loop solution computationally intensive as integration step size becomes of the order of $10^{-9}$. Eventually, CPU time becomes high and we prefer to stick with $(i=6, j=6)$ grid points for further analysis.

\section{Open loop solution convergence}

It takes up to 27 iterations (as shown in Table 4.7) for the algorithm to converge.

Table 4.7: Open Loop Results

\begin{tabular}{|c|c|c|c|}
\hline pressure (psi) & iterations & oil mass (g) & CPU time (min) \\
\hline \hline 24.5 & 21 & 19.28 & 20.3 \\
\hline 43.5 & 16 & 34.99 & 18.1 \\
\hline 50.0 & 17 & 42.63 & 18.8 \\
\hline 58.5 & 27 & 52.75 & 23.0 \\
\hline 74.5 & 20 & 58.29 & 21.4 \\
\hline
\end{tabular}

For the physical model with aforementioned geometry, upon convergence, we obtain optimal $T_{\text {int }}(t)$ at each minute of the time interval. The optimal $T_{\text {int }}(t)$ values thus obtained are converted into the open loop optimal policy of nitrogen temperature versus time using the experimental data, $w_{\text {int }}=w_{\text {int }}\left(T_{\text {int }}\right)$ and diffusivity data $D(w, T)$ at 5 given pressure $(P)$ values ranging between 24.5 psi to 74.5psi (described in Appendix $\mathrm{D}$ and $\mathrm{C}$ respectively). Computation is initialized with the guessed $T_{\text {int }}$ versus time relation (second initial guess) at different pressure magnitudes. As the iterations proceed, the oil production increases monotonically. The improvement is significant during the initial iterations but slows down towards the end for any pressure. The convergence for 74.5 psi is portrayed in Figure 4.7. In all cases the computation converged within $25 \mathrm{~min}$. 


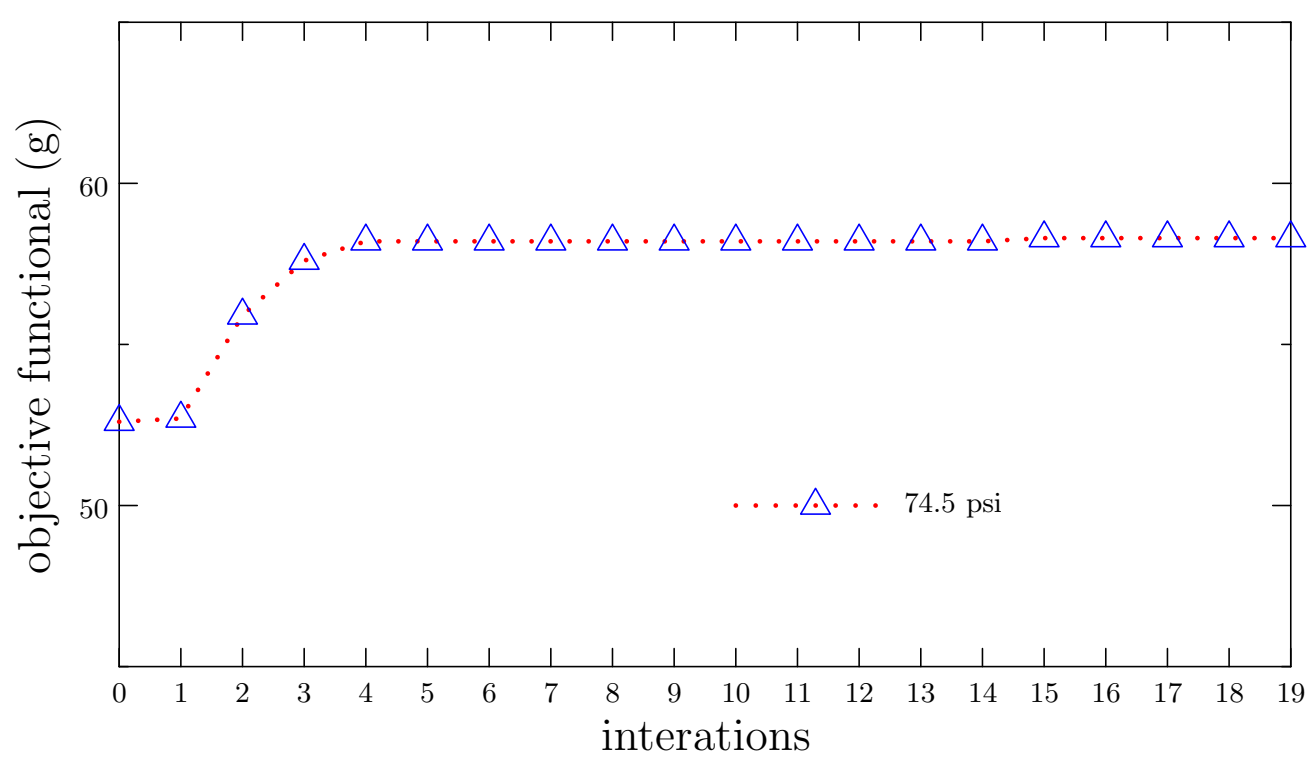

Figure 4.7: Convergence of objective functional.

\section{Open loop optimal policies}

The computation is performed for the physical model of 4 Darcy permeability and $35 \mathrm{~cm}$ drainage height and $5.5 \mathrm{~cm}$ of diameter and we observe different optimal policies for a span of $163.85 \mathrm{~min}$ (approximately). The algorithm converged in five different numbers of iterations at five different pressures yielding five different optimal $T_{\text {int }}(t)$ (policies) and five different objective functional values (mass of oil produced) as shown in Table 4.7 and Figure 4.8. Some crucial observations are:

1. As pressure increases from $24.5 \mathrm{psi}$ to $74.5 \mathrm{psi}$, value of the objective functional increases from $19.28 \mathrm{~g}$ to $58.29 \mathrm{~g}$ whereas, the average optimal $T_{\text {int }}(t)$ decreases from $77.90^{\circ} \mathrm{C}$ to $56.57^{\circ} \mathrm{C}$ as shown in Figure 4.8. Eventually, we presume that at higher pressure conditions, lower temperature is mandated to enhance heavy oil recovery.

2. The optimal $T_{\text {int }}(t)$ policies (converged final policies) are mild wavy in nature at any given pressure ranging between $24.5 \mathrm{psi}$ to $74.5 \mathrm{psi}$ in our observation period of $163.85 \mathrm{~min}$. 
3. Optimal policies at different pressures do not coincide or overlap to each other; distinct optimal $T_{\text {int }}(t)$ maximizes heavy oil recovery at different pressures.

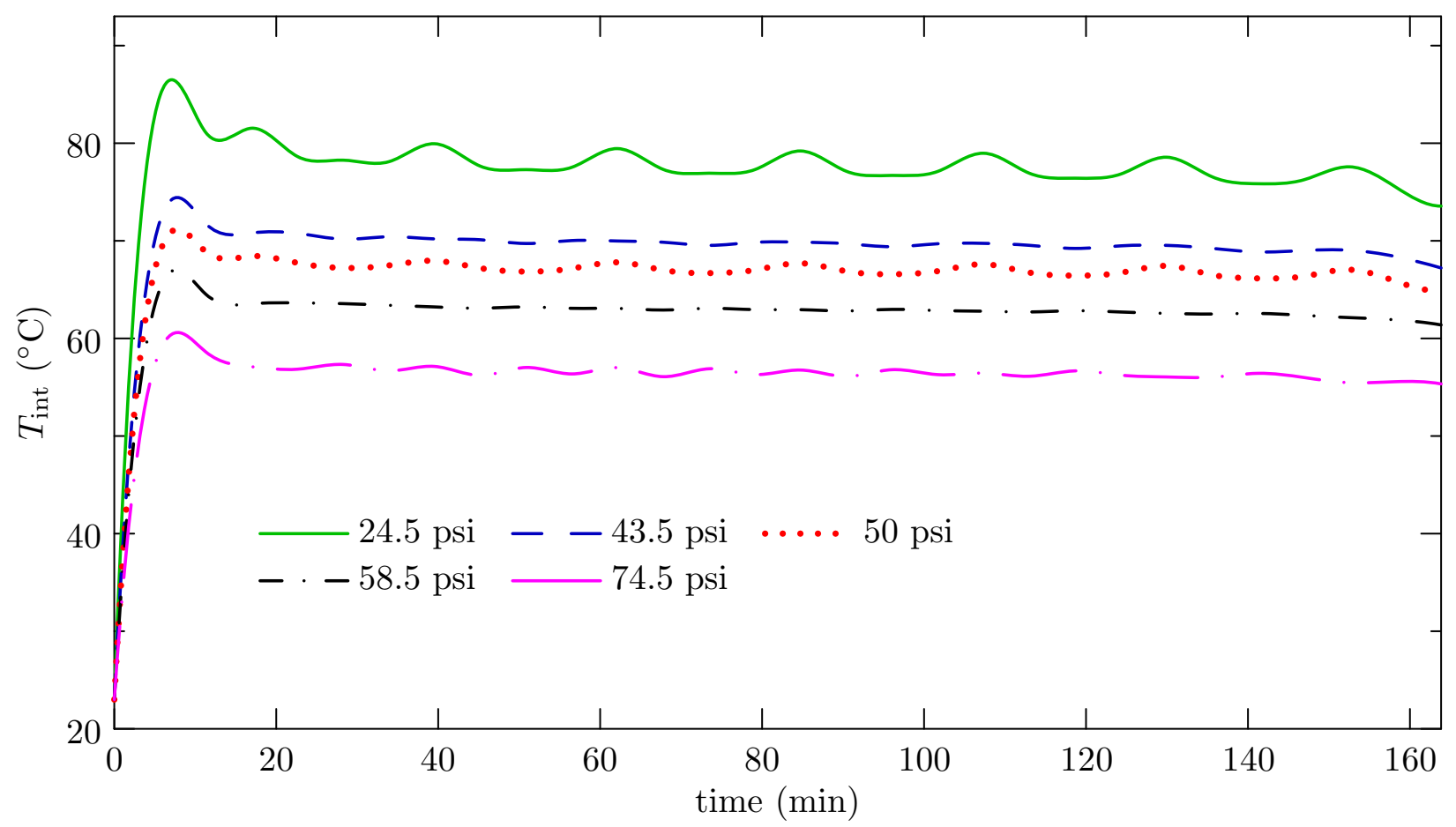

Figure 4.8: Optimal temperature policies at different pressures.

4. We can compare these policies with the experimental results as shown in Appendix D. It is observed that at $90^{\circ} \mathrm{C}$ (highest operating temperature) we recover $21.3 \mathrm{~g}, 36.2 \mathrm{~g}$, $43.7 \mathrm{~g}, 53.7 \mathrm{~g}$ and $66.5 \mathrm{~g}$ at $24.5 \mathrm{psi}, 43.5 \mathrm{psi}, 50 \mathrm{psi}, 58.5 \mathrm{psi}$ and $74.5 \mathrm{psi}$ respectively. But with the optimal policies, we are able to recover (theoretically) $19.3 \mathrm{~g}, 34.5 \mathrm{~g}$, $42.6 \mathrm{~g}, 52.8 \mathrm{~g}$ and $58.3 \mathrm{~g}$ at $24.5 \mathrm{psi}, 43.5 \mathrm{psi}, 50 \mathrm{psi}, 58.5 \mathrm{psi}$ and $74.5 \mathrm{psi}$ at an average temperature of $77.9^{\circ} \mathrm{C}, 69.8^{\circ} \mathrm{C}, 67.1^{\circ} \mathrm{C}, 62.9^{\circ} \mathrm{C}$ and $56.6^{\circ} \mathrm{C}$ respectively. In other words, we obtain more amount of heavy oil at lesser (optimal) temperature control.

From the validated process model (Appendix $\mathrm{E}$ and $\mathrm{F}$, we set these optimal $T_{\text {int }}(t)$ at five different pressures as our benchmarks and further we simulate and analyze closed loop results using the aforementioned optimal policies within the range of operating pressures. 


\subsubsection{Closed Loop Simulation Results}

In this section, first we describe close loop control methodology. Then we analyze the closed loop results by introducing step change in pressure at any intermediate operating time in the span of $163.85 \mathrm{~min}$.

\section{Closed Loop Optimal Control Methodology}

We utilize optimal state and optimal co-state for integrating non-linear distributed parameter system of Riccati equations (Equation 3.26) backward with the aforementioned (in Section 4.2.4 terminal and boundary conditions. Then, we determined gain parameter matrix at a given pressure. We repeated the same for five different pressures and we generated our desired repository in terms of optimal state, optimal gain and optimal control $\left[T_{\text {int }}(t)\right]$ for five given pressures ranging between $24.5 \mathrm{psi}$ and $74.5 \mathrm{psi}$ corresponding to a coarse grid of fixed, unperturbed initial conditions $\overline{\mathbf{y}}_{0}$ as described in Table 4.8 .

Table 4.8: Open Loop Repository

\begin{tabular}{|llllll|}
\hline Pressure (psi): & 24.5 & 43.5 & 50.0 & 58.5 & 74.5 \\
\hline \hline optimal state: & $\hat{\mathbf{y}}_{24.5}$ & $\hat{\mathbf{y}}_{43.5}$ & $\hat{\mathbf{y}}_{50.0}$ & $\hat{\mathbf{y}}_{58.5}$ & $\hat{\mathbf{y}}_{74.5}$ \\
\hline Riccati variables: & $\mathbf{S}_{24.5}$ & $\mathbf{S}_{43.5}$ & $\mathbf{S}_{50.0}$ & $\mathbf{S}_{58.5}$ & $\mathbf{S}_{74.5}$ \\
\hline optimal gain: & $\hat{\mathbf{K}}_{24.5}$ & $\hat{\mathbf{K}}_{43.5}$ & $\hat{\mathbf{K}}_{50.0}$ & $\hat{\mathbf{K}}_{58.5}$ & $\hat{\mathbf{K}}_{74.5}$ \\
\hline
\end{tabular}

We deploy this repository for interpolating closest optimal gain $\left[\widehat{\mathbf{K}}_{P}(t)\right]$ and optimal state $\left[\hat{\mathbf{y}}_{P}(t)\right]$ at tweaked pressure in the stored grid. On the other hand, we have diffusivity versus concentration (mass fraction) data and viscosity correlation at different temperatures and pressures. We interpolate both of them for integrating state equations so that we have the desired information in terms of system state $\left[\mathbf{y}_{P}(t)\right]$ which is not optimal at modified pressure $(P)$. Moreover, we calculate operating control [non-optimal, $\mathbf{u}_{P}(t)$ ] at this new 
pressure. Subsequently, proportional gain state-feedback control law takes control action that is given by

$$
\hat{\mathbf{u}}_{P}(t)=\mathbf{u}_{P}(t)+\widehat{\mathbf{K}}_{P}(t)\left[\mathbf{y}_{P}(t)-\hat{\mathbf{y}}_{P}(t)\right]
$$

and aids in bridging the gap between the desired optimal control policy $\left[\hat{\mathbf{u}}_{P}(t)\right]$ and the prevailing non-optimal $T_{\text {int }}\left[\mathbf{u}_{P}(t)\right]$ at the existing operating pressure.

In other words, control adjustment occurs and the same steer the process towards optimality at the prevailing boundary conditions [48, 50]. Moreover, we solve the Riccati equations and generate the repository of optimal variables (state, gain and control - total size of files $430 \mathrm{kB}$ ) to further deploy in closed loop simulation. Algorithm is described in Figure 3.1

\section{Closed Loop Simulation Results}

Initially, we investigate within two known pressure bounds as open loop results [both objective functional values and optimal $\left.T_{\mathrm{int}}(t)\right]$ are readily available and it becomes convenient enough to test the closed loop strategy. In the real time mode, we start the simulation at a given intermediate pressure of $50 \mathrm{psi}$ and after $23 \mathrm{~min}$, we introduce an upset of $17 \%$ (step change) and the new operating pressure becomes 58.5 psi. Figure 4.9 shows the benchmark optimal $T_{\text {int }}(t)$ at both $50 \mathrm{psi}$ and $58.5 \mathrm{psi}$; moreover, we observe that real time $T_{\text {int }}(t)$ initially concurs with the optimal $T_{\mathrm{int}}(t)$ at $50 \mathrm{psi}$, then it starts varying after $23 \mathrm{~min}$ and eventually (almost) coincides with optimal $T_{\text {int }}(t)$ at 58.5 psi.

Additionally, objective functional value gets raised from an expected $42.6 \mathrm{~g}$ (corresponding to $50 \mathrm{psi}$ when there are no upsets) to a value of $51.0 \mathrm{~g}$ which is close to $52.8 \mathrm{~g}$ (corresponds to 58.5 psi policy) for an observation time of $163.85 \mathrm{~min}$ (approximately). Therefore, we conclude that the closed loop optimal control strategy (upon interpolating with respect to the repository of optimal variables and adjustment to control function) is precisely tracking the desired optimal $T_{\text {int }}(t)$ along with objective functional at the tweaked pressure magnitude. The corrective measure is significant even in terms of the extent of time that is applied for 
the control adjustment.

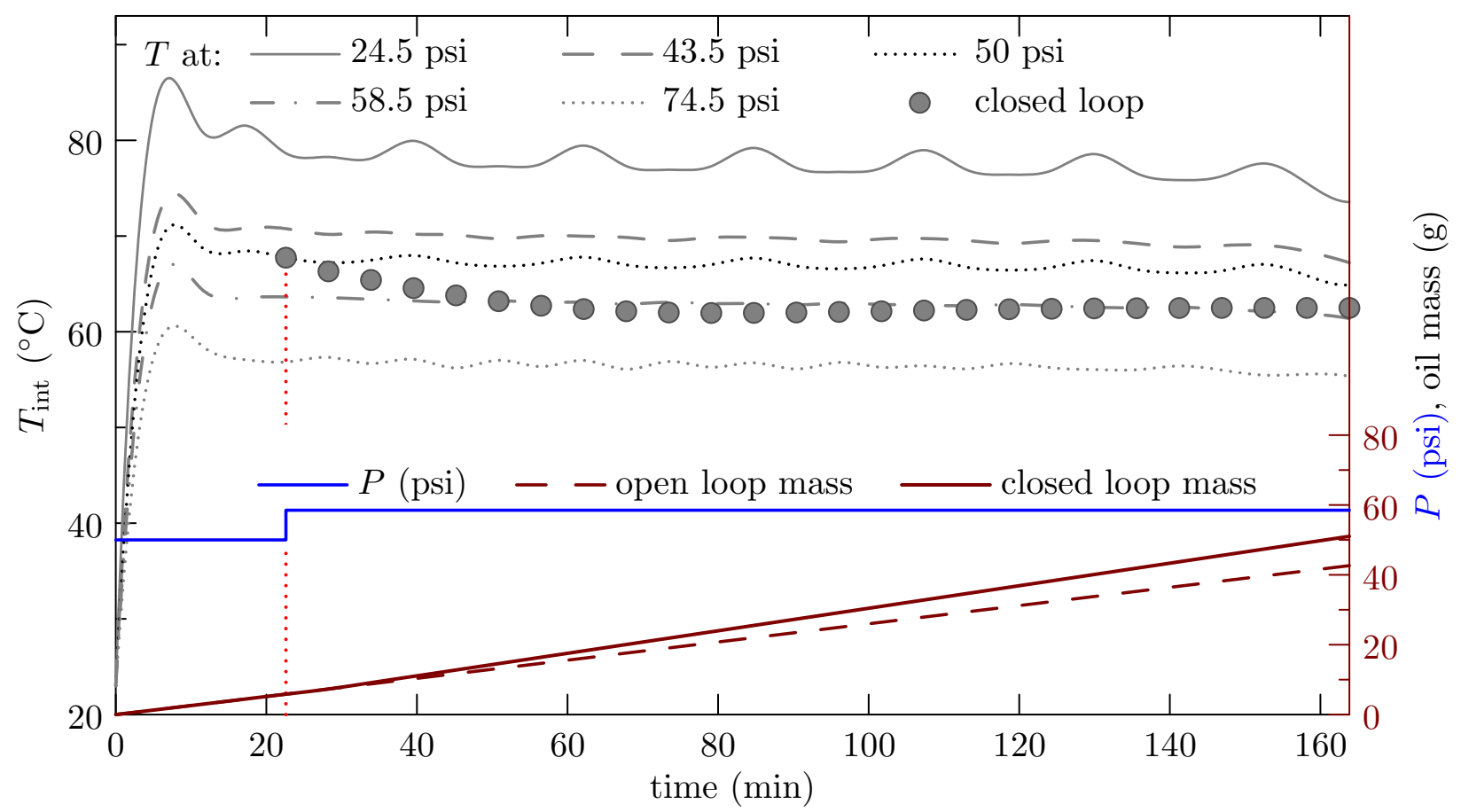

Figure 4.9: Closed Loop Result: Single upset within known bounds after 23 min.

Figure 4.10 depicts that for the similar investigation within the known bounds (as earlier) yields lesser mass (objective functional value) if the upset is induced after $73 \mathrm{~min}$. Though the final optimal $T_{\text {int }}(t)$ nearly coincides with the benchmark policy at 58.5 psi, the recovered mass is $48.0 \mathrm{~g}$. Thus, we deduce that our algorithm mandates sufficient (and necessary) adjustment time to orient towards new nominal solutions (based on interpolation) and steer the process towards desired optimality at new induced boundary condition.

Further, we investigate (and, validate) multiple upsets in terms of pressure as well. Starting with the same intermediate pressure of 50 psi, we induce upsets after $23 \mathrm{~min}, 28 \mathrm{~min}$, $39 \mathrm{~min}, 50 \mathrm{~min}$ and $56 \mathrm{~min}$ to new pressure values of $52.5 \mathrm{psi}, 56 \mathrm{psi}, 58.5 \mathrm{psi}, 62.5 \mathrm{psi}$ and 67.5 psi respectively as shown in Figure 4.11. The closed loop $T_{\text {int }}(t)$ policy initially tracks the policy corresponding to $50 \mathrm{psi}$ and then it start varying after $23 \mathrm{~min}$, right away the disturbance is introduced. Finally, $T_{\text {int }}(t)$ at 67.5 psi adjusts itself to an intermediate zone 
between 58.5 psi optimal policy and 74.5 psi optimal policy.

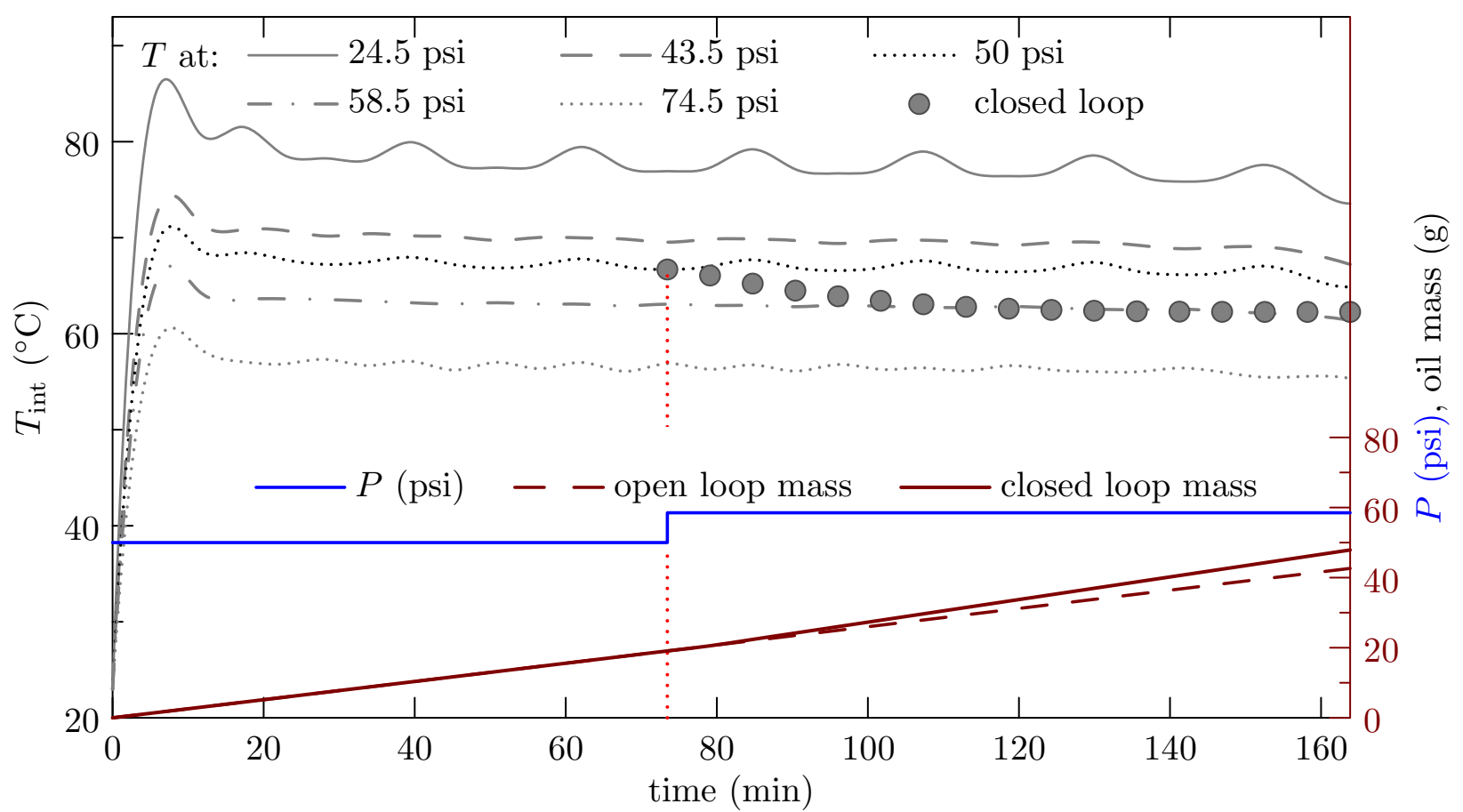

Figure 4.10: Closed Loop Result: Single upset within known bounds after 73 min.

Earlier, we observed that optimal $T_{\text {int }}(t)$ requirement becomes less as pressure rises; eventually, the result suffices our deduction as 67.5 psi optimal policy adjusts itself in between the bounds of 58.5 psi and 74.5 psi optimal policies. In addition, this simulation result satiates in terms of the objective functional value $(54.8 \mathrm{~g})$ also as it lies between objective functional values corresponding to $58.5 \mathrm{psi}(52.8 \mathrm{~g})$ and $74.5 \mathrm{psi}$ (58.3 g). Consequently, we conclude that the proportional gain control adjustment action manoeuvres the process towards optimality at new boundary condition in terms of operating pressure and stimulates enhanced oil recovery. We further extend our studies in terms of tracking the state property (temperature) at different grid points upon control adjustment. For the case with $17 \%$ pressure increment from the base value of 50 psi operating pressure, after 73 min we observe that, with time, whenever necessary control adjustment takes place, the state property gets manoeuvred towards the expected optimal state property corresponding to 58.5 psi psi at 
$(i=2$ and $j=3)$ grid points.

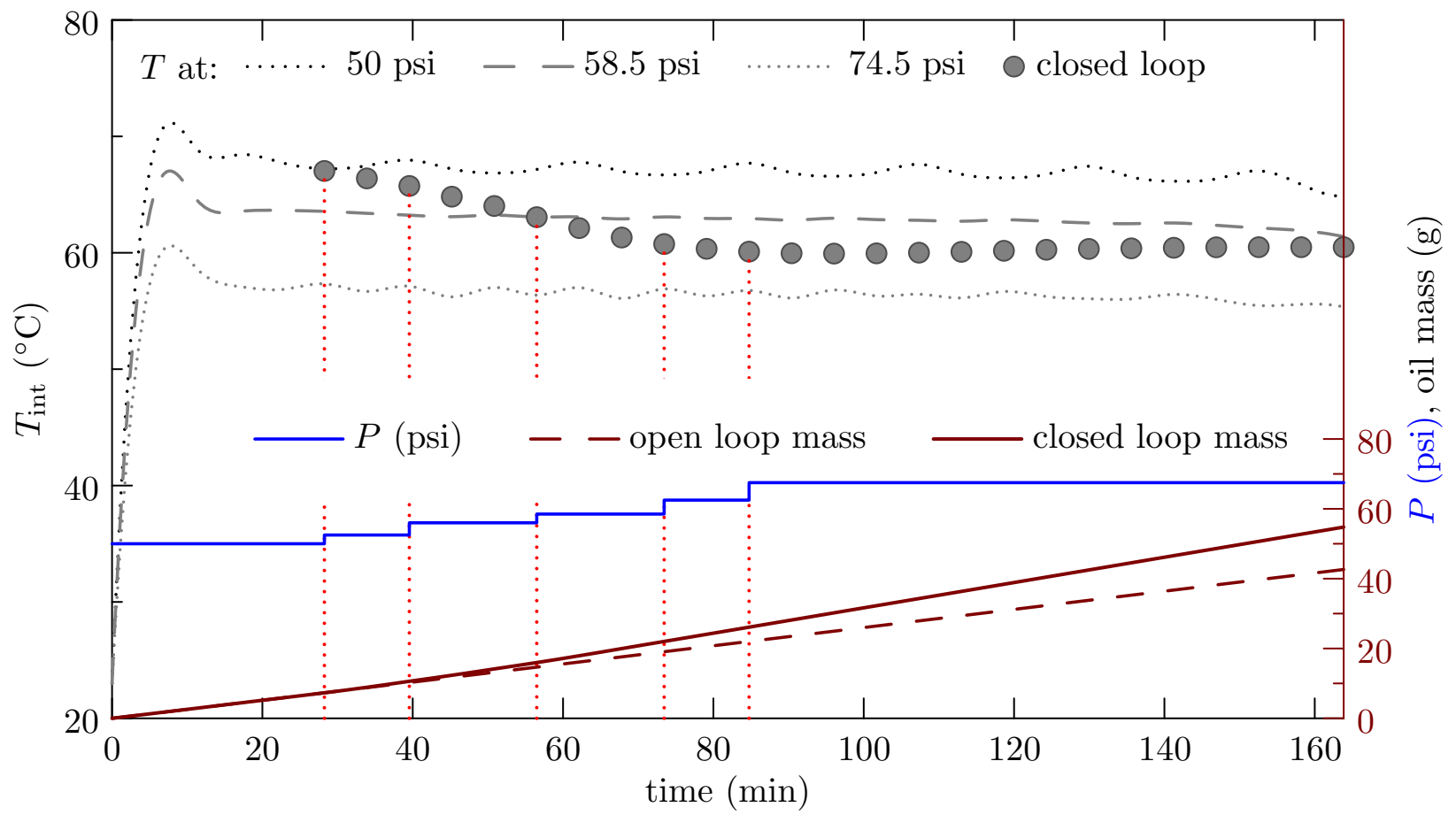

Figure 4.11: Closed Loop Result: Multiple upsets.

Thus, not only optimal policies along with objective functional values, but also state properties get navigated towards the desired optimal trajectories at the tweaked pressure. This phenomenon is showcased in Figure 4.12. Moreover, calculated squared errors at all the grid points corroborates the aforementioned observation in Figure 4.13 as we see the squared deviation between the nominal state and the adjusted state approaches zero upon control regulation. With the same processor configuration (as mentioned in section 4.4.1), this entire interpolation mechanism and the necessary control adjustment action concludes the simulation (over the span of $163.85 \mathrm{~min}$ ) in about 0.9 ps. It may be noted that the repository, which was determined and stored offline, required a total space of just 0.185 MB. Therefore, we can conclude that the state feedback proportional gain control action is potential enough for fast amendments of the desired nominal trends at new conditions which eradicates any chances of loss in the process output. 


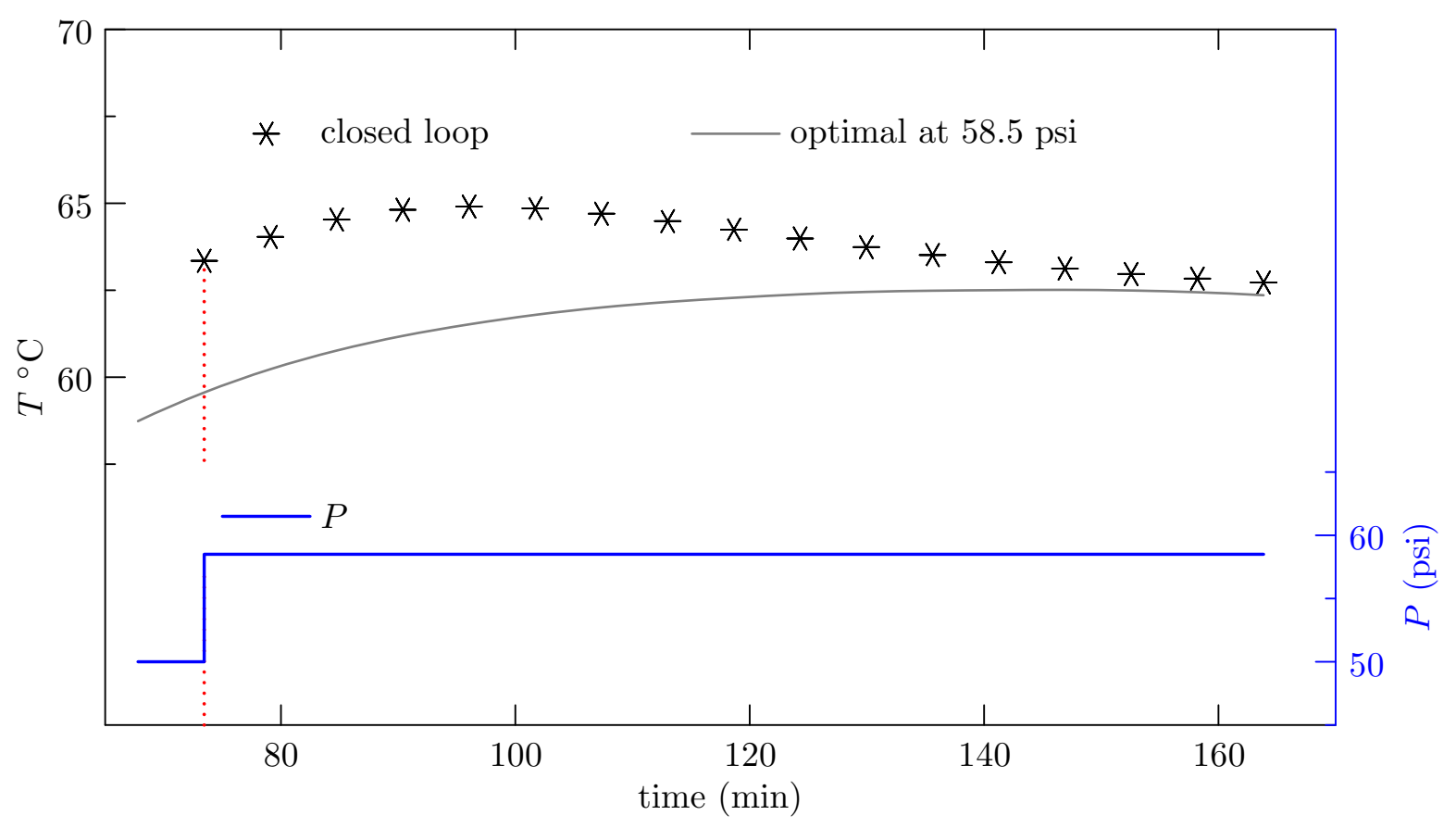

Figure 4.12: Optimal temperature at grid points $i=2$ and $j=3$.

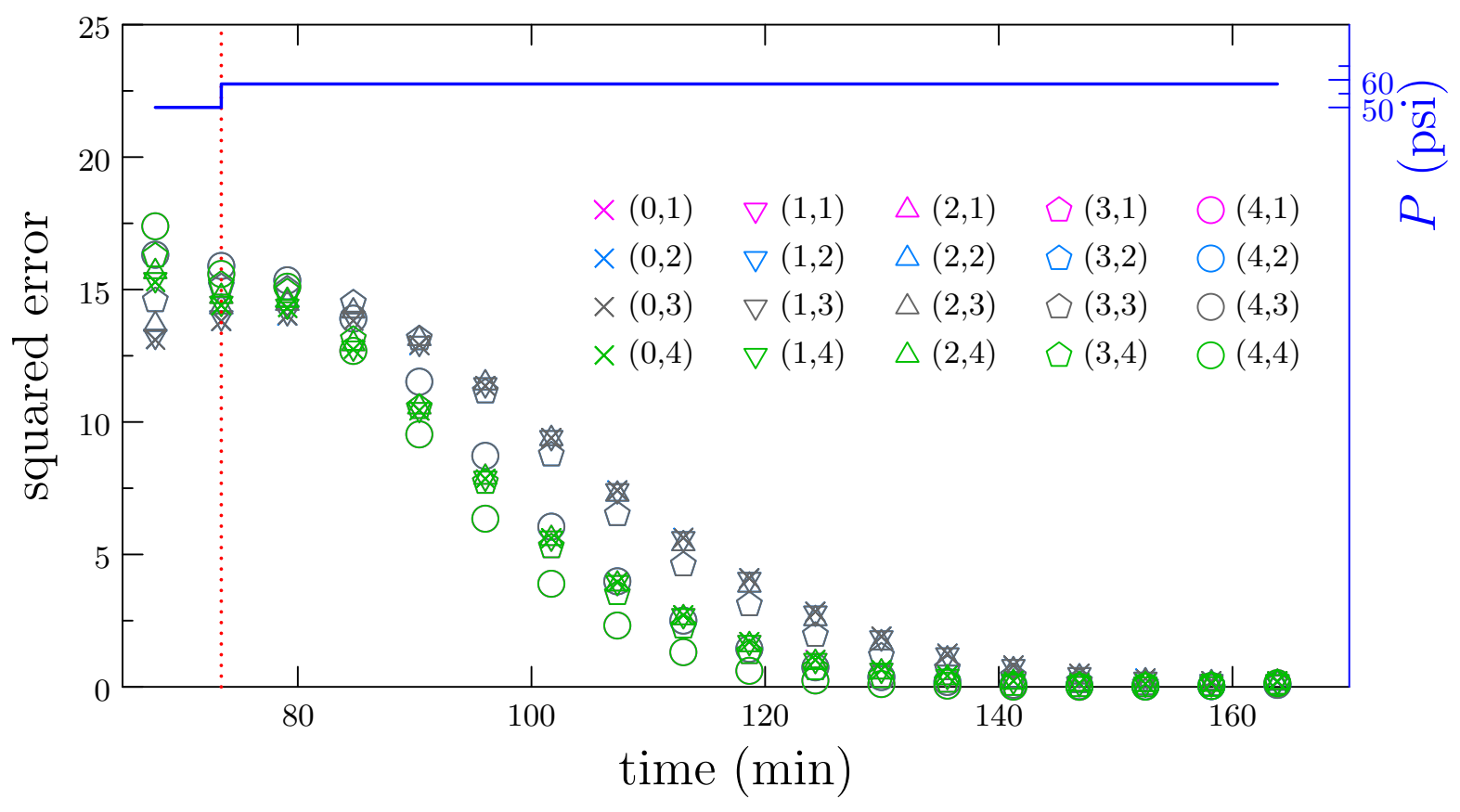

Figure 4.13: Error Analysis at Different Grid Points. 


\section{Chapter 5}

\section{Conclusions}

Initially, an optimal control framework was developed for nitrogen injection enhanced oil recovery using interfacial temperature versus time as a control function. The necessary conditions for maximum oil production were derived based on a distributed-parameter process model with a moving boundary. Then we derived system of Riccati equations and proportional gain feedback control law where adjustment to control was explicitly related to perturbed state with the aid of time-dependent gain parameter matrix. A computational algorithm was developed and programmed to determine the optimal control functions (using $\mathrm{C}++$ programming language), optimal gains, and optimal states at different pressure conditions, that is, $74.5 \mathrm{psi}, 58.5 \mathrm{psi}, 50 \mathrm{psi}, 43.5 \mathrm{psi}$ and $24.5 \mathrm{psi}$. This repository was utilized for interpolating (using an adjustment class coded in $\mathrm{C}++$ programming language) optimal conditions in the real time mode whenever there was a pressure upset.

Based on the interpolated data sets, the control action adjusted itself to steer the process to optimality at new prevailing pressure. This control adjustment mechanism (based on interpolating with respect to known process conditions) was not only adjusting the control function, but also predicting objective functional value (i.e., heavy oil mass) almost accurately at tweaked pressure. Moreover, sufficient adjustment time was mandated to maximize production at new boundary condition. Secondly, state properties across the physical model got updated and upon adjustment, variation with respect to the optimal state got substantially minimized as time proceeded. Overall, proportional gain control action is po- 
tential enough to track optimality whenever there is a pressure upset and maximize nitrogen injection recovery of heavy oil.

\subsection{Future Work}

There are numerous prospects that can be analysed on the basis of this control strategy:

1. We can apply NMPC (non-linear model predictive control) on the same distributed parameter system and compare the performance with our control methodology.

2. We can analyze for white noise and response delays for the same system and the process.

3. Like the proportional controller, we can extend the study to build a proportionalintegral controller and compare the performance. 
Appendices 


\section{Appendix A}

\section{Experimental Details}

Here, we describe various details of the experimental apparatus and equipment which is related to the experimental process described in Chapter 4, Section 4.1 .

\section{A.1 Apparatus for Experimental Investigation}

This section congregates list of various apparatus and sets of laboratory equipment which were employed to carry out this experimental investigation using nitrogen as the solvent for the recovery of heavy oil ([54]). These sets of equipment are:

1. The major equipment is a cylindrical pressure vessel with specified dimensions (internal diameter of $15 \mathrm{~cm}$ and a height of $80 \mathrm{~cm}$ ).

2. A digital thermometer (VWR traceable digital thermometer with recorder output, Mississauga, Ontario, Canada) is used for recording the changes in temperature of the pressure vessel.

3. A pressure transducer (Voltage Output Pressure Transducer, PX01C1-200G5T, Omega Inc., Canada) is employed in order to measure magnitude of nitrogen pressure inside the vessel.

4. There is a resistance temperature detector (RTD) (Rugged Transition Joint Probe, Newport Electronics, CA, US) which is used to measure the temperature of nitrogen 
inside the vessel.

5. A load cell (Miniature Load Cell Model 31 and Mid-Range Precision Miniature Load Cell model 31, Honeywell Sensotec, OH, US) is implemented within the system for tracking the diminution of mass of the physical model with respect to time as oil is being produced.

6. A flow-meter (In-line flow meters, FL46302, Omega Inc., QC, Canada) is also installed for the purpose of assisting in navigation of the nitrogen flow rate into the pressure vessel.

7. There are two pressure control valves present (PV101-10V, Omega Inc., QC, Canada) which are responsible for maintaining desired nitrogen pressure level inside the pressure vessel.

8. There are two heating tapes (Heat tape with the controller, HTWC 101, Omega Inc., MA, US) that are implemented to aid with the heating of nitrogen inside the pressure vessel.

9. A viscometer is employed (VISCO PRO 2000, Cambridge Viscosity, Inc., MA, US) to collect the recovered 'live oil' present in the collection tube as well as to determine the viscosity of the oil.

10. A steel flash tank of $300 \mathrm{~cm}^{3}$ capacity is used for collecting the 'live oil' and segregating nitrogen from it.

11. A physical reservoir model $(2.75 \mathrm{~cm}$ radius and $35 \mathrm{~cm}$ length $)$ is prepared from Fort Kent heavy oil (supplied by Saskatchewan Research Council, Alberta, Canada) of viscosity value of $1400 \mathrm{~g} \mathrm{~cm}^{-1} \mathrm{~min}^{-1}$ at approximately $25^{\circ} \mathrm{C}$ and glass beads (BALLOTINI Impact Beads, Potters Industries LLC, PA, US) are packed together inside the cylindrical stainless steel wire mesh. 
The mixture of oil with the glass beads are packed into a cylindrical stainless steel wire mesh. The model is homogeneous with a porosity of 0.38 and of permeability value of 4 Darcy (calculation showed in Appendix B). All the apparatus and sets of equipment listed above were connected to a computer (user interface) via a data acquisition system where the experimental/process changes were duly recorded using LabVIEW version 7.1 software application.

\section{A.2 Description of Various Parts}

In this section, various parts of the lab-scale experimental setup is described.

\section{The Measuring System}

A measurement system was employed for the process of associating numbers with physical quantities, phenomena or process variables. The mass of the specific apparatus used in the various steps were recorded and validated by using a measurement device known as a mass balance scale. It used to consist of a display for portraying mass (measured in unit of kilogram) of various experimental components like glass beads, cylindrical mesh, collection pot for heavy oil etc. Besides, it also had a rectangular platform to accommodate experimental components whose mass needed to be measured.

\section{Hot Air Oven}

Hot air ovens are electrical devices that operate by a mechanism known as dry heat sterilization. The oven is heated up to a desired set point level in terms of temperature and hot air is circulated throughout the internal core for maintaining that specific constant temperature level inside the oven. The transmission of heat is governed by the phenomena of forced convection. The power button aided to switch on the system (the hot air oven) and the setting knob helped in adjusting the temperature at its desired level. For each single run the lower limit of the temperature value was $23^{\circ} \mathrm{C}$ and it was raised to $70^{\circ} \mathrm{C}$. The same rise in temperature used to occur within $30 \mathrm{~min}$ and the system (inner core of the hot air 
oven) was maintained at this desired level during the heating operation. Inner core of the hot air oven consists a platform for supporting the control volume (holding pot for heavy oil mainly) during the heating operation. The circulation of air inside the system used to start as soon as the power button was switched on. Air was passed through the holes inside the core (along the vertical and horizontal walls) and this constant circulation of air aided in proper temperature regulation.

\section{The Physical Model}

The experiment started with the preparation of the physical model using heavy oil. Physical model is a control volume analogous to the exact geological core comprising heavy oil or bitumen packed with sand materials.

The first step was to weigh the pot for measuring and recording the mass of heavy oil. The recorded weight of the empty pot was $0.54 \mathrm{~kg}$. Heavy oil was then poured into the pot from the supply of oil (in the laboratory, the heavy oil was stored in a 55-gallon drum) which displayed an increment in the mass to $2.54 \mathrm{~kg}$. This weight was recorded and then the pot containing heavy oil was placed inside the hot air oven and was heated for 30 min at a constant temperature magnitude of $70^{\circ} \mathrm{C}$; this operating condition (in terms of temperature) aided in the reduction of the viscosity of heavy oil that was mandated not only for the flow but also for proper mixing of the same with the glass beads. The weighing scale was once again brought back to zero datum level and a cylindrical mesh of known dimension was placed on the rectangular platform and the weight was recorded as $0.08 \mathrm{~kg}$. The mesh was filled with $3.78 \mathrm{~kg}$ of the glass beads and in the following step the same amount of glass beads was mixed with the preheated heavy oil. The glass beads were then added gradually (with continuous stirring or mixing operation) to the heated controlled volume (heavy oil) and this strategy was applied in order to ensure proper mixing of the glass beads with heavy oil without trapping of air bubbles. This gradual mixing technique maintained homogeneity of the mixture. Once the same was properly mixed, it was left to settle overnight for future experimental procedures. 


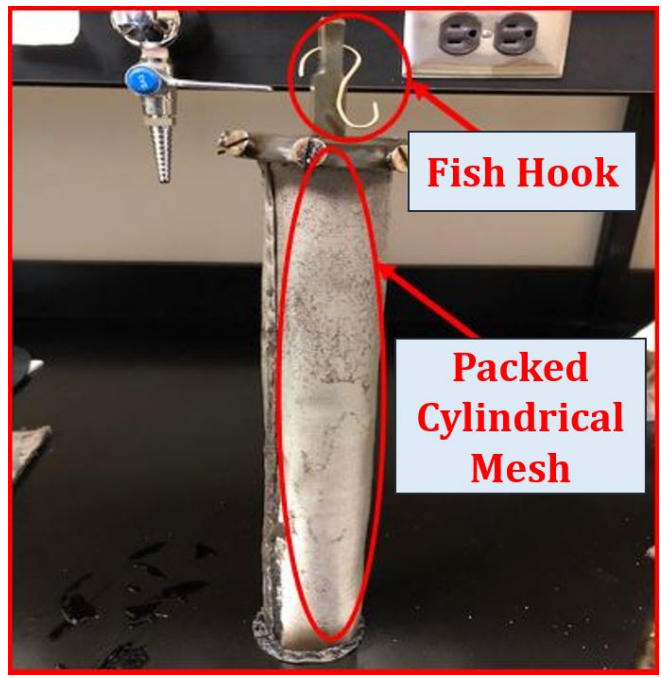

Figure A.1: Heavy oil filled mesh

The saturated mixture was poured slowly into the cylindrical wire mesh as a thin layer. It was left to settle under gravity for $5 \mathrm{~min}$; it was then refilled with additional layers of heavy oil. This procedure was repeated until the cylindrical mesh was filled to its brim; once the system was thoroughly packed with heavy oil-glass beads mixture, it was weighed and the mass was recorded $1.28 \mathrm{~kg}$. The packed cylindrical model was secured tightly by tightening screws on the top of the mesh and the same was placed inside the pressure vessel with the help of a bent stainless steel fish hook (Figure A.1).

\section{Cylindrical Vessel}

A visual representation of the cylindrical vessel utilized in the experiment is illustrated in Figure A.2. Temperature was navigated with the aid of heating tapes that emerged from the heating source and coolant flow line. On the other hand, pressure was injected as well as regulated with nitrogen flow line, pressure transducer and pressure gauge. Various measuring instruments like rotameter and pressure gauge were attached at the entry of the pressure vessel in conjunction with coolant flow line and nitrogen flow line respectively.

The heating system: The heating supply devices controlled the supply of heat to the system. These 2 heat supply devices were responsible for the central heating of the interior 
core of the cylindrical pressure vessel and they were usually adjusted depending on the system requirement in terms of higher or lower temperature value while conducting different experimental runs.

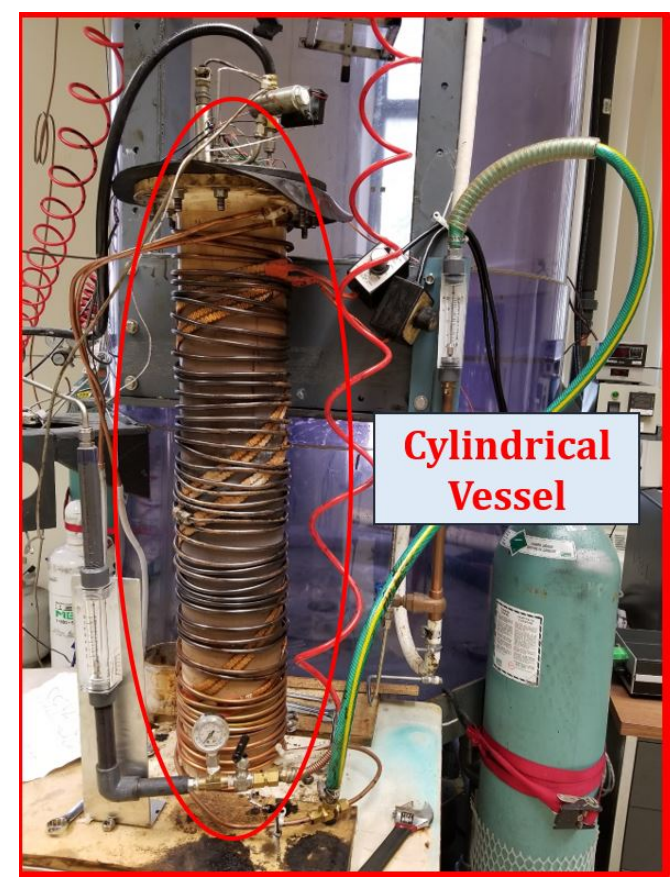

Figure A.2: Cylindrical Pressure Vessel with Coolant Line

The devices were calibrated in terms of percentage of the supply heat wattage and the specification of the same was 120 Volts-288 Watts. They were directly connected to a Wattmeter (energy meter) for the display as well as the regulation of different electrical parameters (Wattage, Voltage and Ampere).

The coolant system: The copper coils surrounding the pressure vessel were responsible for maintaining the temperature within the system. In this case, the coolant was cold water at an ambient temperature of $15^{\circ} \mathrm{C}$. The rotameter was connected to the system via a green hose line and the rotameter valve (directly below the rotameter) was responsible for controlling the coolant flow rate. The rotameter was calibrated as per the scale of 0-2 gpm and the same could be regulated accordingly with the aid of the valve. Rotameter was responsible for measuring the volumetric flowrate of the water used in the experiment in order to cool 
down the pressure vessel whenever the system experienced unwanted elevated temperature levels. The valve which controls the supply of water via flowrate adjustment is seen below the rotameter in the picture. The flow mechanism was from downward to upward direction against gravitational force allowing effective accumulation of the coolant around the vessel in order to cool down the system by enhancing effective heat transfer area and eradicating the possibilities of dry spots along the direction of the flow. Usually, the coolant system was not in use; it was used only in case of moderate adjustment of desired temperature level.

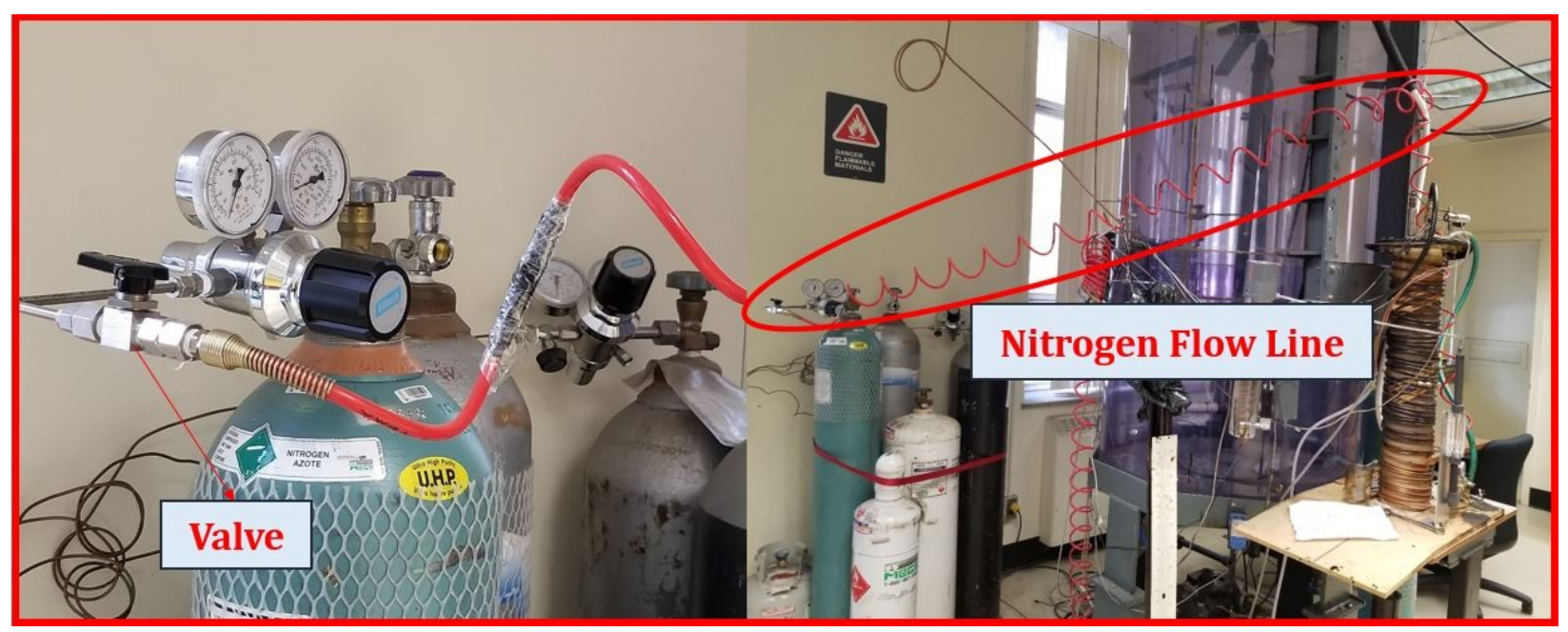

Figure A.3: Nitrogen Flow Line

Nitrogen pressure regulation system: In the diagram (Figure A.3), the red line is representative of the pathway of nitrogen that was taken inside the cylindrical vessel from the source. The pressure gauge with a valve was employed to control the magnitude of nitrogen pressure entering the system. A solenoid valve at the top of the cylindrical vessel regulate the opening of the fluid (nitrogen in this case) flow in the valve. This is basically an electro-mechanical device where the solenoid utilizes an electric current in order to generate a magnetic field, hence operating a mechanism which is used for flow regulation. A differential pressure transducer was used to measure the pressure difference across the porous media as nitrogen was passed throughout the system. Both solenoid valve and the pressure transducer was attached at the top of the cylinder. It had to be properly screwed with the 
help of tightening screws, washers and bolts in order to ensure minimal (or, zero) leakage that could hinder the accuracy of the experimental results. Additionally, in our experimental procedures pressure was controlled and tightly regulated with the help of a set point fine-tuner in the LabVIEW Interface; the same was used in conjunction with the solenoid valve for accurate experimental results.

\section{Load Cell and Collection Tube}

The heavy oil collected from the pressure vessel was directed towards the collection tube and a load cell was attached to it. A digital thermometer upstream of the collection tube aided in monitoring and recording of the temperature in the pressure vessel. Figure A.4 displays the load cell at the bottom of the pressure vessel which tracked the accumulated mass of heavy oil with time. The mass of the oil was one of the desired outputs that was being observed while conducting the experiments; therefore, this was one of those cases where the load cell played a vital role in tracking the altercation in mass. The collection tube had to be flushed (with Varsol) at the onset of each experimental trials in order to ensure that the system was totally free of oil (that may have been left back in the tube from previous experiments).

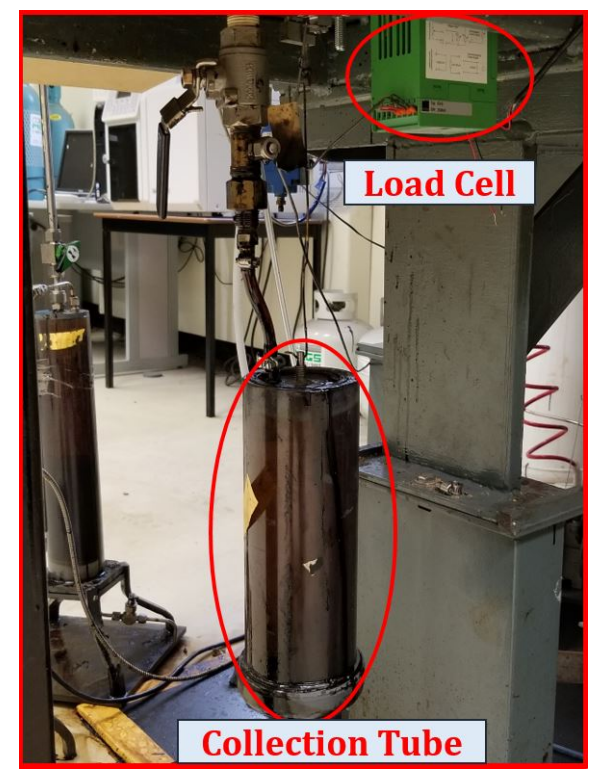

Figure A.4: Load Cell and Collection Tube 


\section{Viscometer}

From the collection tube live heavy oil (heavy oil with nitrogen dissolved in it) was directed to a viscometer shown in Figure A.5 which was responsible for providing the magnitude of viscosity (depending on operating temperature) of the oil. The viscosity value was directly displayed in the control panel of the LabVIEW with the aid of the data acquisition system.

\section{Flash Tank}

In Figure A.5, the flash tank was responsible for the transition from live oil (heavy oil with dissolved nitrogen) to dead oil (pure heavy oil when dissolved nitrogen was flushed out of it) by separating the dissolved nitrogen from the oil as it passed through this system. The flash tank is basically a cylindro-conical vessel used to lower the pressure on lighter fraction of the mixture and helps it to degas, or flash out, hence separating it from remaining heavier fraction. This is the process occurring within the system between the transitions of oil from the live oil to dead oil phase where the heavier dead oil was collected in a collection pot at the bottom of the flash tank.

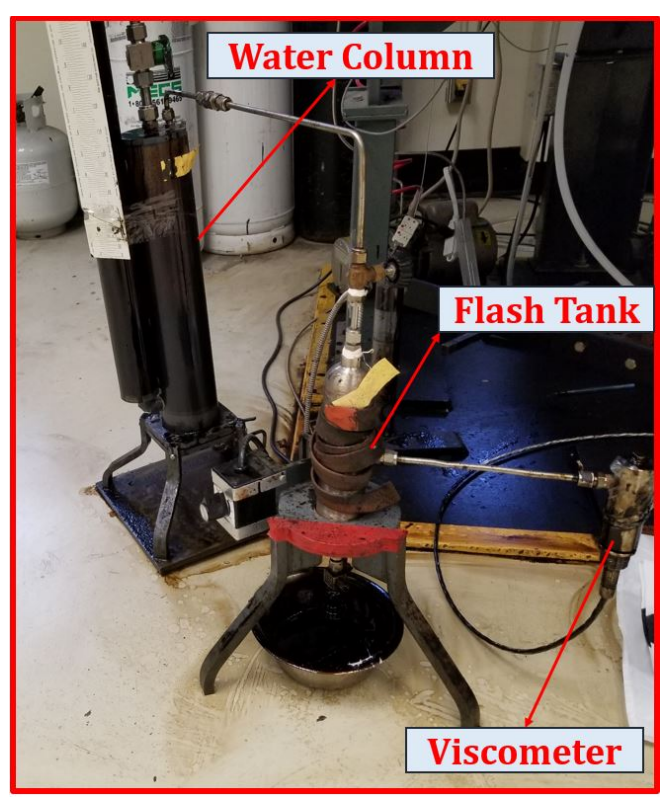

Figure A.5: Viscometer, Flash Tank and Water Column 


\section{Water Columns}

The water column located adjacent to the flash tank in Figure A.5; the scale allowed the investigators to observe and track the difference in volume after the separation of the nitrogen from oil inside the flash tank. The differential change in height multiplied with the cross sectional area of the column aided to get the amount of dissolved nitrogen in live oil.

\section{Data Acquisition System}

Data acquisition dealt with the process of sampling signals (that measured real world physical conditions and converted the same into digital numeric values) of various tangible or intangible manipulated or regulated physical phenomena. Figure 4.1 displays the connection wires of the apparatus to the acquisition system which was in turn connected with the computer where the process variables were recorded using the control panel display of LabVIEW 7.1 software application.

\section{Cleaning Station}

This is the area that was employed mainly for cleaning purposes. Varsol was the cleaner (liquid) that was used which is a premium quality low odour paint thinner with a medium to fast evaporation rate that acted as a versatile solvent for degreasing sets of equipment. Different apparatus being used were cleaned before and after the experimental setup with this chemical in the area displayed below. The red station was filled with this chemical and all the cleaning operations were performed inside this closed system.

\section{A.3 Determination of Interfacial Concentration}

At the onset of each set of experiment, atmospheric air was purged from the pressure vessel by applying vacuum close to 14.5 psi-absolute using a vacuum pump. Then, the pressure vessel was filled with $25 \mathrm{~g}$ of heavy oil. The filling up was done slowly and layer by layer in order to avoid entrapment of air within heavy oil layers. After thoroughly sealing the 
pressure vessel, it was placed in a water bath (as shown in Figure A.6).

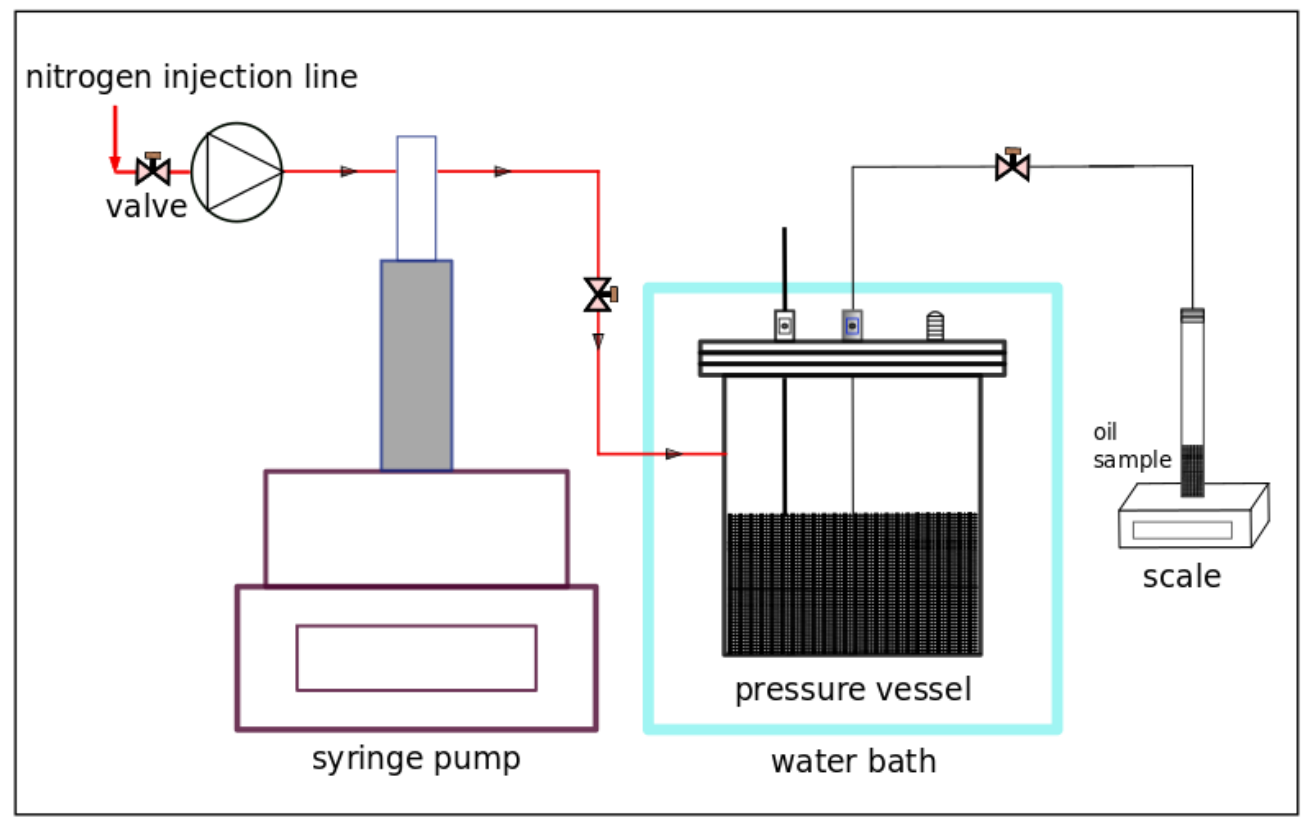

Figure A.6: Schematic diagram of nitrogen-heavy oil system for solubility measurement

The objective of the heater tapes and the water jacket was to maintain the temperature of the pressure vessel at the desired set points. The top end of the vessel was connected to the valve regulated nitrogen inflow line and the desired pressure conditions were regulated by LabVIEW block diagram. The accumulated mass of oil was weighed and then it was heated inside the hot air oven at around $70^{\circ} \mathrm{C}$ in order to release dissolved and residual nitrogen in the oil. After flashing the solvent gas, the dead oil was weighted and from mass balance, nitrogen mass fraction in the oil was calculated. The run time for each test was 430 min.

Interfacial Concentration of nitrogen at different temperatures and pressures was derived using following mass balance equation

$$
w_{\mathrm{int}}=\frac{m_{\mathrm{gas}}}{m_{\mathrm{gas}}+m_{\mathrm{oil}}}
$$

For example, at 43.5 psi and $25^{\circ} \mathrm{C}$ temperature, weight of oil (after heating) was found to be $2.46 \mathrm{~g}$ and weight of oil (before heating) was $2.4851 \mathrm{~g}$. Hence, amount of dissolved nitrogen 
was $0.0251 \mathrm{gm}$. Therefore, from mass balance, interfacial concentration under prevailing condition was calculated to be:

$$
w_{\text {int }}=\frac{0.0251}{0.0251+2.46}=0.0101
$$

Different operating pressure magnitudes were selected to be $74.5 \mathrm{psi}, 58.5 \mathrm{psi}, 50 \mathrm{psi}, 43.5 \mathrm{psi}$ and 24.5 psi respectively. For each of these pressure magnitudes, experiments were performed at different values of temperature namely $25^{\circ} \mathrm{C}, 50^{\circ} \mathrm{C}, 75^{\circ} \mathrm{C}$ and $90^{\circ} \mathrm{C}$ respectively. Thereafter, from mass balance, we obtained the dependencies of solubility of nitrogen on pressure and temperature. Results are discussed in Appendix D 


\section{Appendix B}

\section{Permeability Calculation}

Permeability $(K)$ was calculated using the following equation:

$$
K=\frac{P_{\mathrm{o}} \times Q_{\mathrm{o}} \times \mu_{\text {nitrogen }} \times L}{\left(\frac{\pi D^{2}}{4}\right) \times P_{\mathrm{m}} \times \triangle P} .
$$

where $P_{\mathrm{i}}$ and $P_{\mathrm{o}}$ are the pressure at inlet port and outlet ports of the cylindrical vessel respectively, $\triangle P$ is the pressure difference, $P_{\mathrm{m}}$ is mean pressure, $L$ is length of the cylindrical vessel, $\mu_{\text {nitrogen }}$ is nitrogen viscosity at room temperature, $Q_{\mathrm{o}}$ is flow rate of nitrogen and $D$ is the diameter of the cylindrical vessel. Using following data, value of $K$ is 4 Darcy.

\begin{tabular}{|c|c|}
\hline Parameter & Value \\
\hline$P_{\mathrm{o}}(\mathrm{atm})$ & 1.153 \\
$P_{\mathrm{i}}(\mathrm{atm})$ & 1.142 \\
$\triangle P(\mathrm{~atm})$ & 0.011 \\
$Q_{\mathrm{o}}\left(\mathrm{cm}^{3} \mathrm{~s}^{-1}\right)$ & 1.7 \\
$\mu_{\text {nitrogen }}(\mathrm{cP})$ & 0.0175 \\
$P_{\mathrm{m}}(\mathrm{atm})$ & 1.1475 \\
$L(\mathrm{~cm})$ & 35 \\
$D(\mathrm{~cm})$ & 5.5 \\
\hline
\end{tabular}




\section{Appendix C}

\section{Viscosity and Diffusivity Data}

\section{C.1 Viscosity Data}

Figure $\mathrm{C} 1$ and Figure $\mathrm{C} 2$ describe viscosity of heavy oil at different temperatures and pressures.

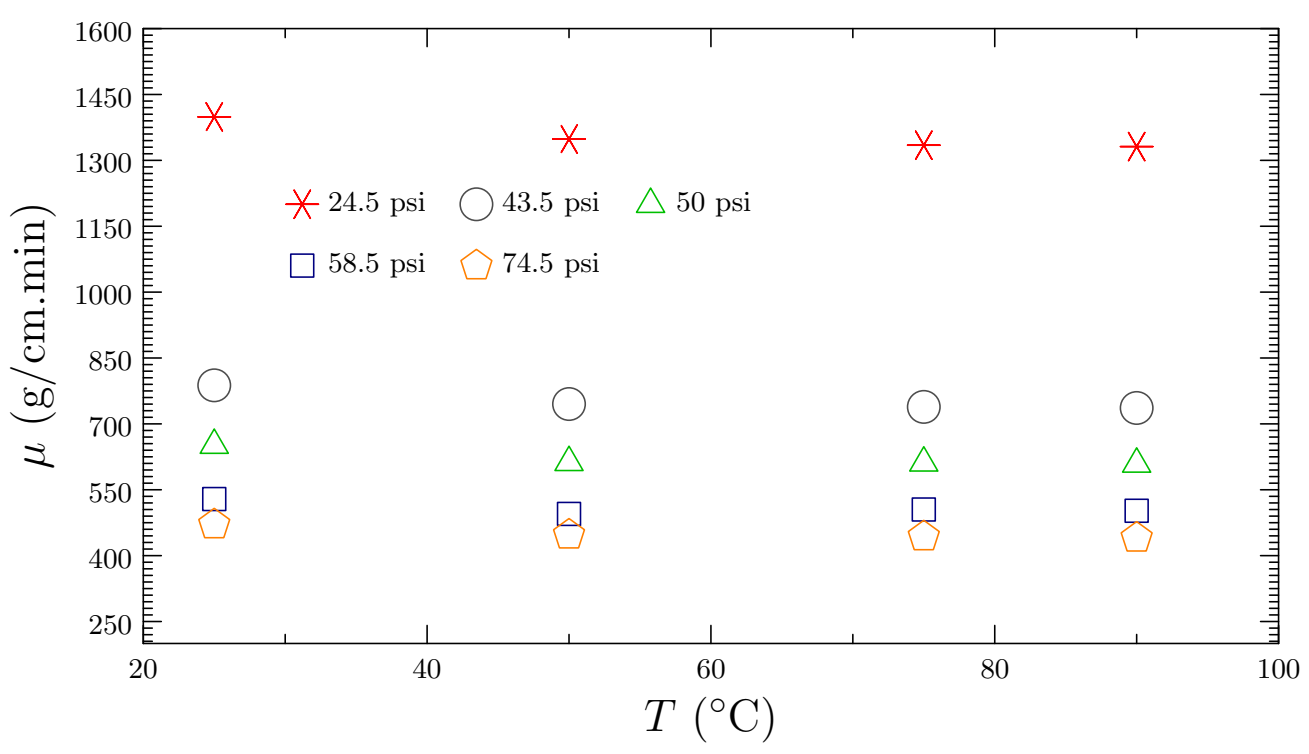

Figure C.1: Viscosity of Heavy Oil at Different Temperatures.

We can clearly see that viscosity remains almost constant in the range of temperature variation (Figure C1) but with increasing pressure, the viscosity magnitude drops (Figure $\mathrm{C} 2)$. 


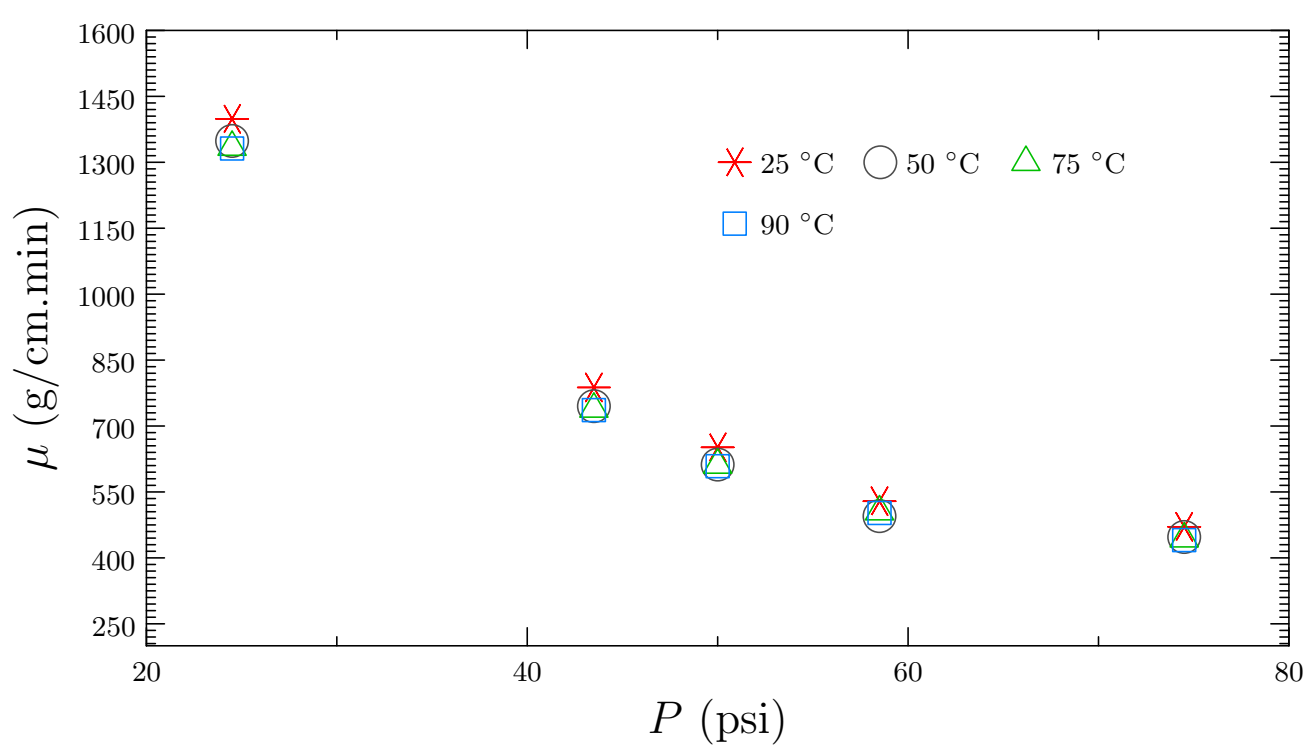

Figure C.2: Viscosity of Heavy Oil at Different Pressures.

Eventually, we can express pressure, concentration and temperature dependent heavy oil viscosity $(\mu)$ by the following correlation

$$
\mu=\left(a_{0}+\frac{a_{4}}{T}+a_{7} w\right) P^{2}+\left(a_{1}+\frac{a_{5}}{T}+a_{8} w\right) P+a_{2}+\frac{a_{6}}{T}+a_{9} w .
$$

In the above correlation, $a_{0}, a_{1}, a_{2}, a_{4}, a_{5}, a_{6}, a_{7}, a_{7}, a_{8}$, and $a_{9}$ are constant parameters in their corresponding units as shown in Table C.1.

\section{C.2 Diffusivity Data}

At the onset of our simulation studies, we evaluate diffusivity $D(w)$ of nitrogen as a function of solvent mass fraction using a method developed elsewhere [53, 55]. In the aforementioned algorithm, we deploy the results in terms of experimental mass at different pressure $(P)$ and temperature $(T)$ as our initial guess. Eventually we obtain the influence of concentration, pressure and temperature on diffusivity. Figure C.3 shows that the diffusivity increases with the solvent mass fraction to a maximum and then drops subsequently.

These diffusivity data at different pressure is further deployed in the model simulation, open loop simulation and eventually adjusted in closed loop simulation. 
Table C.1: Viscosity Parameters

\begin{tabular}{|c|c|c|}
\hline Parameters & Unit & Values \\
\hline$a_{0}$ & $\mathrm{~g} \mathrm{~cm}^{-1} \min ^{-1} \mathrm{psi}^{-2}$ & 0.4379 \\
\hline$a_{1}$ & $\mathrm{~g} \mathrm{~cm}^{-1} \min ^{-1} \mathrm{psi}^{-1}$ & -61.194 \\
\hline$a_{2}$ & $\mathrm{~g} \mathrm{~cm}^{-1} \min ^{-1}$ & 2533.9 \\
\hline$a_{4}$ & $\mathrm{~g}^{\circ} \mathrm{C} \mathrm{cm}^{-1} \mathrm{~min}^{-1} \mathrm{psi}^{-2}$ & -0.0496 \\
\hline$a_{5}$ & $\mathrm{~g}^{\circ} \mathrm{C} \mathrm{cm}^{-1} \mathrm{~min}^{-1} \mathrm{psi}^{-1}$ & -16.168 \\
\hline$a_{6}$ & $\mathrm{~g}^{\circ} \mathrm{C} \mathrm{cm}^{-1} \mathrm{~min}^{-1}$ & 2950.3 \\
\hline$a_{7}$ & $\mathrm{~g} \mathrm{~cm}^{-1} \mathrm{~min}^{-1} \mathrm{psi}^{-2}$ & 0.1532 \\
\hline$a_{8}$ & $\mathrm{~g} \mathrm{~cm}^{-1} \mathrm{~min}^{-1} \mathrm{psi}^{-1}$ & -7.9564 \\
\hline$a_{9}$ & $\mathrm{~g} \mathrm{~cm}^{-1} \min ^{-1}$ & 160 \\
\hline
\end{tabular}




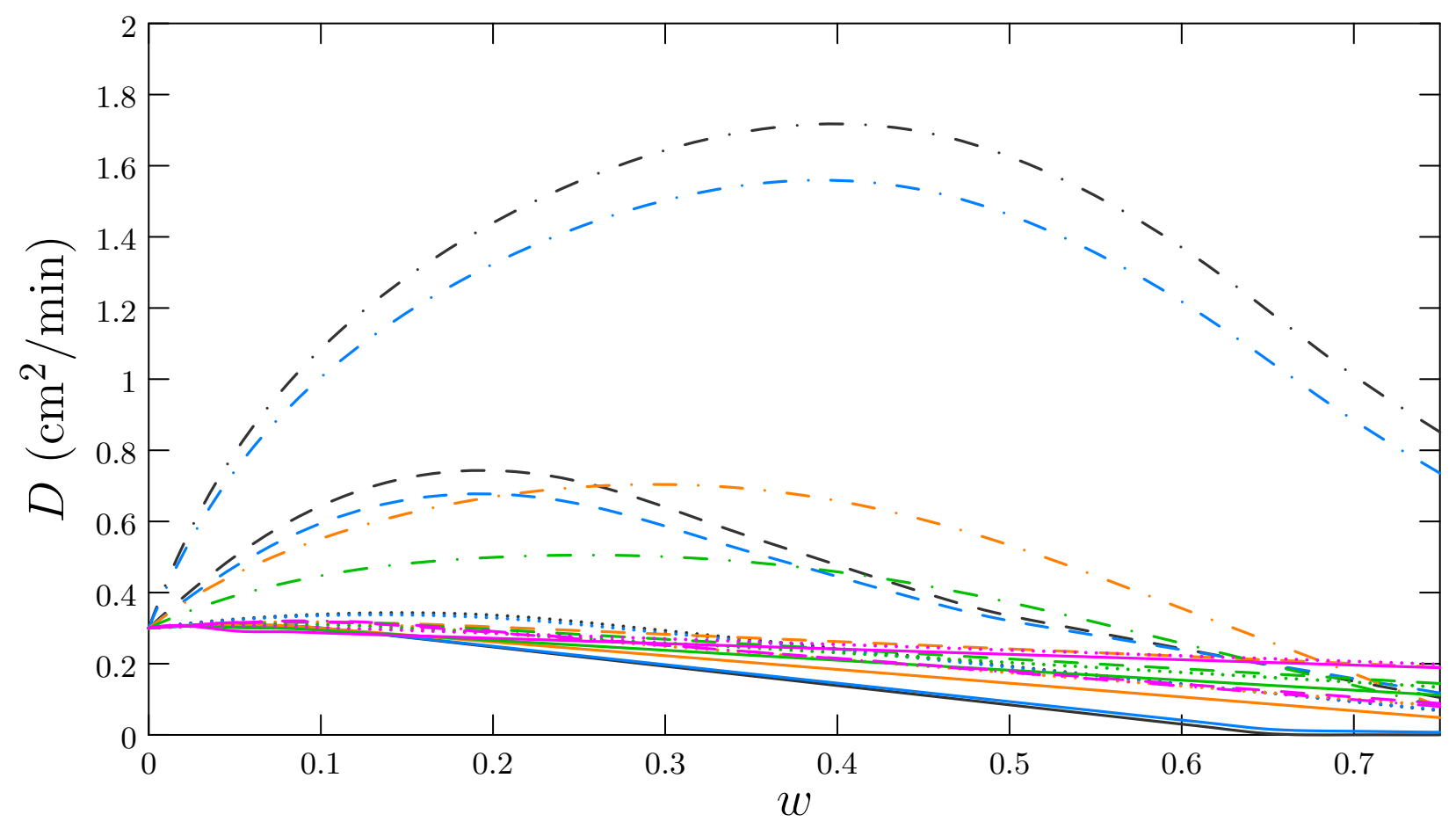

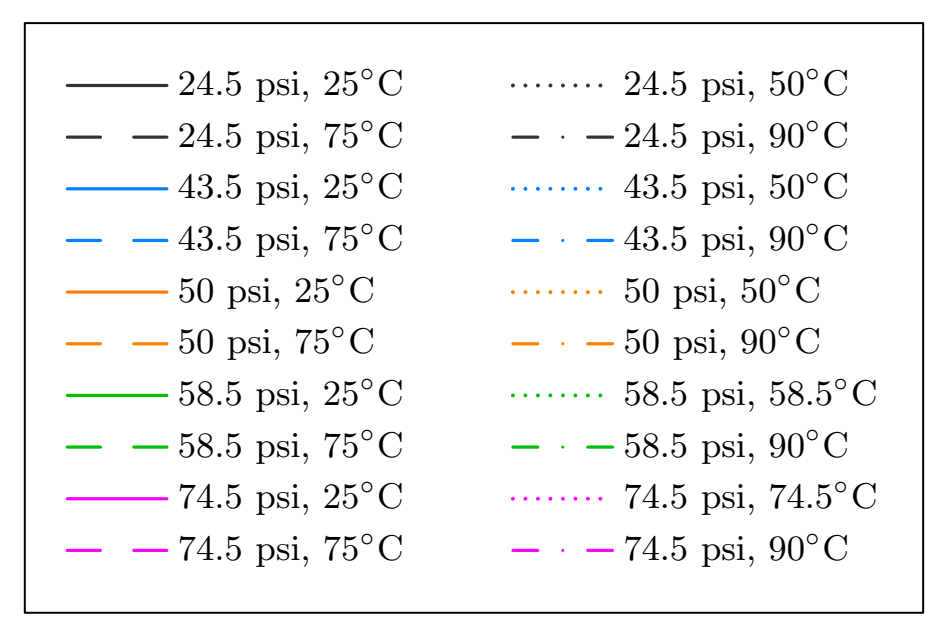

Figure C.3: Diffusivity versus concentration at different temperatures and pressures. 


\section{Appendix D}

\section{Experimental Results}

\section{D.1 Experimental Result - Solubility Data}

Figure D.1 depicts the dependencies of solubility of nitrogen on pressure and temperature and clearly we see that as temperature and pressure rises, solubility (interfacial concentration or, dissolution of nitrogen in heavy oil) increases.

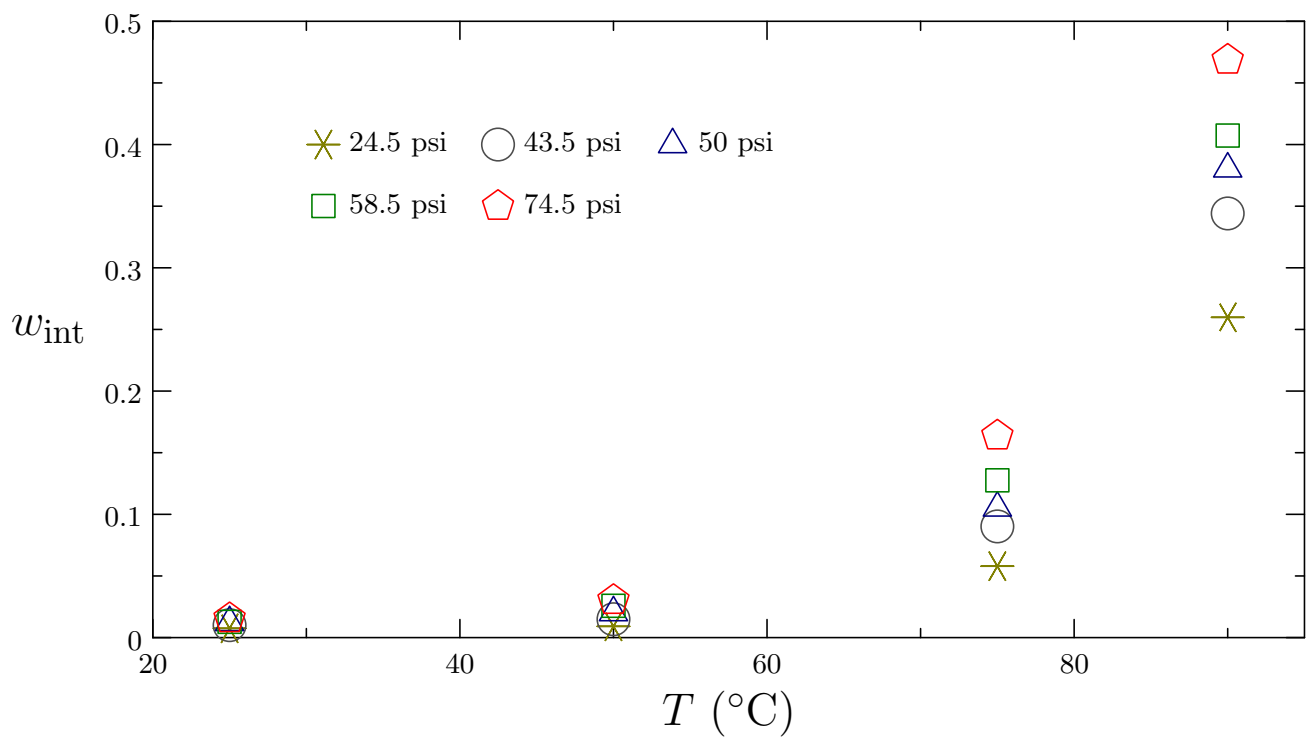

Figure D.1: Solubility at different temperatures and pressures.

The obtained data points furnish the boundary conditions of the mass transfer model under multifarious pressure conditions. 


\section{D.2 Experimental Result - Heavy Oil Mass}

The state-of-the-art experimental procedure described in Chapter 4, Section 4.1 yields the following results of heavy oil recovery.

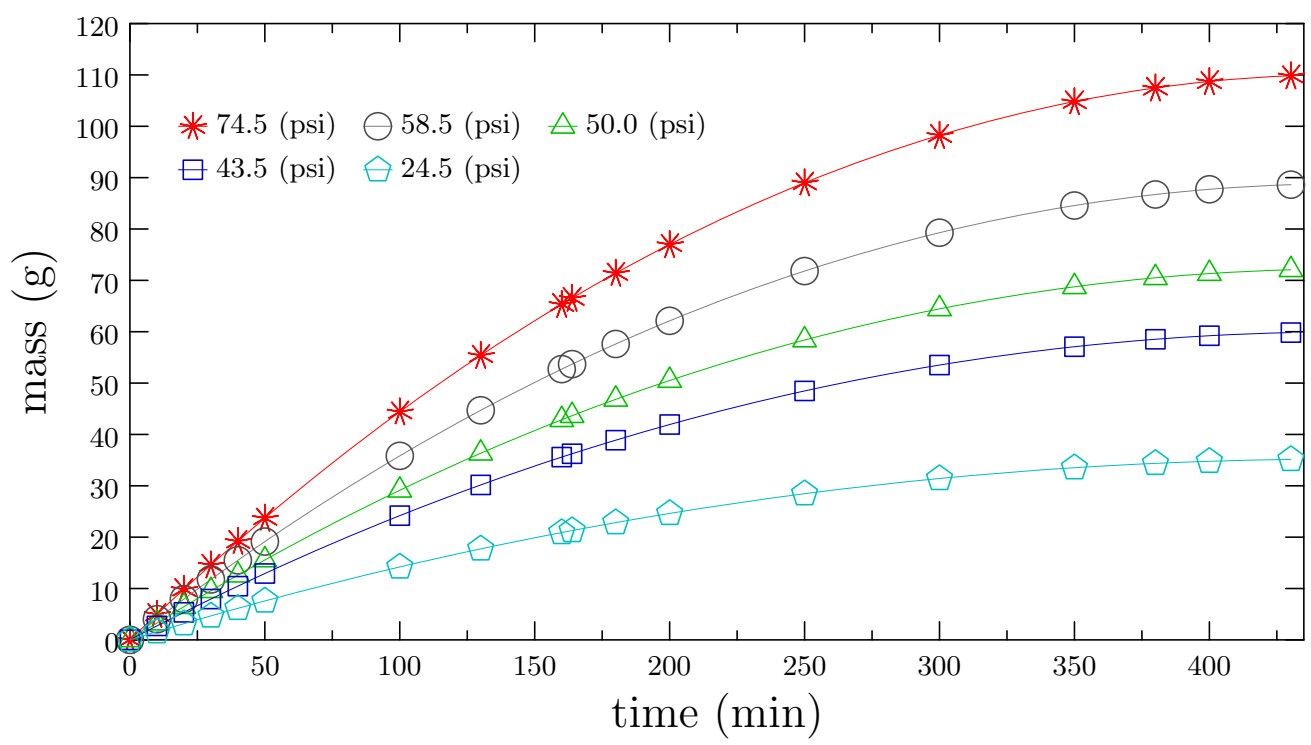

Figure D.2: Experimental mass at different pressures at $90^{\circ} \mathrm{C}$.

Figure D.2 describes amount of heavy oil recovery for a span of $430 \mathrm{~min}$ at $90^{\circ} \mathrm{C}$. It is clearly seen that mass gets saturated towards the end of the process at any pressure. Moreover, at a fixed temperature $\left(90^{\circ} \mathrm{C}\right.$ ), as pressure rises (from 24.5 psi to $74.5 \mathrm{psi}$ ), amount of heavy oil recovered gets increased (from $35.1 \mathrm{~g}$ to $109.8 \mathrm{~g}$ ) as well. After $163.85 \mathrm{~min}$, amount of heavy oil recovered was $21.3 \mathrm{~g}, 36.2 \mathrm{~g}, 43.7 \mathrm{~g}, 53.7 \mathrm{~g}$ and $66.5 \mathrm{~g}$ at $24.5 \mathrm{psi}, 43.5 \mathrm{psi}, 50 \mathrm{psi}$, $58.5 \mathrm{psi}$ and $74.5 \mathrm{psi}$, respectively.

Figure D.3 portrays that at a given pressure (74.5 psi), as temperature rises from $25^{\circ} \mathrm{C}$ to $90{ }^{\circ} \mathrm{C}$, we obtain more heavy oil. Also, towards $430 \mathrm{~min}$, mass recovery gets saturated as earlier. These observations concur with the experimental data investigated by Booran et al. ([54]). Thus, we conclude:

1. At any given pressure, as temperature rises, oil recovery increases. 
2. At any given temperature, as pressure increases, oil recovery gets enhanced.

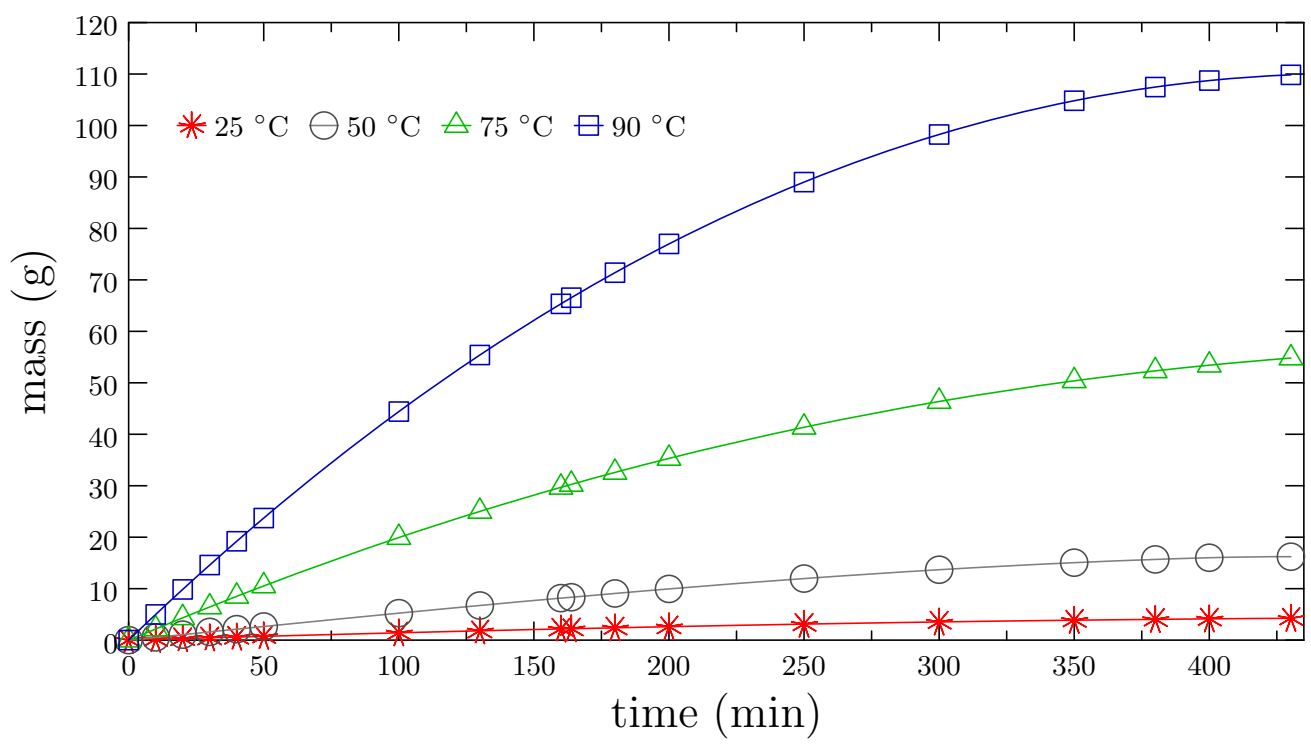

Figure D.3: Experimental mass at different temperatures at 74.5 psi.

In Chapter 4, Section 4.4 we utilize these experimental results for different simulation studies and comparisons in terms of objective functional. 


\section{Appendix E}

\section{Derivation of Process Model Equations}

\section{E.1 Mathematical Modeling for Mass Fraction}

Mass fraction of nitrogen is to be evaluated as this gas gets dissolved in to the heavy oil. Unsteady state differential mass balance equation for nitrogen is given by

$$
\frac{\mathrm{d}(\triangle V \phi \rho w)}{\mathrm{d} t}=(v \triangle A \rho w)_{z+\triangle z}-(v \triangle A \rho w)_{z}-(J \triangle S)_{r+\triangle r}+(J \triangle S)_{r}
$$

where, $\triangle V=2 \pi r \triangle r \Delta z$ is the differential volume, $\triangle S=2 \pi r \Delta z$ is the differential surface area along radial direction (transverse area with respect to diffusive flux) and $\triangle A=2 \pi r \Delta r$ is the differential surface area along axial direction (transverse area with respect to oil velocity) of the element respectively. Additionally, $\rho$ is oil density, $w$ is mass fraction of nitrogen in the medium and $\phi$ is medium porosity. $J$ represents diffusive mass flux of nitrogen along radial direction and this radial flux can be written as

$$
J=-\rho D \phi \frac{\partial w}{\partial r}
$$

where $D$ is undetermined pressure, concentration and temperature dependent diffusion coefficient of nitrogen in the porous medium (discussed in Appendix C, section C.2. . $v$ is assumed to be Darcy velocity of oil in the downward direction and is given by

$$
v=\frac{K_{\mathrm{r}} K \rho g}{\mu}
$$


where $K_{\mathrm{r}}$ and $K$ are the relative permeability and permeability of the porous medium respectively. Besides, $g$ is gravity and $\mu$ represents pressure, concentration and temperature dependent oil viscosity (correlation explained in Appendix C, section C.1). $T$ represents temperature and $P$ indicates pressure. Taking the limits of both $\triangle r$ and $\triangle z$ to zero ([56]), previous equations are moulded as the following

$$
\frac{\partial w}{\partial t}=\frac{1}{\phi} \frac{\partial(v w)}{\partial z}+\frac{1}{r} \frac{\partial}{\partial r}\left(\operatorname{Dr} \frac{\partial w}{\partial r}\right) .
$$

Further simplification yields

$$
\frac{\partial w}{\partial t}=\frac{\alpha}{\phi \mu} \frac{\partial w}{\partial z}+\frac{\alpha w}{\phi} \frac{\partial}{\partial z}\left(\frac{1}{\mu}\right)+\frac{D}{r} \frac{\partial w}{\partial r} \frac{\partial r}{\partial r}+\frac{D r}{r} \frac{\partial}{\partial r}\left(\frac{\partial w}{\partial r}\right)+\frac{r}{r} \frac{\partial w}{\partial r} \frac{\partial D}{\partial r} .
$$

We consider $\alpha=K_{\mathrm{r}} K \rho g$. Moreover, we evaluate the derivative by considering dependencies of viscosity and diffusivity on both temperature and concentration at a given operating pressure. Thus,

$$
\begin{aligned}
\frac{\partial w}{\partial t}= & \frac{\alpha}{\phi \mu} \frac{\partial w}{\partial z}-\frac{\alpha w}{\phi \mu^{2}}\left(\frac{\partial \mu}{\partial w} \frac{\partial w}{\partial z}+\frac{\partial \mu}{\partial T} \frac{\partial T}{\partial z}\right)+\frac{D}{r} \frac{\partial w}{\partial r}+D \frac{\partial^{2} w}{\partial r^{2}} \\
& +\frac{\partial w}{\partial r}\left(\frac{\partial D}{\partial w} \frac{\partial w}{\partial r}+\frac{\partial D}{\partial T} \frac{\partial T}{\partial r}\right) .
\end{aligned}
$$

Upon rigorous rearrangement, final form of the mass transfer model is

$$
\begin{aligned}
\frac{\partial w}{\partial t}= & \frac{D}{r} \frac{\partial w}{\partial r}+D \frac{\partial^{2} w}{\partial r^{2}}+\frac{\partial D}{\partial w}\left(\frac{\partial w}{\partial r}\right)^{2}+\frac{\partial D}{\partial T}\left(\frac{\partial w}{\partial r}\right)\left(\frac{\partial T}{\partial r}\right)+\frac{\alpha}{\phi \mu} \frac{\partial w}{\partial z} \\
& -\frac{\alpha w}{\phi \mu^{2}}\left(\frac{\partial \mu}{\partial w} \frac{\partial w}{\partial z}+\frac{\partial \mu}{\partial T} \frac{\partial T}{\partial z}\right) .
\end{aligned}
$$

\section{E.2 Mathematical Modeling for Energy}

In a similar manner, the unsteady state differential energy balance equation is as follows

$$
\begin{aligned}
\frac{\mathrm{d}\left(\triangle V \phi \rho C_{\mathrm{p}} T\right)}{\mathrm{d} t}= & \left(\triangle A v \rho C_{\mathrm{p}} T\right)_{z+\triangle z}-\left(\triangle A v \rho C_{\mathrm{p}} T\right)_{z}-\left(q_{r} \triangle S\right)_{r+\triangle r} \\
& +\left(q_{r} \triangle S\right)_{r}-\left(q_{z} \triangle A\right)_{z+\triangle z}+\left(q_{z} \triangle A\right)_{z}
\end{aligned}
$$


where, $C_{\mathrm{p}}$ is the specific heat of oil. In addition, $q_{r}$ and $q_{z}$ are thermal fluxes of air along radial and axial directions respectively. They are governed by the following constitutive relations

$$
q_{r}=-k \phi \frac{\partial T}{\partial r}
$$

and,

$$
q_{z}=-k \phi \frac{\partial T}{\partial z}
$$

where $k$ is the thermal conductivity. Moreover, we define $\gamma$ as the thermal diffusivity which is given by

$$
\gamma=\frac{k}{\rho C_{\mathrm{p}}}
$$

Like the previous case, by taking the limits of both $\Delta r$ and $\triangle z$ to zero ([56]), previous equations are framed as the following

$$
\frac{\partial T}{\partial t}=\frac{1}{\phi} \frac{\partial(v T)}{\partial z}+\frac{1}{r} \frac{\partial}{\partial r}\left(k r \frac{\partial T}{\partial r}\right)+\frac{\partial}{\partial z}\left(k \frac{\partial T}{\partial z}\right) .
$$

Thus,

$$
\frac{\partial T}{\partial t}=\frac{\alpha}{\phi \mu} \frac{\partial T}{\partial z}+\frac{\alpha T}{\phi} \frac{\partial}{\partial z}\left(\frac{1}{\mu}\right)+\frac{k}{r \rho C_{\mathrm{p}}} \frac{\partial T}{\partial r} \frac{\partial r}{\partial r}+\frac{k r}{r \rho C_{\mathrm{p}}} \frac{\partial}{\partial r}\left(\frac{\partial T}{\partial r}\right)+\frac{k}{\rho C_{\mathrm{p}}} \frac{\partial^{2} T}{\partial z^{2}} .
$$

We perform the derivative by considering dependency of viscosity on both temperature and concentration at a given pressure. Thus by applying chain rule of derivatives, we obtain

$$
\frac{\partial T}{\partial t}=\frac{\alpha}{\phi \mu} \frac{\partial T}{\partial z}-\frac{\alpha T}{\phi \mu^{2}}\left(\frac{\partial \mu}{\partial w} \frac{\partial w}{\partial z}+\frac{\partial \mu}{\partial T} \frac{\partial T}{\partial z}\right)+\frac{\gamma}{r} \frac{\partial T}{\partial r}+\gamma \frac{\partial^{2} T}{\partial r^{2}}+\gamma \frac{\partial^{2} T}{\partial z^{2}}
$$

which upon further rearrangement yields following energy balance model:

$$
\frac{\partial T}{\partial t}=\frac{\gamma}{r} \frac{\partial T}{\partial r}+\gamma \frac{\partial^{2} T}{\partial r^{2}}+\gamma \frac{\partial^{2} T}{\partial z^{2}}+\frac{\alpha}{\phi \mu} \frac{\partial T}{\partial z}-\frac{\alpha T}{\phi \mu^{2}}\left(\frac{\partial \mu}{\partial w} \frac{\partial w}{\partial z}+\frac{\partial \mu}{\partial T} \frac{\partial T}{\partial z}\right) .
$$




\section{E.3 Mathematical Modeling for Momentum}

Oil production necessarily reduces height $z(t, r)$ of bitumen in the packed cylindrical column. Starting from the momentum balance, the change in the height with time at any radial location is given by the negative of Darcy velocity $([56])$ at the bottom of the physical model as

$$
\frac{\partial(\triangle A z \phi \rho)}{\partial t}=-\rho v_{0}(r) \times 2 \pi r \triangle r
$$

Thus,

$$
\frac{\partial z}{\partial t}=-\frac{v_{0}(r)}{\phi}
$$

where $v_{0}(r)$ is the Darcy velocity at $z=0$. It is integrated over the differential element at $z=0$ and is represented by

$$
v_{0}=\frac{K_{\mathrm{r}} K \rho g}{\bar{\mu}}=\frac{\alpha}{\bar{\mu}} .
$$

Equations E.7, E.15 and E.17 represents mathematical models describing mass fraction, temperature and axial height (moving boundary) for the cylindrical physical geometry that is subjected under nitrogen injection enhanced oil recovery process. These equations have both time and spatial derivatives and therefore the solution depends on relevant initial conditions and boundary conditions. 


\section{Appendix F}

\section{Model Simulation Results}

As mentioned in Chapter 4, Section 4.2.1, we have three simultaneous, heterogeneous, second order, distributed parameter partial differential equations. Here, we discuss the model simulation results for three state variables for $163.85 \mathrm{~min}$ at different grid points along the cylindrical geometry at the operating pressure of 74.5 psi and $90^{\circ} \mathrm{C}$.

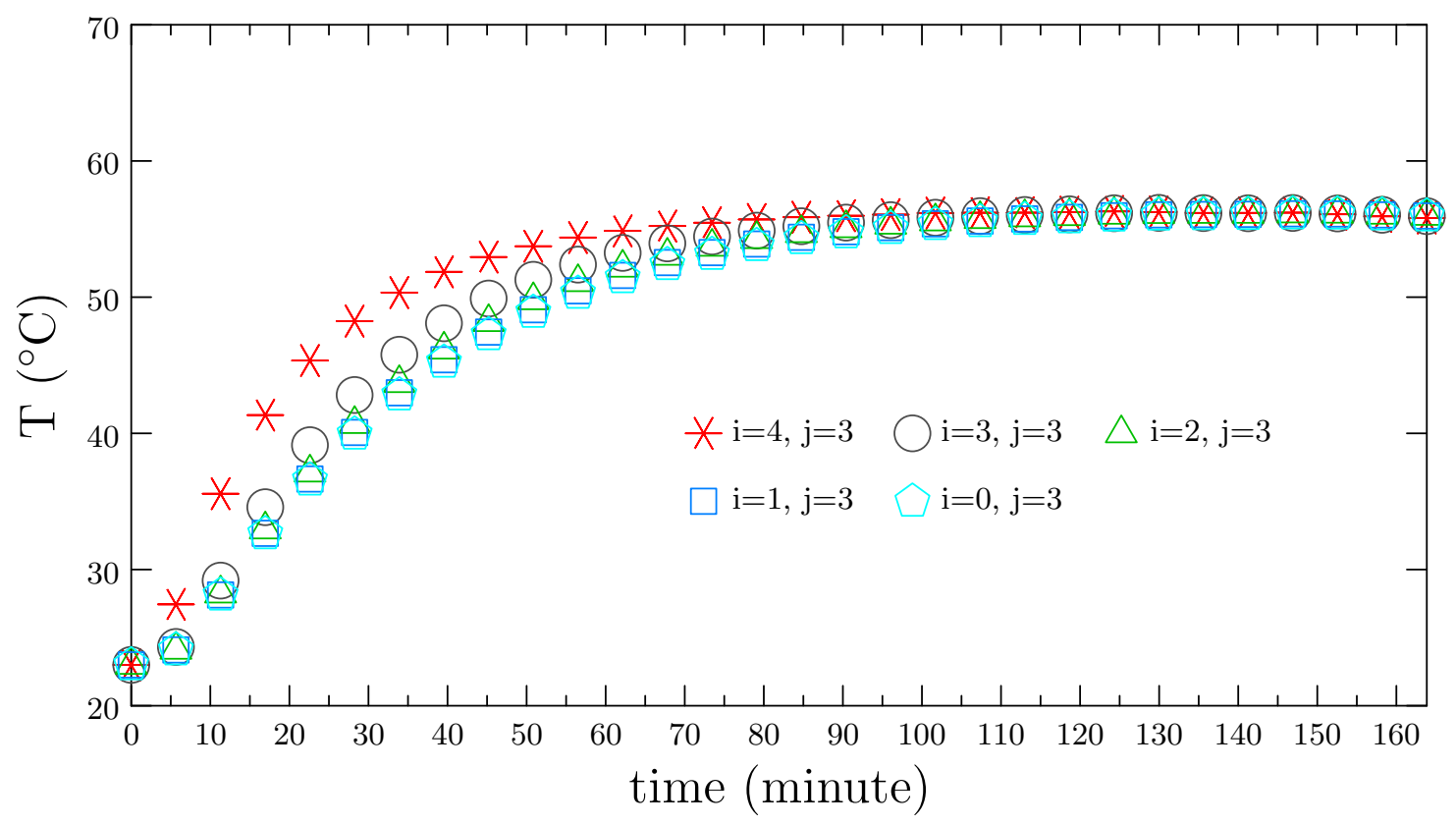

Figure F.1: Temperature versus time.

We observe that at all grid points, temperature attains steady state after 80 min. Initially, the curved surface (grid point $i=4$ and $j=3$ ) gets more heated up than the inner core as shown 
in Figure F.1. But neither mass fraction (Figure F.2) nor axial distance (Figure F.3) attains steady state during this observation period.

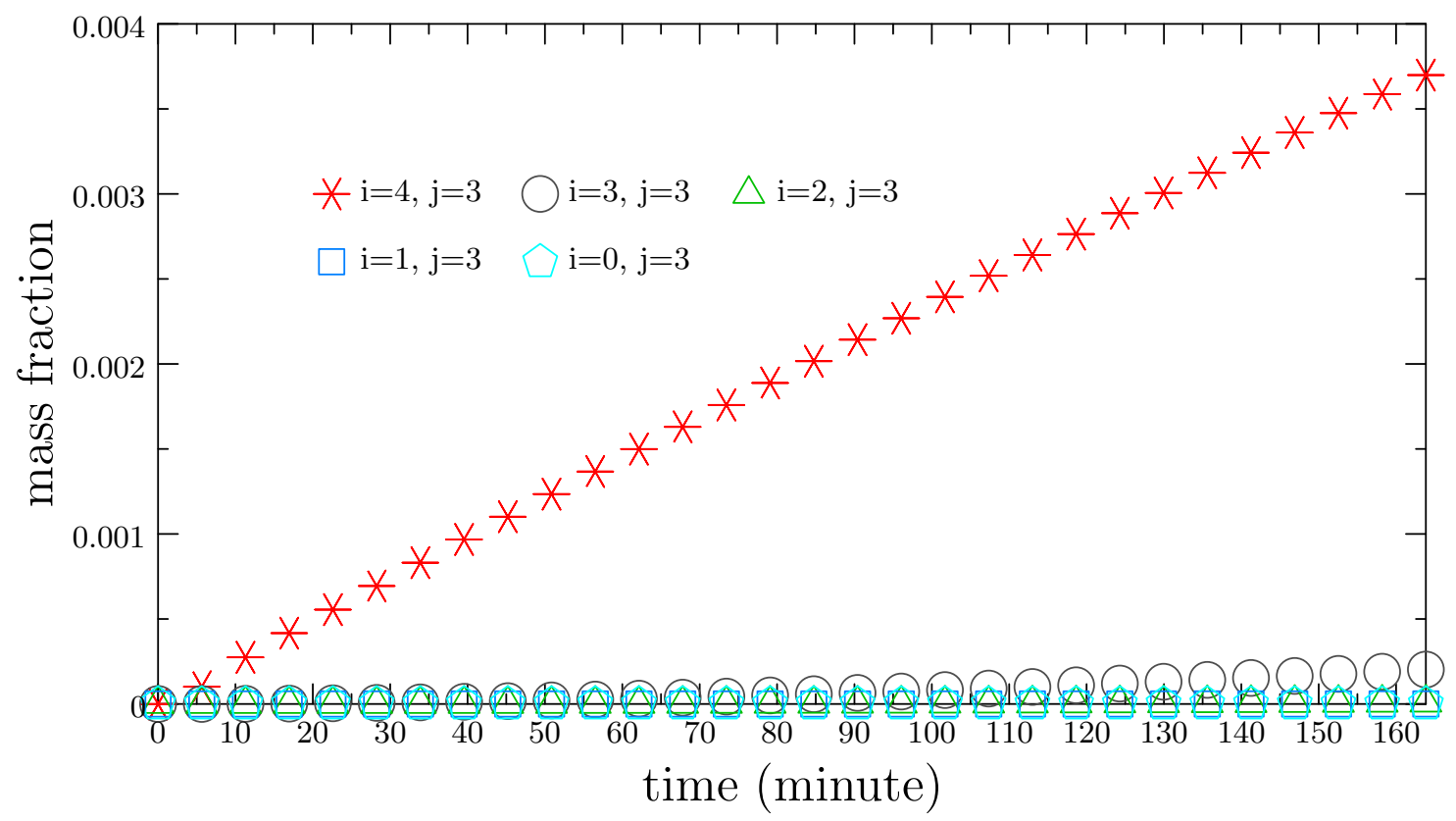

Figure F.2: Mass Fraction versus time.

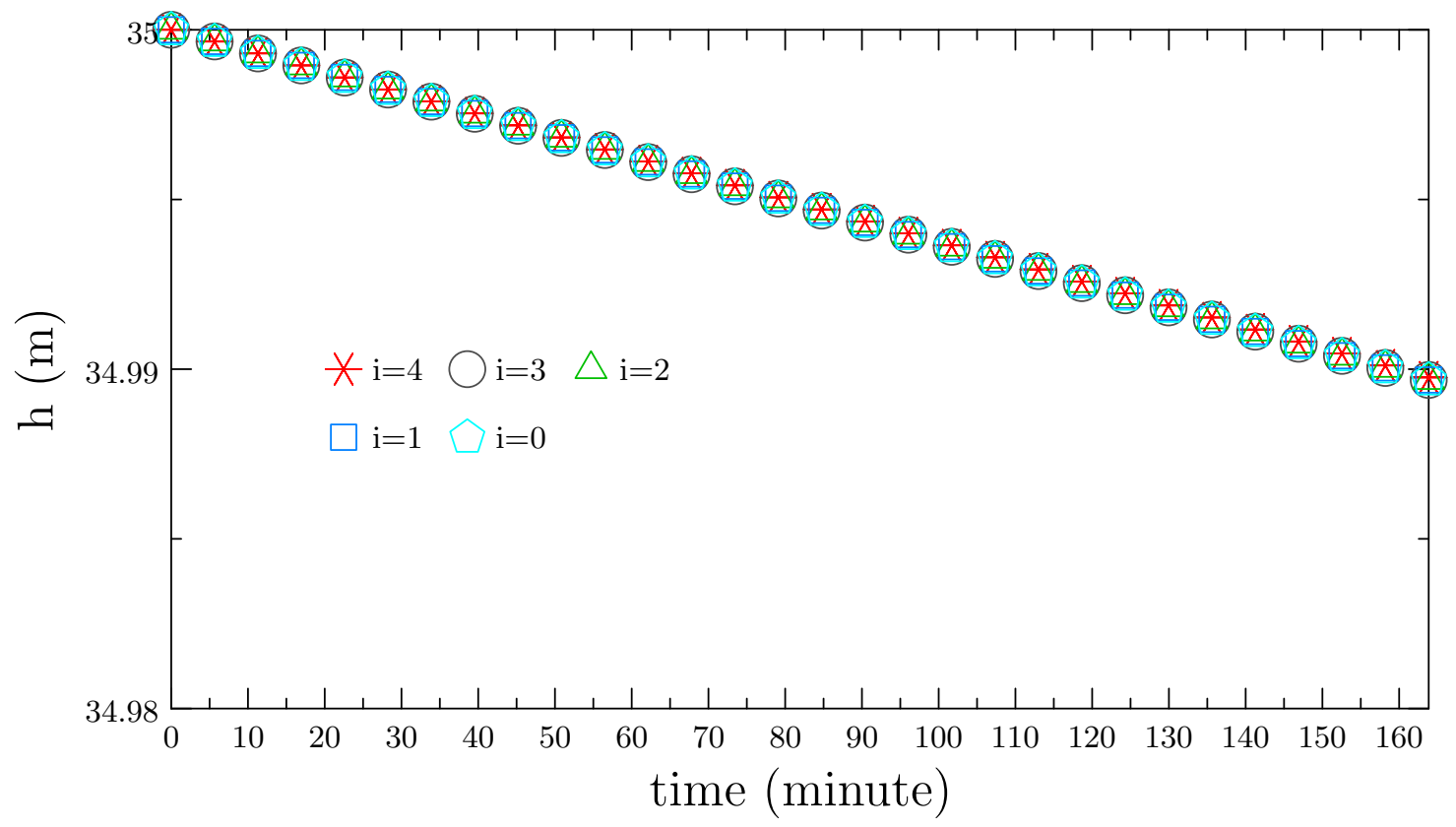

Figure F.3: Axial height versus time. 
We can see that concentration (mass fraction) gets steadily increased with time and dissolution of nitrogen is considerably high in the periphery (curved surface at $r=R$ ) as compared to the inner core (as seen in Figure F.2). The main reason for this phenomenon is: the curved surface is completely exposed to the flow of nitrogen; that's why dissolution resistance is very low at the boundaries and nitrogen dissolution is very rapid. Figure F.3 shows that height steadily decreases with time and aids in recovery of heavy oil.

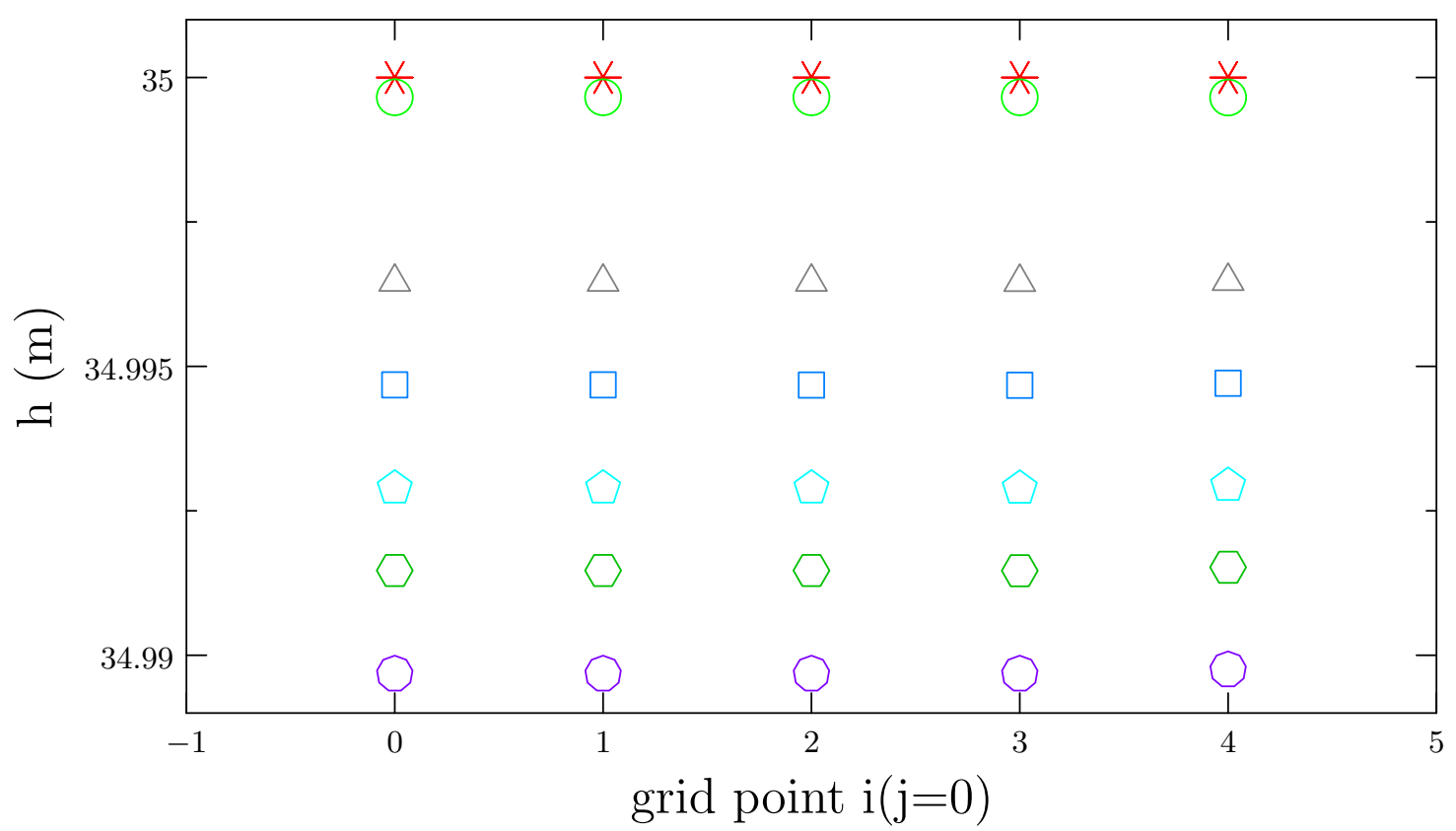

\begin{tabular}{|c|c|c|}
\hline$\forall$ time $=0$ minute & $\bigcirc$ time $=5.7$ minute & $\triangle$ time $=56.9$ minute \\
\hline$\square$ time $=85.3$ minute & $\square$ time $=113.8$ minute & $\square$ time $=136.6$ minute \\
\hline$\bigcirc$ time $=163.9$ minute & & \\
\hline
\end{tabular}

Figure F.4: Axial height versus time.

Moreover, Figure F.4 shows the variation of axial height at different grid locations along the radial $(i)$ direction at different time instants. 


\section{Appendix G}

\section{Derivation of Necessary Conditions}

As mentioned in Chapter 4, Section 4.2.3, for the necessary condition for the maximum of $J$, its variation $\delta J$ must be zero, that is,

$$
\begin{aligned}
\delta J=\delta I & +\int_{0}^{t_{\mathrm{f}}} \int_{0}^{R} \int_{0}^{Z_{0}}\left(\delta \lambda_{1} G_{1}+\lambda_{1} \delta G_{1}\right) \mathrm{d} z \mathrm{~d} r \mathrm{~d} t+\int_{0}^{t_{\mathrm{f}}} \int_{0}^{R} \int_{0}^{Z_{0}}\left(\delta \lambda_{2} G_{2}+\lambda_{2} \delta G_{2}\right) \mathrm{d} z \mathrm{~d} r \mathrm{~d} t \\
& +\int_{0}^{t_{\mathrm{f}}} \int_{0}^{R} \int_{0}^{Z_{0}} \frac{\left(\delta \lambda_{3} G_{3}+\lambda_{3} \delta G_{3}\right)}{Z_{0}} \mathrm{~d} z \mathrm{~d} r \mathrm{~d} t=0 .
\end{aligned}
$$

We expand $\delta G_{1}, \delta G_{2}$ and $\delta G_{3}$ in the previous equation by considering the following expressions (explicit variables):

$$
\begin{aligned}
& w_{r}=\frac{\partial w}{\partial r}, w_{z}=\frac{\partial w}{\partial z}, w_{r r}=\frac{\partial^{2} w}{\partial r^{2}} \text { and } w_{z z}=\frac{\partial^{2} w}{\partial z^{2}} ; \text { and } \\
& T_{r}=\frac{\partial T}{\partial r}, T_{z}=\frac{\partial T}{\partial z}, T_{r r}=\frac{\partial^{2} T}{\partial r^{2}} \text { and } T_{z z}=\frac{\partial^{2} T}{\partial z^{2}} .
\end{aligned}
$$

Moreover, while expanding, we have taken into consideration all explicitly available variables $(w, T, z)$ and their derivatives. Thus, we obtain 


$$
\begin{aligned}
\delta J= & \delta I-\int_{0}^{t_{\mathrm{f}}} \int_{0}^{R} \int_{0}^{Z_{0}}\left(\lambda_{1} \frac{\partial(\delta T)}{\partial t}+\lambda_{2} \frac{\partial(\delta w)}{\partial t}+\frac{\lambda_{3}}{Z_{0}} \frac{\partial(\delta z)}{\partial t}\right) \mathrm{d} z \mathrm{~d} r \mathrm{~d} t \\
& +\int_{0}^{t_{\mathrm{f}}} \int_{0}^{R} \int_{0}^{Z_{0}}\left(\lambda_{1} \frac{\partial f_{1}}{\partial w} \delta w+\lambda_{1} \frac{\partial f_{1}}{\partial T} \delta T+\lambda_{1} \frac{\partial f_{1}}{\partial w_{r}} \delta w_{r}+\lambda_{1} \frac{\partial f_{1}}{\partial w_{z}} \delta w_{z}+\lambda_{1} \frac{\partial f_{1}}{\partial T_{r}} \delta T_{r}\right. \\
& +\lambda_{1} \frac{\partial f_{1}}{\partial T_{z}} \delta T_{z}+\lambda_{1} \frac{\partial f_{1}}{\partial w_{r r}} \delta w_{r r}+\lambda_{1} \frac{\partial f_{1}}{\partial w_{z z}} \delta w_{z z}+\lambda_{1} \frac{\partial f_{1}}{\partial T_{r r}} \delta T_{r r} \\
& \left.+\lambda_{1} \frac{\partial f_{1}}{\partial T_{z z}} \delta T_{z z}\right) \mathrm{d} z \mathrm{~d} r \mathrm{~d} t+\int_{0}^{t_{\mathrm{f}}} \int_{0}^{R}\left(\lambda_{0} \frac{\partial f_{2}}{\partial w} \delta w+\lambda_{2} \frac{\partial f_{2}}{\partial T} \delta T+\lambda_{2} \frac{\partial f_{2}}{\partial w_{r}} \delta w_{r}\right. \\
& +\lambda_{2} \frac{\partial f_{2}}{\partial w_{z}} \delta w_{z}+\lambda_{2} \frac{\partial f_{2}}{\partial T_{r}} \delta T_{r}+\lambda_{2} \frac{\partial f_{2}}{\partial T_{z}} \delta T_{z}+\lambda_{2} \frac{\partial f_{2}}{\partial w_{r r}} \delta w_{r r}+\lambda_{2} \frac{\partial f_{2}}{\partial w_{z z}} \delta w_{z z} \\
+ & \left.\lambda_{2} \frac{\partial f_{2}}{\partial T_{r r}} \delta T_{r r}+\lambda_{2} \frac{\partial f_{2}}{\partial T_{z z}} \delta T_{z z}\right) \mathrm{d} z \mathrm{~d} r \mathrm{~d} t \\
& +\int_{0}^{t_{\mathrm{f}}} \int_{0}^{R} \int_{0}^{R}\left(G_{1} \delta \lambda_{1}+G_{2} \delta \lambda_{2}+\frac{G_{3}}{Z_{0}} \delta \lambda_{3}\right) \mathrm{d} z \mathrm{~d} r \mathrm{~d} t=0 . \\
+ & \int_{0}^{Z_{0}} \int_{0}^{z_{0}}\left(\frac{\lambda_{3}}{Z_{0}} \frac{\partial f_{3}}{\partial \bar{w}} \delta \bar{w}+\frac{\lambda_{3}}{Z_{0}} \frac{\partial f_{3}}{\partial \bar{T}} \delta \bar{T}\right) \mathrm{d} z \mathrm{~d} r \mathrm{~d} t
\end{aligned}
$$

Further simplification of the above equation will provide a set of equations necessary for the optimum. Applying integration by parts to the second integral with respect to $t$ of the previous equation, we obtain

$$
\int_{0}^{t_{\mathrm{f}}} \int_{0}^{R} \int_{0}^{Z_{0}} \lambda_{1} \frac{\partial(\delta T)}{\partial t} \mathrm{~d} z \mathrm{~d} r \mathrm{~d} t=\int_{0}^{R} \int_{0}^{Z_{0}}\left[\lambda_{1} \delta T\right]_{0}^{t_{\mathrm{f}}} \mathrm{d} z \mathrm{~d} r-\int_{0}^{t_{\mathrm{f}}} \int_{0}^{R} \int_{0}^{Z_{0}} \frac{\partial \lambda_{1}}{\partial t} \delta T \mathrm{~d} z \mathrm{~d} r \mathrm{~d} t .
$$

As initial temperature is fixed, $\delta T=0$ for all $r$ and $z$. Now if we specify [52, 53, 54]

$$
\lambda_{1}\left(t_{\mathrm{f}}, r, z\right)=0, \quad, \quad 0 \leq r \leq R \text { and } 0 \leq z \leq Z_{0}
$$


then the above integral simplifies to

$$
\int_{0}^{t_{\mathrm{f}}} \int_{0}^{R} \int_{0}^{Z_{0}} \lambda_{1} \frac{\partial(\delta T)}{\partial t} \mathrm{~d} z \mathrm{~d} r \mathrm{~d} t=-\int_{0}^{t_{\mathrm{f}}} \int_{0}^{R} \int_{0}^{Z_{0}} \frac{\partial \lambda_{1}}{\partial t} \delta T \mathrm{~d} z \mathrm{~d} r \mathrm{~d} t .
$$

By applying integration by parts to the third integral with respect to $t$ of equation G.4. we obtain

$$
\int_{0}^{t_{\mathrm{f}}} \int_{0}^{R} \int_{0}^{Z_{0}} \lambda_{2} \frac{\partial(\delta w)}{\partial t} \mathrm{~d} z \mathrm{~d} r \mathrm{~d} t=\int_{0}^{R} \int_{0}^{Z_{0}}\left[\lambda_{2} \delta w\right]_{0}^{t_{\mathrm{f}}} \mathrm{d} z \mathrm{~d} r-\int_{0}^{t_{\mathrm{f}}} \int_{0}^{R} \int_{0}^{Z_{0}} \frac{\partial \lambda_{2}}{\partial t} \delta w \mathrm{~d} z \mathrm{~d} r \mathrm{~d} t .
$$

As initial nitrogen mass fraction is fixed, $\delta w=0$ for all $r$ and $z$. Now if we specify

$$
\lambda_{2}\left(t_{\mathrm{f}}, r, z\right)=0, \quad 0 \leq r \leq R \text { and } 0 \leq z \leq Z_{0}
$$

then the above integral simplifies to

$$
\int_{0}^{t_{\mathrm{f}}} \int_{0}^{R} \int_{0}^{Z_{0}} \lambda_{2} \frac{\partial(\delta w)}{\partial t} \mathrm{~d} z \mathrm{~d} r \mathrm{~d} t=-\int_{0}^{t_{\mathrm{f}}} \int_{0}^{R} \int_{0}^{Z_{0}} \frac{\partial \lambda_{2}}{\partial t} \delta w \mathrm{~d} z \mathrm{~d} r \mathrm{~d} t
$$

Similarly, applying integration by parts to the fourth integral with respect to $t$ of equation G.4, we obtain

$$
\int_{0}^{t_{\mathrm{f}}} \int_{0}^{R} \int_{0}^{Z_{0}} \frac{\lambda_{3}}{Z_{0}} \frac{\partial(\delta z)}{\partial t} \mathrm{~d} z \mathrm{~d} r \mathrm{~d} t=\int_{0}^{R} \int_{0}^{Z_{0}}\left[\frac{\lambda_{3}}{Z_{0}} \delta z\right]_{0}^{t_{\mathrm{f}}} \mathrm{d} z \mathrm{~d} r-\int_{0}^{t_{\mathrm{f}}} \int_{0}^{R} \int_{0}^{Z_{0}} \frac{1}{Z_{0}} \frac{\partial \lambda_{3}}{\partial t} \delta z \mathrm{~d} z \mathrm{~d} r \mathrm{~d} t .
$$

As initial height is fixed, $\delta z=0$ for all $r$ and $z$. Now if we specify

$$
\lambda_{3}\left(t_{\mathrm{f}}, r\right)=0, \quad 0 \leq r \leq R
$$

then the above integral simplifies to

$$
\int_{0}^{t_{\mathrm{f}}} \int_{0}^{R} \int_{0}^{Z_{0}} \frac{\lambda_{3}}{Z_{0}} \frac{\partial(\delta z)}{\partial t} \mathrm{~d} z \mathrm{~d} r \mathrm{~d} t=-\int_{0}^{t_{\mathrm{f}}} \int_{0}^{R} \int_{0}^{Z_{0}} \frac{1}{Z_{0}} \frac{\partial \lambda_{3}}{\partial t} \delta z \mathrm{~d} z \mathrm{~d} r \mathrm{~d} t=-\int_{0}^{t_{\mathrm{f}}} \int_{0}^{R} \frac{1}{Z_{0}} \frac{\partial \lambda_{3}}{\partial t} \delta z \mathrm{~d} r \mathrm{~d} t .
$$


Integration by parts on the seventh integral with respect to $r$ of equation G.4. we obtain

$$
\begin{aligned}
\int_{0}^{t_{\mathrm{f}}} \int_{0}^{R} \int_{0}^{Z_{0}} \lambda_{1} \frac{\partial f_{1}}{\partial w_{r}} \delta w_{r} \mathrm{~d} z \mathrm{~d} r \mathrm{~d} t= & \int_{0}^{t_{\mathrm{f}}} \int_{0}^{Z_{0}}\left[\lambda_{1} \frac{\partial f_{1}}{\partial w_{r}} \delta w\right]_{0}^{R} \mathrm{~d} z \mathrm{~d} t \\
& -\int_{0}^{t_{\mathrm{f}}} \int_{0}^{R} \int_{0}^{Z_{0}} \frac{\partial}{\partial r}\left(\lambda_{1} \frac{\partial f_{1}}{\partial w_{r}}\right) \delta w \mathrm{~d} z \mathrm{~d} r \mathrm{~d} t .
\end{aligned}
$$

Considering that $\delta w$ is arbitrary at $r=0$ and the fact that all additive terms of $\delta J$ must be individually zero [52, 53, 54], it follows that

$$
\lambda_{1}(t, 0, z)=0, \quad, \quad 0 \leq t \leq t_{\mathrm{f}} \text { and } 0 \leq z \leq Z_{0}
$$

which yields

$$
\begin{aligned}
\int_{0}^{t_{\mathrm{f}}} \int_{0}^{R} \int_{0}^{Z_{0}} \lambda_{1} \frac{\partial f_{1}}{\partial w_{r}} \delta w_{r} \mathrm{~d} z \mathrm{~d} r \mathrm{~d} t= & \int_{0}^{t_{\mathrm{f}}} \int_{0}^{Z_{0}}\left[\lambda_{1} \frac{\partial f_{1}}{\partial w_{r}} \delta w\right]_{R} \mathrm{~d} z \mathrm{~d} t \\
& -\int_{0}^{t_{\mathrm{f}}} \int_{0}^{R} \int_{0}^{Z_{0}} \frac{\partial}{\partial r}\left(\lambda_{1} \frac{\partial f_{1}}{\partial w_{r}}\right) \delta w \mathrm{~d} z \mathrm{~d} r \mathrm{~d} t .
\end{aligned}
$$

Similarly, integration by parts on the eighth integral with respect to $z$ of equation G.4. we obtain

$$
\begin{aligned}
\int_{0}^{t_{\mathrm{f}}} \int_{0}^{R} \int_{0}^{Z_{0}} \lambda_{1} \frac{\partial f_{1}}{\partial w_{z}} \delta w_{z} \mathrm{~d} z \mathrm{~d} r \mathrm{~d} t= & \int_{0}^{t_{\mathrm{f}}} \int_{0}^{R}\left[\lambda_{1} \frac{\partial f_{1}}{\partial w_{z}} \delta w\right]_{0}^{Z_{0}} \mathrm{~d} r \mathrm{~d} t \\
& -\int_{0}^{t_{\mathrm{f}}} \int_{0}^{R} \int_{0}^{Z_{0}} \frac{\partial}{\partial z}\left(\lambda_{1} \frac{\partial f_{1}}{\partial w_{z}}\right) \delta w \mathrm{~d} z \mathrm{~d} r \mathrm{~d} t .
\end{aligned}
$$

Integration by parts on the ninth integral with respect to $r$ of equation G.4, we obtain

$$
\begin{aligned}
\int_{0}^{t_{\mathrm{f}}} \int_{0}^{R} \int_{0}^{Z_{0}} \lambda_{1} \frac{\partial f_{1}}{\partial T_{r}} \delta T_{r} \mathrm{~d} z \mathrm{~d} r \mathrm{~d} t= & \int_{0}^{t_{\mathrm{f}}} \int_{0}^{Z_{0}}\left[\lambda_{1} \frac{\partial f_{1}}{\partial T_{r}} \delta T\right]_{0}^{R} \mathrm{~d} z \mathrm{~d} t \\
& -\int_{0}^{t_{\mathrm{f}}} \int_{0}^{R} \int_{0}^{Z_{0}} \frac{\partial}{\partial r}\left(\lambda_{1} \frac{\partial f_{1}}{\partial T_{r}}\right) \delta T \mathrm{~d} z \mathrm{~d} r \mathrm{~d} t .
\end{aligned}
$$


Considering that $\delta T$ is arbitrary at $r=0$ and the fact that all additive terms of $\delta J$ must be individually zero, it follows that

$$
\lambda_{1}(t, 0, z)=0, \quad, \quad 0 \leq t \leq t_{\mathrm{f}} \text { and } 0 \leq z \leq Z_{0}
$$

which yields

$$
\begin{aligned}
\int_{0}^{t_{\mathrm{f}}} \int_{0}^{R} \int_{0}^{Z_{0}} \lambda_{1} \frac{\partial f_{1}}{\partial T_{r}} \delta T_{r} \mathrm{~d} z \mathrm{~d} r \mathrm{~d} t= & \int_{0}^{t_{\mathrm{f}}} \int_{0}^{Z_{0}}\left[\lambda_{1} \frac{\partial f_{1}}{\partial T_{r}} \delta T\right]_{R} \mathrm{~d} z \mathrm{~d} t \\
& -\int_{0}^{t_{\mathrm{f}}} \int_{0}^{R} \int_{0}^{Z_{0}} \frac{\partial}{\partial r}\left(\lambda_{1} \frac{\partial f_{1}}{\partial T_{r}}\right) \delta T \mathrm{~d} z \mathrm{~d} r \mathrm{~d} t .
\end{aligned}
$$

Integration by parts on the tenth integral with respect to $z$ of equation G.4. we obtain

$$
\begin{aligned}
\int_{0}^{t_{\mathrm{f}}} \int_{0}^{R} \int_{0}^{Z_{0}} \lambda_{1} \frac{\partial f_{1}}{\partial T_{z}} \delta T_{z} \mathrm{~d} z \mathrm{~d} r \mathrm{~d} t= & \int_{0}^{t_{\mathrm{f}}} \int_{0}^{R}\left[\lambda_{1} \frac{\partial f_{1}}{\partial T_{z}} \delta T\right]_{0}^{Z_{0}} \mathrm{~d} r \mathrm{~d} t \\
& -\int_{0}^{t_{\mathrm{f}}} \int_{0}^{R} \int_{0}^{Z_{0}} \frac{\partial}{\partial z}\left(\lambda_{1} \frac{\partial f_{1}}{\partial T_{z}}\right) \delta T \mathrm{~d} z \mathrm{~d} r \mathrm{~d} t .
\end{aligned}
$$

Integration by parts on the eleventh integral with respect to $r$ of equation G.4 we obtain

$$
\begin{aligned}
\int_{0}^{t_{\mathrm{f}}} \int_{0}^{R} \int_{0}^{Z_{0}} \lambda_{1} \frac{\partial f_{1}}{\partial w_{r r}} \delta w_{r r} \mathrm{~d} z \mathrm{~d} r \mathrm{~d} t= & \int_{0}^{t_{\mathrm{f}}} \int_{0}^{Z_{0}}\left[\lambda_{1} \frac{\partial f_{1}}{\partial w_{r r}} \delta w_{r}\right]_{0}^{R} \mathrm{~d} z \mathrm{~d} t \\
& -\int_{0}^{t_{\mathrm{f}}} \int_{0}^{R} \int_{0}^{Z_{0}} \frac{\partial}{\partial r}\left(\lambda_{1} \frac{\partial f_{1}}{\partial w_{r r}}\right) \delta w_{r} \mathrm{~d} z \mathrm{~d} r \mathrm{~d} t .
\end{aligned}
$$

Integration by parts of the second integral of the previous equation with respect to $r$ results 
in

$$
\begin{aligned}
\int_{0}^{t_{\mathrm{f}}} \int_{0}^{R} \int_{0}^{Z_{0}} \lambda_{1} \frac{\partial f_{1}}{\partial w_{r r}} \delta w_{r r} \mathrm{~d} z \mathrm{~d} r \mathrm{~d} t= & \int_{0}^{t_{\mathrm{f}}} \int_{0}^{Z_{0}}\left[\lambda_{1} \frac{\partial f_{1}}{\partial w_{r r}} \delta w_{r}\right]_{0}^{R} \mathrm{~d} z \mathrm{~d} t \\
& -\int_{0}^{t_{\mathrm{f}}} \int_{0}^{Z_{0}}\left[\frac{\partial}{\partial r}\left(\lambda_{1} \frac{\partial f_{1}}{\partial w_{r r}}\right) \delta w\right]_{0}^{R} \mathrm{~d} z \mathrm{~d} t \\
& +\int_{0}^{t_{\mathrm{f}}} \int_{0}^{R} \int_{0}^{Z_{0}} \frac{\partial^{2}}{\partial r^{2}}\left(\lambda_{1} \frac{\partial f_{1}}{\partial w_{r r}}\right) \delta w \mathrm{~d} z \mathrm{~d} r \mathrm{~d} t .
\end{aligned}
$$

Considering that $\delta w$ is arbitrary at $r=R$ and the fact that all additive terms of $\delta J$ must be individually zero, it follows that

$$
\lambda_{1}(t, R, z)=0, \quad, \quad 0 \leq t \leq t_{\mathrm{f}} \text { and } 0 \leq z \leq Z_{0}
$$

Keeping in mind that $w_{r}$ is zero at $r=0$ because of symmetry around the $z$-axis and making use of these two conditions, previous expression gets simplified to

$$
\begin{aligned}
\int_{0}^{t_{\mathrm{f}}} \int_{0}^{R} \int_{0}^{Z_{0}} \lambda_{1} \frac{\partial f_{1}}{\partial w_{r r}} \delta w_{r r} \mathrm{~d} z \mathrm{~d} r \mathrm{~d} t= & -\int_{0}^{t_{\mathrm{f}}} \int_{0}^{Z_{0}}\left[\frac{\partial}{\partial r}\left(\lambda_{1} \frac{\partial f_{1}}{\partial w_{r r}}\right) \delta w\right]_{R} \mathrm{~d} z \mathrm{~d} t \\
& +\int_{0}^{t_{\mathrm{f}}} \int_{0}^{R} \int_{0}^{Z_{0}} \frac{\partial^{2}}{\partial r^{2}}\left(\lambda_{1} \frac{\partial f_{1}}{\partial w_{r r}}\right) \delta w \mathrm{~d} z \mathrm{~d} r \mathrm{~d} t .
\end{aligned}
$$

Integration by parts on the thirteenth integral with respect to $r$ of equation G.4. we obtain

$$
\begin{aligned}
\int_{0}^{t_{\mathrm{f}}} \int_{0}^{R} \int_{0}^{Z_{0}} \lambda_{1} \frac{\partial f_{1}}{\partial T_{r r}} \delta T_{r r} \mathrm{~d} z \mathrm{~d} r \mathrm{~d} t= & \int_{0}^{t_{\mathrm{f}}} \int_{0}^{Z_{0}}\left[\lambda_{1} \frac{\partial f_{1}}{\partial T_{r r}} \delta T_{r}\right]_{0}^{R} \mathrm{~d} z \mathrm{~d} t \\
& -\int_{0}^{t_{\mathrm{f}}} \int_{0}^{R} \int_{0}^{Z_{0}} \frac{\partial}{\partial r}\left(\lambda_{1} \frac{\partial f_{1}}{\partial T_{r r}}\right) \delta T_{r} \mathrm{~d} z \mathrm{~d} r \mathrm{~d} t .
\end{aligned}
$$


Integration by parts of the second integral of the previous equation with respect to $\mathrm{r}$ results in

$$
\begin{aligned}
\int_{0}^{t_{\mathrm{f}}} \int_{0}^{R} \int_{0}^{Z_{0}} \lambda_{1} \frac{\partial f_{1}}{\partial T_{r r}} \delta T_{r r} \mathrm{~d} z \mathrm{~d} r \mathrm{~d} t= & \int_{0}^{t_{\mathrm{f}}} \int_{0}^{Z_{0}}\left[\lambda_{1} \frac{\partial f_{1}}{\partial T_{r r}} \delta T_{r}\right]_{0}^{R} \mathrm{~d} z \mathrm{~d} t \\
& -\int_{0}^{t_{\mathrm{f}}} \int_{0}^{Z_{0}}\left[\frac{\partial}{\partial r}\left(\lambda_{1} \frac{\partial f_{1}}{\partial T_{r r}}\right) \delta T\right]_{0}^{R} \mathrm{~d} z \mathrm{~d} t \\
& +\int_{0}^{t_{\mathrm{f}}} \int_{0}^{R} \int_{0}^{Z_{0}} \frac{\partial^{2}}{\partial r^{2}}\left(\lambda_{1} \frac{\partial f_{1}}{\partial T_{r r}}\right) \delta T \mathrm{~d} z \mathrm{~d} r \mathrm{~d} t .
\end{aligned}
$$

Considering that $\delta T$ is arbitrary at $r=R$ and the fact that all additive terms of $\delta J$ must be individually zero, it follows that

$$
\lambda_{1}(t, R, z)=0, \quad, 0 \leq t \leq t_{\mathrm{f}} \text { and } 0 \leq z \leq Z_{0}
$$

Keeping in mind that $T_{r}$ is zero at $r=0$ because of symmetry around the z-axis and making use of these two conditions, previous expression gets simplified to

$$
\begin{aligned}
\int_{0}^{t_{\mathrm{f}}} \int_{0}^{R} \int_{0}^{Z_{0}} \lambda_{1} \frac{\partial f_{1}}{\partial T_{r r}} \delta T_{r r} \mathrm{~d} z \mathrm{~d} r \mathrm{~d} t= & -\int_{0}^{t_{\mathrm{f}}} \int_{0}^{Z_{0}}\left[\frac{\partial}{\partial r}\left(\lambda_{1} \frac{\partial f_{1}}{\partial T_{r r}}\right) \delta T\right]_{R} \mathrm{~d} z \mathrm{~d} t \\
& +\int_{0}^{t_{\mathrm{f}}} \int_{0}^{R} \int_{0}^{Z_{0}} \frac{\partial^{2}}{\partial r^{2}}\left(\lambda_{1} \frac{\partial f_{1}}{\partial T_{r r}}\right) \delta T \mathrm{~d} z \mathrm{~d} r \mathrm{~d} t .
\end{aligned}
$$

Integration by parts on the twelfth integral with respect to $z$ of equation G.4. we obtain

$$
\begin{aligned}
\int_{0}^{t_{\mathrm{f}}} \int_{0}^{R} \int_{0}^{Z_{0}} \lambda_{1} \frac{\partial f_{1}}{\partial w_{z z}} \delta w_{z z} \mathrm{~d} z \mathrm{~d} r \mathrm{~d} t= & \int_{0}^{t_{\mathrm{f}}} \int_{0}^{R}\left[\lambda_{1} \frac{\partial f_{1}}{\partial w_{z z}} \delta w_{z}\right]_{0}^{Z_{0}} \mathrm{~d} r \mathrm{~d} t \\
& -\int_{0}^{t_{\mathrm{f}}} \int_{0}^{R} \int_{0}^{Z_{0}} \frac{\partial}{\partial z}\left(\lambda_{1} \frac{\partial f_{1}}{\partial w_{z z}}\right) \delta w_{z} \mathrm{~d} z \mathrm{~d} r \mathrm{~d} t .
\end{aligned}
$$


Integration by parts of the second integral of the previous equation with respect to $z$ results in

$$
\begin{aligned}
\int_{0}^{t_{\mathrm{f}}} \int_{0}^{R} \int_{0}^{Z_{0}} \lambda_{1} \frac{\partial f_{1}}{\partial w_{z z}} \delta w_{z z} \mathrm{~d} z \mathrm{~d} r \mathrm{~d} t= & \int_{0}^{t_{\mathrm{f}}} \int_{0}^{R}\left[\lambda_{1} \frac{\partial f_{1}}{\partial w_{z z}} \delta w_{z}\right]_{0}^{Z_{0}} \mathrm{~d} r \mathrm{~d} t \\
& -\int_{0}^{t_{\mathrm{f}}} \int_{0}^{R}\left[\frac{\partial}{\partial z}\left(\lambda_{1} \frac{\partial f_{1}}{\partial w_{z z}}\right) \delta w\right]_{0}^{Z_{0}} \mathrm{~d} r \mathrm{~d} t \\
& +\int_{0}^{t_{\mathrm{f}}} \int_{0}^{R} \int_{0}^{Z_{0}} \frac{\partial^{2}}{\partial z^{2}}\left(\lambda_{1} \frac{\partial f_{1}}{\partial w_{z z}}\right) \delta w \mathrm{~d} z \mathrm{~d} r \mathrm{~d} t .
\end{aligned}
$$

Integration by parts on the fourteenth integral with respect to $z$ of equation G.4, we obtain

$$
\begin{aligned}
\int_{0}^{t_{\mathrm{f}}} \int_{0}^{R} \int_{0}^{Z_{0}} \lambda_{1} \frac{\partial f_{1}}{\partial T_{z z}} \delta T_{z z} \mathrm{~d} z \mathrm{~d} r \mathrm{~d} t= & \int_{0}^{t_{\mathrm{f}}} \int_{0}^{R}\left[\lambda_{1} \frac{\partial f_{1}}{\partial T_{z z}} \delta T_{z}\right]_{0}^{Z_{0}} \mathrm{~d} r \mathrm{~d} t \\
& -\int_{0}^{t_{\mathrm{f}}} \int_{0}^{R} \int_{0}^{Z_{0}} \frac{\partial}{\partial z}\left(\lambda_{1} \frac{\partial f_{1}}{\partial T_{z z}}\right) \delta T_{z} \mathrm{~d} z \mathrm{~d} r \mathrm{~d} t .
\end{aligned}
$$

Integration by parts of the second integral of the previous equation with respect to $z$ results in

$$
\begin{aligned}
\int_{0}^{t_{\mathrm{f}}} \int_{0}^{R} \int_{0}^{Z_{0}} \lambda_{1} \frac{\partial f_{1}}{\partial T_{z z}} \delta T_{z z} \mathrm{~d} z \mathrm{~d} r \mathrm{~d} t= & \int_{0}^{t_{\mathrm{f}}} \int_{0}^{R}\left[\lambda_{1} \frac{\partial f_{1}}{\partial T_{z z}} \delta T_{z}\right]_{0}^{Z_{0}} \mathrm{~d} r \mathrm{~d} t \\
& -\int_{0}^{t_{\mathrm{f}}} \int_{0}^{R}\left[\frac{\partial}{\partial z}\left(\lambda_{1} \frac{\partial f_{1}}{\partial T_{z z}}\right) \delta T\right]_{0}^{Z_{0}} \mathrm{~d} r \mathrm{~d} t \\
& +\int_{0}^{t_{\mathrm{f}}} \int_{0}^{R} \int_{0}^{Z_{0}} \frac{\partial^{2}}{\partial z^{2}}\left(\lambda_{1} \frac{\partial f_{1}}{\partial T_{z z}}\right) \delta T \mathrm{~d} z \mathrm{~d} r \mathrm{~d} t .
\end{aligned}
$$


Integration by parts on the seventeenth integral with respect to $r$ of equation G.4, we obtain

$$
\begin{aligned}
\int_{0}^{t_{\mathrm{f}}} \int_{0}^{R} \int_{0}^{Z_{0}} \lambda_{2} \frac{\partial f_{2}}{\partial w_{r}} \delta w_{r} \mathrm{~d} z \mathrm{~d} r \mathrm{~d} t= & \int_{0}^{t_{\mathrm{f}}} \int_{0}^{Z_{0}}\left[\lambda_{2} \frac{\partial f_{2}}{\partial w_{r}} \delta w\right]_{0}^{R} \mathrm{~d} z \mathrm{~d} t \\
& -\int_{0}^{t_{\mathrm{f}}} \int_{0}^{R} \int_{0}^{Z_{0}} \frac{\partial}{\partial r}\left(\lambda_{2} \frac{\partial f_{2}}{\partial w_{r}}\right) \delta w \mathrm{~d} z \mathrm{~d} r \mathrm{~d} t .
\end{aligned}
$$

Considering that $\delta w$ is arbitrary at $r=0$ and the fact that all additive terms of $\delta J$ must be individually zero [52, 53, 54], it follows that

$$
\lambda_{2}(t, 0, z)=0, \quad, \quad 0 \leq t \leq t_{\mathrm{f}} \text { and } 0 \leq z \leq Z_{0}
$$

which yields

$$
\begin{aligned}
\int_{0}^{t_{\mathrm{f}}} \int_{0}^{R} \int_{0}^{Z_{0}} \lambda_{2} \frac{\partial f_{2}}{\partial w_{r}} \delta w_{r} \mathrm{~d} z \mathrm{~d} r \mathrm{~d} t= & \int_{0}^{t_{\mathrm{f}}} \int_{0}^{Z_{0}}\left[\lambda_{2} \frac{\partial f_{2}}{\partial w_{r}} \delta w\right]_{R} \mathrm{~d} z \mathrm{~d} t \\
& -\int_{0}^{t_{\mathrm{f}}} \int_{0}^{R} \int_{0}^{Z_{0}} \frac{\partial}{\partial r}\left(\lambda_{2} \frac{\partial f_{2}}{\partial w_{r}}\right) \delta w \mathrm{~d} z \mathrm{~d} r \mathrm{~d} t .
\end{aligned}
$$

Similarly, integration by parts on the eighteenth integral with respect to $z$ of equation G.4, we obtain

$$
\begin{aligned}
\int_{0}^{t_{\mathrm{f}}} \int_{0}^{R} \int_{0}^{Z_{0}} \lambda_{2} \frac{\partial f_{2}}{\partial w_{z}} \delta w_{z} \mathrm{~d} z \mathrm{~d} r \mathrm{~d} t= & \int_{0}^{t_{\mathrm{f}}} \int_{0}^{R}\left[\lambda_{2} \frac{\partial f_{2}}{\partial w_{z}} \delta w\right]_{0}^{Z_{0}} \mathrm{~d} r \mathrm{~d} t \\
& -\int_{0}^{t_{\mathrm{f}}} \int_{0}^{R} \int_{0}^{Z_{0}} \frac{\partial}{\partial z}\left(\lambda_{2} \frac{\partial f_{2}}{\partial w_{z}}\right) \delta w \mathrm{~d} z \mathrm{~d} r \mathrm{~d} t
\end{aligned}
$$

Integration by parts on the nineteenth integral with respect to $r$ of equation G.4. we 
obtain

$$
\begin{aligned}
\int_{0}^{t_{\mathrm{f}}} \int_{0}^{R} \int_{0}^{Z_{0}} \lambda_{2} \frac{\partial f_{2}}{\partial T_{r}} \delta T_{r} \mathrm{~d} z \mathrm{~d} r \mathrm{~d} t= & \int_{0}^{t_{\mathrm{f}}} \int_{0}^{Z_{0}}\left[\lambda_{2} \frac{\partial f_{2}}{\partial T_{r}} \delta T\right]_{0}^{R} \mathrm{~d} z \mathrm{~d} t \\
& -\int_{0}^{t_{\mathrm{f}}} \int_{0}^{R} \int_{0}^{Z_{0}} \frac{\partial}{\partial r}\left(\lambda_{2} \frac{\partial f_{2}}{\partial T_{r}}\right) \delta T \mathrm{~d} z \mathrm{~d} r \mathrm{~d} t
\end{aligned}
$$

Considering that $\delta T$ is arbitrary at $r=0$ and the fact that all additive terms of $\delta J$ must be individually zero, it follows that

$$
\lambda_{2}(t, 0, z)=0, \quad, \quad 0 \leq t \leq t_{\mathrm{f}} \text { and } 0 \leq z \leq Z_{0}
$$

which yields

$$
\begin{aligned}
\int_{0}^{t_{\mathrm{f}}} \int_{0}^{R} \int_{0}^{Z_{0}} \lambda_{2} \frac{\partial f_{2}}{\partial T_{r}} \delta T_{r} \mathrm{~d} z \mathrm{~d} r \mathrm{~d} t= & \int_{0}^{t_{\mathrm{f}}} \int_{0}^{Z_{0}}\left[\lambda_{2} \frac{\partial f_{2}}{\partial T_{r}} \delta T\right]_{R} \mathrm{~d} z \mathrm{~d} t \\
& -\int_{0}^{t_{\mathrm{f}}} \int_{0}^{R} \int_{0}^{Z_{0}} \frac{\partial}{\partial r}\left(\lambda_{2} \frac{\partial f_{2}}{\partial T_{r}}\right) \delta T \mathrm{~d} z \mathrm{~d} r \mathrm{~d} t .
\end{aligned}
$$

Integration by parts on the twentieth integral with respect to $z$ of equation G.4, we obtain

$$
\begin{aligned}
\int_{0}^{t_{\mathrm{f}}} \int_{0}^{R} \int_{0}^{Z_{0}} \lambda_{2} \frac{\partial f_{2}}{\partial T_{z}} \delta T_{z} \mathrm{~d} z \mathrm{~d} r \mathrm{~d} t= & \int_{0}^{t_{\mathrm{f}}} \int_{0}^{R}\left[\lambda_{2} \frac{\partial f_{2}}{\partial T_{z}} \delta T\right]_{0}^{Z_{0}} \mathrm{~d} r \mathrm{~d} t \\
& -\int_{0}^{t_{\mathrm{f}}} \int_{0}^{R} \int_{0}^{Z_{0}} \frac{\partial}{\partial z}\left(\lambda_{2} \frac{\partial f_{2}}{\partial T_{z}}\right) \delta T \mathrm{~d} z \mathrm{~d} r \mathrm{~d} t .
\end{aligned}
$$

Integration by parts on the twenty first integral with respect to $r$ of equation G.4. we obtain

$$
\begin{aligned}
\int_{0}^{t_{\mathrm{f}}} \int_{0}^{R} \int_{0}^{Z_{0}} \lambda_{2} \frac{\partial f_{2}}{\partial w_{r r}} \delta w_{r r} \mathrm{~d} z \mathrm{~d} r \mathrm{~d} t= & \int_{0}^{t_{\mathrm{f}}} \int_{0}^{Z_{0}}\left[\lambda_{2} \frac{\partial f_{2}}{\partial w_{r r}} \delta w_{r}\right]_{0}^{R} \mathrm{~d} z \mathrm{~d} t \\
& -\int_{0}^{t_{\mathrm{f}}} \int_{0}^{R} \int_{0}^{Z_{0}} \frac{\partial}{\partial r}\left(\lambda_{2} \frac{\partial f_{2}}{\partial w_{r r}}\right) \delta w_{r} \mathrm{~d} z \mathrm{~d} r \mathrm{~d} t .
\end{aligned}
$$


Integration by parts of the second integral of the previous equation with respect to $r$ results in

$$
\begin{aligned}
\int_{0}^{t_{\mathrm{f}}} \int_{0}^{R} \int_{0}^{Z_{0}} \lambda_{2} \frac{\partial f_{2}}{\partial w_{r r}} \delta w_{r r} \mathrm{~d} z \mathrm{~d} r \mathrm{~d} t= & \int_{0}^{t_{\mathrm{f}}} \int_{0}^{Z_{0}}\left[\lambda_{2} \frac{\partial f_{2}}{\partial w_{r r}} \delta w_{r}\right]_{0}^{R} \mathrm{~d} z \mathrm{~d} t \\
& -\int_{0}^{t_{\mathrm{f}}} \int_{0}^{Z_{0}}\left[\frac{\partial}{\partial r}\left(\lambda_{2} \frac{\partial f_{2}}{\partial w_{r r}}\right) \delta w\right]_{0}^{R} \mathrm{~d} z \mathrm{~d} t \\
& +\int_{0}^{t_{\mathrm{f}}} \int_{0}^{R} \int_{0}^{Z_{0}} \frac{\partial^{2}}{\partial r^{2}}\left(\lambda_{2} \frac{\partial f_{2}}{\partial w_{r r}}\right) \delta w \mathrm{~d} z \mathrm{~d} r \mathrm{~d} t .
\end{aligned}
$$

Considering that $\delta w$ is arbitrary at $r=R$ and the fact that all additive terms of $\delta J$ must be individually zero, it follows that

$$
\lambda_{2}(t, R, z)=0, \quad, \quad 0 \leq t \leq t_{\mathrm{f}} \text { and } 0 \leq z \leq Z_{0}
$$

Keeping in mind that $w_{r}$ is zero at $r=0$ because of symmetry around the $z$-axis and making use of these two conditions, previous expression gets simplified to

$$
\begin{aligned}
\int_{0}^{t_{\mathrm{f}}} \int_{0}^{R} \int_{0}^{Z_{0}} \lambda_{2} \frac{\partial f_{2}}{\partial w_{r r}} \delta w_{r r} \mathrm{~d} z \mathrm{~d} r \mathrm{~d} t= & -\int_{0}^{t_{\mathrm{f}}} \int_{0}^{Z_{0}}\left[\frac{\partial}{\partial r}\left(\lambda_{2} \frac{\partial f_{2}}{\partial w_{r r}}\right) \delta w\right]_{R} \mathrm{~d} z \mathrm{~d} t \\
& +\int_{0}^{t_{\mathrm{f}}} \int_{0}^{R} \int_{0}^{Z_{0}} \frac{\partial^{2}}{\partial r^{2}}\left(\lambda_{2} \frac{\partial f_{2}}{\partial w_{r r}}\right) \delta w \mathrm{~d} z \mathrm{~d} r \mathrm{~d} t .
\end{aligned}
$$

Integration by parts on the twenty third integral with respect to $r$ of equation G.4. we obtain

$$
\begin{aligned}
\int_{0}^{t_{\mathrm{f}}} \int_{0}^{R} \int_{0}^{Z_{0}} \lambda_{2} \frac{\partial f_{2}}{\partial T_{r r}} \delta T_{r r} \mathrm{~d} z \mathrm{~d} r \mathrm{~d} t= & \int_{0}^{t_{\mathrm{f}}} \int_{0}^{Z_{0}}\left[\lambda_{2} \frac{\partial f_{2}}{\partial T_{r r}} \delta T_{r}\right]_{0}^{R} \mathrm{~d} z \mathrm{~d} t \\
& -\int_{0}^{t_{\mathrm{f}}} \int_{0}^{R} \int_{0}^{Z_{0}} \frac{\partial}{\partial r}\left(\lambda_{2} \frac{\partial f_{2}}{\partial T_{r r}}\right) \delta T_{r} \mathrm{~d} z \mathrm{~d} r \mathrm{~d} t .
\end{aligned}
$$


Integration by parts of the second integral of the previous equation with respect to $\mathrm{r}$ results in

$$
\begin{aligned}
\int_{0}^{t_{\mathrm{f}}} \int_{0}^{R} \int_{0}^{Z_{0}} \lambda_{2} \frac{\partial f_{2}}{\partial T_{r r}} \delta T_{r r} \mathrm{~d} z \mathrm{~d} r \mathrm{~d} t= & \int_{0}^{t_{\mathrm{f}}} \int_{0}^{Z_{0}}\left[\lambda_{2} \frac{\partial f_{2}}{\partial T_{r r}} \delta T_{r}\right]_{0}^{R} \mathrm{~d} z \mathrm{~d} t \\
& -\int_{0}^{t_{\mathrm{f}}} \int_{0}^{Z_{0}}\left[\frac{\partial}{\partial r}\left(\lambda_{2} \frac{\partial f_{2}}{\partial T_{r r}}\right) \delta T\right]_{0}^{R} \mathrm{~d} z \mathrm{~d} t \\
& +\int_{0}^{t_{\mathrm{f}}} \int_{0}^{R} \int_{0}^{Z_{0}} \frac{\partial^{2}}{\partial r^{2}}\left(\lambda_{2} \frac{\partial f_{2}}{\partial T_{r r}}\right) \delta T \mathrm{~d} z \mathrm{~d} r \mathrm{~d} t .
\end{aligned}
$$

Considering that $\delta T$ is arbitrary at $r=R$ and the fact that all additive terms of $\delta J$ must be individually zero, it follows that

$$
\lambda_{2}(t, R, z)=0, \quad 0 \leq t \leq t_{\mathrm{f}} \text { and } 0 \leq z \leq Z_{0}
$$

Keeping in mind that $T_{r}$ is zero at $r=0$ because of symmetry around the z-axis and making use of these two conditions, previous expression gets simplified to

$$
\begin{aligned}
\int_{0}^{t_{\mathrm{f}}} \int_{0}^{R} \int_{0}^{Z_{0}} \lambda_{2} \frac{\partial f_{2}}{\partial T_{r r}} \delta T_{r r} \mathrm{~d} z \mathrm{~d} r \mathrm{~d} t= & -\int_{0}^{t_{\mathrm{f}}} \int_{0}^{Z_{0}}\left[\frac{\partial}{\partial r}\left(\lambda_{2} \frac{\partial f_{2}}{\partial T_{r r}}\right) \delta T\right]_{R} \mathrm{~d} z \mathrm{~d} t \\
& +\int_{0}^{t_{\mathrm{f}}} \int_{0}^{R} \int_{0}^{Z_{0}} \frac{\partial^{2}}{\partial r^{2}}\left(\lambda_{2} \frac{\partial f_{2}}{\partial T_{r r}}\right) \delta T \mathrm{~d} z \mathrm{~d} r \mathrm{~d} t .
\end{aligned}
$$

Integration by parts on the twenty second integral with respect to $z$ of equation G.4. we obtain

$$
\begin{aligned}
\int_{0}^{t_{\mathrm{f}}} \int_{0}^{R} \int_{0}^{Z_{0}} \lambda_{2} \frac{\partial f_{2}}{\partial w_{z z}} \delta w_{z z} \mathrm{~d} z \mathrm{~d} r \mathrm{~d} t= & \int_{0}^{t_{\mathrm{f}}} \int_{0}^{R}\left[\lambda_{2} \frac{\partial f_{2}}{\partial w_{z z}} \delta w_{z}\right]_{0}^{Z_{0}} \mathrm{~d} r \mathrm{~d} t \\
& -\int_{0}^{t_{\mathrm{f}}} \int_{0}^{R} \int_{0}^{Z_{0}} \frac{\partial}{\partial z}\left(\lambda_{2} \frac{\partial f_{2}}{\partial w_{z z}}\right) \delta w_{z} \mathrm{~d} z \mathrm{~d} r \mathrm{~d} t .
\end{aligned}
$$


Integration by parts of the second integral of the previous equation with respect to $z$ results in

$$
\begin{aligned}
\int_{0}^{t_{\mathrm{f}}} \int_{0}^{R} \int_{0}^{Z_{0}} \lambda_{2} \frac{\partial f_{2}}{\partial w_{z z}} \delta w_{z z} \mathrm{~d} z \mathrm{~d} r \mathrm{~d} t= & \int_{0}^{t_{\mathrm{f}}} \int_{0}^{R}\left[\lambda_{2} \frac{\partial f_{2}}{\partial w_{z z}} \delta w_{z}\right]_{0}^{Z_{0}} \mathrm{~d} r \mathrm{~d} t \\
& -\int_{0}^{t_{\mathrm{f}}} \int_{0}^{R}\left[\frac{\partial}{\partial z}\left(\lambda_{2} \frac{\partial f_{2}}{\partial w_{z z}}\right) \delta w\right]_{0}^{Z_{0}} \mathrm{~d} r \mathrm{~d} t \\
& +\int_{0}^{t_{\mathrm{f}}} \int_{0}^{R} \int_{0}^{Z_{0}} \frac{\partial^{2}}{\partial z^{2}}\left(\lambda_{2} \frac{\partial f_{2}}{\partial w_{z z}}\right) \delta w \mathrm{~d} z \mathrm{~d} r \mathrm{~d} t .
\end{aligned}
$$

Integration by parts on the twenty fourth integral with respect to $z$ of equation G.4 we obtain

$$
\begin{aligned}
\int_{0}^{t_{\mathrm{f}}} \int_{0}^{R} \int_{0}^{Z_{0}} \lambda_{2} \frac{\partial f_{2}}{\partial T_{z z}} \delta T_{z z} \mathrm{~d} z \mathrm{~d} r \mathrm{~d} t= & \int_{0}^{t_{\mathrm{f}}} \int_{0}^{R}\left[\lambda_{2} \frac{\partial f_{2}}{\partial T_{z z}} \delta T_{z}\right]_{0}^{Z_{0}} \mathrm{~d} r \mathrm{~d} t \\
& -\int_{0}^{t_{\mathrm{f}}} \int_{0}^{R} \int_{0}^{Z_{0}} \frac{\partial}{\partial z}\left(\lambda_{2} \frac{\partial f_{2}}{\partial T_{z z}}\right) \delta T_{z} \mathrm{~d} z \mathrm{~d} r \mathrm{~d} t .
\end{aligned}
$$

Integration by parts of the second integral of the previous equation with respect to $z$ results in

$$
\begin{aligned}
\int_{0}^{t_{\mathrm{f}}} \int_{0}^{R} \int_{0}^{Z_{0}} \lambda_{2} \frac{\partial f_{2}}{\partial T_{z z}} \delta T_{z z} \mathrm{~d} z \mathrm{~d} r \mathrm{~d} t= & \int_{0}^{t_{\mathrm{f}}} \int_{0}^{R}\left[\lambda_{2} \frac{\partial f_{2}}{\partial T_{z z}} \delta T_{z}\right]_{0}^{Z_{0}} \mathrm{~d} r \mathrm{~d} t \\
& -\int_{0}^{t_{\mathrm{f}}} \int_{0}^{R}\left[\frac{\partial}{\partial z}\left(\lambda_{2} \frac{\partial f_{2}}{\partial T_{z z}}\right) \delta T\right]_{0}^{Z_{0}} \mathrm{~d} r \mathrm{~d} t \\
& +\int_{0}^{t_{\mathrm{f}}} \int_{0}^{R} \int_{0}^{Z_{0}} \frac{\partial^{2}}{\partial z^{2}}\left(\lambda_{2} \frac{\partial f_{2}}{\partial T_{z z}}\right) \delta T \mathrm{~d} z \mathrm{~d} r \mathrm{~d} t .
\end{aligned}
$$


Simplification of the twenty fifth term yields

$$
\begin{aligned}
\frac{\lambda_{3}}{Z_{0}} \frac{\partial f_{3}}{\partial \bar{w}} \delta \bar{w}+\frac{\lambda_{3}}{Z_{0}} \frac{\partial f_{3}}{\partial \bar{T}} \delta \bar{T}= & \frac{\lambda_{3}}{Z_{0}} \frac{\partial f_{3}}{\partial \bar{w}}\left(\frac{\partial \bar{w}}{\partial w_{\text {int }}}\right) \delta w_{\text {int }}+\frac{\lambda_{3}}{Z_{0}} \frac{\partial f_{3}}{\partial \bar{T}}\left(\frac{\partial \bar{T}}{\partial T_{\text {int }}}\right) \delta T_{\text {int }} \\
& +\frac{\lambda_{3}}{Z_{0}} \frac{\partial f_{3}}{\partial \bar{w}}\left(\frac{\partial \bar{w}}{\partial w}\right) \delta w+\frac{\lambda_{3}}{Z_{0}} \frac{\partial f_{3}}{\partial \bar{T}}\left(\frac{\partial \bar{T}}{\partial T}\right) \delta T .
\end{aligned}
$$

It is noteworthy that

$$
\begin{aligned}
& \delta w(t, R, z)=\delta w(t, r, 0)=\delta w\left(t, r, Z_{0}\right)=\delta w_{\text {int }} ; \text { and } \\
& \delta T(t, R, z)=\delta T(t, r, 0)=\delta T\left(t, r, Z_{0}\right)=\delta T_{\text {int }}
\end{aligned}
$$

where $\delta w_{\text {int }}$ is the time-dependent mass fraction of nitrogen gas at the solvent-heavy oil interface and its dependence on the control function $T_{\text {int }}$ at a given $P$ is given by

$$
\delta w_{\mathrm{int}}=\left(\frac{\partial w_{\mathrm{int}}}{\partial T_{\mathrm{int}}}\right) \delta T_{\mathrm{int}} .
$$

Finally, from term one we obtain

$$
\begin{aligned}
\delta I= & -\frac{\beta r}{Z_{0} \bar{\mu}^{2}}\left[\frac{\partial \bar{\mu}}{\partial \bar{w}}\left(\frac{\partial \bar{w}}{\partial w_{\mathrm{int}}}\right)\left(\frac{\partial w_{\mathrm{int}}}{\partial T_{\mathrm{int}}}\right)+\frac{\partial \bar{\mu}}{\partial \bar{T}}\left(\frac{\partial \bar{T}}{\partial T_{\mathrm{int}}}\right)\right] \delta T_{\mathrm{int}} \\
& -\frac{\beta r}{Z_{0} \bar{\mu}^{2}}\left[\frac{\partial \bar{\mu}}{\partial \bar{w}}\left(\frac{\partial \bar{w}}{\partial w}\right) \delta w+\frac{\partial \bar{\mu}}{\partial \bar{T}}\left(\frac{\partial \bar{T}}{\partial T}\right) \delta T\right] .
\end{aligned}
$$

Also,

$$
\frac{\partial f_{3}}{\partial \bar{w}}=\frac{\alpha}{\phi \bar{\mu}^{2}} \frac{\partial \bar{\mu}}{\partial \bar{w}}
$$

and

$$
\frac{\partial f_{3}}{\partial \bar{T}}=\frac{\alpha}{\phi \bar{\mu}^{2}} \frac{\partial \bar{\mu}}{\partial \bar{T}}
$$


With the help of the aforementioned results, Equation G.4 can be re-expressed as

$$
\begin{aligned}
\delta J= & \int_{0}^{t_{\mathrm{f}}} \int_{0}^{R} \int_{0}^{Z_{0}}\left(\frac{\partial \lambda_{1}}{\partial t}+\lambda_{1} \frac{\partial f_{1}}{\partial T}+\lambda_{2} \frac{\partial f_{2}}{\partial T}-\frac{\partial}{\partial r}\left(\lambda_{1} \frac{\partial f_{1}}{\partial T_{r}}\right)-\frac{\partial}{\partial z}\left(\lambda_{1} \frac{\partial f_{1}}{\partial T_{z}}\right)\right. \\
& -\frac{\partial}{\partial r}\left(\lambda_{2} \frac{\partial f_{2}}{\partial T_{r}}\right)-\frac{\partial}{\partial z}\left(\lambda_{2} \frac{\partial f_{2}}{\partial T_{z}}\right)+\frac{\partial^{2}}{\partial r^{2}}\left(\lambda_{1} \frac{\partial f_{1}}{\partial T_{r r}}\right)+\frac{\partial^{2}}{\partial z^{2}}\left(\lambda_{1} \frac{\partial f_{1}}{\partial T_{z z}}\right) \\
& \left.+\frac{\partial^{2}}{\partial r^{2}}\left(\lambda_{2} \frac{\partial f_{2}}{\partial T_{r r}}\right)+\frac{\partial^{2}}{\partial z^{2}}\left(\lambda_{2} \frac{\partial f_{2}}{\partial T_{z z}}\right)-\left(2 \pi r \rho-\frac{\lambda_{3}}{\phi}\right) \frac{\alpha}{Z_{0} \bar{\mu}^{2}} \frac{\partial \bar{\mu}}{\partial \bar{T}} \frac{\partial \bar{T}}{\partial T}\right) \delta T \mathrm{~d} z \mathrm{~d} r \mathrm{~d} t \\
& +\int_{0}^{t_{\mathrm{f}}} \int_{0}^{R} \int_{0}^{Z_{0}}\left(\frac{\partial \lambda_{2}}{\partial t}+\lambda_{1} \frac{\partial f_{1}}{\partial w}+\lambda_{2} \frac{\partial f_{2}}{\partial w}-\frac{\partial}{\partial r}\left(\lambda_{1} \frac{\partial f_{1}}{\partial w_{r}}\right)-\frac{\partial}{\partial z}\left(\lambda_{1} \frac{\partial f_{1}}{\partial w_{z}}\right)\right. \\
& -\frac{\partial}{\partial r}\left(\lambda_{2} \frac{\partial f_{2}}{\partial w_{r}}\right)-\frac{\partial}{\partial z}\left(\lambda_{2} \frac{\partial f_{2}}{\partial w_{z}}\right)+\frac{\partial^{2}}{\partial r^{2}}\left(\lambda_{1} \frac{\partial f_{1}}{\partial w_{r r}}\right)+\frac{\partial^{2}}{\partial z^{2}}\left(\lambda_{1} \frac{\partial f_{1}}{\partial w_{z z}}\right) \\
& \left.+\frac{\partial^{2}}{\partial r^{2}}\left(\lambda_{2} \frac{\partial f_{2}}{\partial w_{r r}}\right)+\frac{\partial^{2}}{\partial z^{2}}\left(\lambda_{2} \frac{\partial f_{2}}{\partial w_{z z}}\right)-\left(2 \pi r \rho-\frac{\lambda_{3}}{\phi}\right) \frac{\alpha}{Z_{0} \bar{\mu}^{2}} \frac{\partial \bar{\mu}}{\partial \bar{w}} \frac{\partial \bar{w}}{\partial w}\right) \delta w \mathrm{~d} z \mathrm{~d} r \mathrm{~d} t \\
& +\int_{0}^{t_{\mathrm{f}}} \int_{0}^{R} \int_{0}^{Z_{0}}\left(\frac{1}{Z_{0}} \times \frac{\partial \lambda_{3}}{\partial t}\right) \delta z \mathrm{~d} z \mathrm{~d} r \mathrm{~d} t+\int_{0}^{t_{\mathrm{f}}} \int_{0}^{R} \int_{0}^{Z_{0}}(X) \delta T_{\text {int }} \mathrm{d} z \mathrm{~d} r \mathrm{~d} t \\
+ & \int_{0}^{t_{\mathrm{f}}} \int_{0}^{R} \int_{0}^{Z_{0}}\left(G_{1} \delta \lambda_{1}+G_{2} \delta \lambda_{2}+\frac{G_{3}}{Z_{0}} \delta \lambda_{3}\right) \mathrm{d} z \mathrm{~d} r \mathrm{~d} t
\end{aligned}
$$


where,

$$
\begin{aligned}
& X=\left\{\frac{1}{R}\left[\lambda_{1} \frac{\partial f_{1}}{\partial w_{r}}\right]_{R}\left(\frac{\partial w_{\mathrm{int}}}{\partial T_{\mathrm{int}}}\right)+\frac{1}{Z_{0}}\left[\lambda_{1} \frac{\partial f_{1}}{\partial w_{z}}\right]_{Z_{0}}\left(\frac{\partial w_{\mathrm{int}}}{\partial T_{\mathrm{int}}}\right)-\frac{1}{Z_{0}}\left[\lambda_{1} \frac{\partial f_{1}}{\partial w_{z}}\right]_{0}\left(\frac{\partial w_{\mathrm{int}}}{\partial T_{\mathrm{int}}}\right)\right. \\
& +\frac{1}{R}\left[\lambda_{1} \frac{\partial f_{1}}{\partial T_{r}}\right]_{R}+\frac{1}{Z_{0}}\left[\lambda_{1} \frac{\partial f_{1}}{\partial T_{z}}\right]_{Z_{0}}-\frac{1}{Z_{0}}\left[\lambda_{1} \frac{\partial f_{1}}{\partial T_{z}}\right]_{0}+\frac{1}{Z_{0}}\left[\lambda_{1} \frac{\partial f_{1}}{\partial w_{z z}} \delta w_{z}\right]_{0}^{Z_{0}} \\
& -\frac{1}{R}\left[\frac{\partial}{\partial r}\left(\lambda_{1} \frac{\partial f_{1}}{\partial w_{r r}}\right)\right]_{R}\left(\frac{\partial w_{\text {int }}}{\partial T_{\text {int }}}\right)-\frac{1}{R}\left[\frac{\partial}{\partial r}\left(\lambda_{1} \frac{\partial f_{1}}{\partial T_{r r}}\right)\right]_{R}+\frac{1}{Z_{0}}\left[\lambda_{1} \frac{\partial f_{1}}{\partial T_{z z}} \delta T_{z}\right]_{0}^{Z_{0}} \\
& -\frac{1}{Z_{0}}\left[\frac{\partial}{\partial z}\left(\lambda_{1} \frac{\partial f_{1}}{\partial w_{z z}}\right)\right]_{Z_{0}}\left(\frac{\partial w_{\mathrm{int}}}{\partial T_{\mathrm{int}}}\right)+\frac{1}{Z_{0}}\left[\frac{\partial}{\partial z}\left(\lambda_{1} \frac{\partial f_{1}}{\partial w_{z z}}\right)\right]_{0}\left(\frac{\partial w_{\mathrm{int}}}{\partial T_{\mathrm{int}}}\right) \\
& -\frac{1}{Z_{0}}\left[\frac{\partial}{\partial z}\left(\lambda_{1} \frac{\partial f_{1}}{\partial T_{z z}}\right)\right]_{Z_{0}}+\frac{1}{Z_{0}}\left[\frac{\partial}{\partial z}\left(\lambda_{1} \frac{\partial f_{1}}{\partial T_{z z}}\right)\right]_{0} \\
& +\frac{1}{R}\left[\lambda_{2} \frac{\partial f_{2}}{\partial w_{r}}\right]_{R}\left(\frac{\partial w_{\mathrm{int}}}{\partial T_{\mathrm{int}}}\right)+\frac{1}{Z_{0}}\left[\lambda_{2} \frac{\partial f_{2}}{\partial w_{z}}\right]_{Z_{0}}\left(\frac{\partial w_{\mathrm{int}}}{\partial T_{\mathrm{int}}}\right)-\frac{1}{Z_{0}}\left[\lambda_{2} \frac{\partial f_{2}}{\partial w_{z}}\right]_{0}\left(\frac{\partial w_{\mathrm{int}}}{\partial T_{\mathrm{int}}}\right) \\
& +\frac{1}{R}\left[\lambda_{2} \frac{\partial f_{2}}{\partial T_{r}}\right]_{R}+\frac{1}{Z_{0}}\left[\lambda_{2} \frac{\partial f_{2}}{\partial T_{z}}\right]_{Z_{0}}-\frac{1}{Z_{0}}\left[\lambda_{2} \frac{\partial f_{2}}{\partial T_{z}}\right]_{0}+\frac{1}{Z_{0}}\left[\lambda_{2} \frac{\partial f_{2}}{\partial w_{z z}} \delta w_{z}\right]_{0}^{Z_{0}} \\
& -\frac{1}{R}\left[\frac{\partial}{\partial r}\left(\lambda_{2} \frac{\partial f_{2}}{\partial w_{r r}}\right)\right]_{R}\left(\frac{\partial w_{\text {int }}}{\partial T_{\text {int }}}\right)-\frac{1}{R}\left[\frac{\partial}{\partial r}\left(\lambda_{2} \frac{\partial f_{2}}{\partial T_{r r}}\right)\right]_{R}+\frac{1}{Z_{0}}\left[\lambda_{2} \frac{\partial f_{2}}{\partial T_{z z}} \delta T_{z}\right]_{0}^{Z_{0}} \\
& -\frac{1}{Z_{0}}\left[\frac{\partial}{\partial z}\left(\lambda_{2} \frac{\partial f_{2}}{\partial w_{z z}}\right)\right]_{Z_{0}}\left(\frac{\partial w_{\text {int }}}{\partial T_{\text {int }}}\right)+\frac{1}{Z_{0}}\left[\frac{\partial}{\partial z}\left(\lambda_{2} \frac{\partial f_{2}}{\partial w_{z z}}\right)\right]_{0}\left(\frac{\partial w_{\text {int }}}{\partial T_{\text {int }}}\right) \\
& -\frac{1}{Z_{0}}\left[\frac{\partial}{\partial z}\left(\lambda_{2} \frac{\partial f_{2}}{\partial T_{z z}}\right)\right]_{Z_{0}}+\frac{1}{Z_{0}}\left[\frac{\partial}{\partial z}\left(\lambda_{2} \frac{\partial f_{2}}{\partial T_{z z}}\right)\right]_{0}+\frac{\lambda_{3}}{Z_{0}} \frac{\partial f_{3}}{\partial \bar{w}}\left(\frac{\partial \bar{w}}{\partial w_{\mathrm{int}}}\right)\left(\frac{\partial w_{\mathrm{int}}}{\partial T_{\mathrm{int}}}\right) \\
& \left.+\frac{\lambda_{3}}{Z_{0}} \frac{\partial f_{3}}{\partial \bar{T}}\left(\frac{\partial \bar{T}}{\partial T_{\mathrm{int}}}\right)-\frac{\beta r}{Z_{0} \cdot \bar{\mu}^{2}}\left[\frac{\partial \bar{\mu}}{\partial \bar{w}}\left(\frac{\partial \bar{w}}{\partial w_{\mathrm{int}}}\right)\left(\frac{\partial w_{\mathrm{int}}}{\partial T_{\mathrm{int}}}\right)+\frac{\partial \bar{\mu}}{\partial \bar{T}}\left(\frac{\partial \bar{T}}{\partial T_{\mathrm{int}}}\right)\right]\right\} .
\end{aligned}
$$

In addition, $\delta w$ and $\delta T$ is arbitrary at $z=0 \& Z_{0}$ and the fact that all additive terms 
of $\delta J$ must be individually zero, it follows that

$$
\begin{gathered}
\lambda_{1}(t, r, 0)=0, \quad \lambda_{1}\left(t, r, Z_{0}\right)=0, \quad \lambda_{2}(t, r, 0)=0, \quad \lambda_{2}\left(t, r, Z_{0}\right)=0, \\
0 \leq t \leq t_{\mathrm{f}} \text { and } 0 \leq r \leq R
\end{gathered}
$$

In the context of Equation G.61 the coefficients of $\delta T, \delta w, \delta z, \delta \lambda_{1}, \delta \lambda_{2}, \delta \lambda_{1}$ and $\delta T_{\text {int }}$ as indicated earlier expression depend on the arbitrary variations these terms respectively, and they are assumed to be continuous. Under these conditions, for $\delta J$ to be zero, each of these coefficients must be individually zero. As a result, we obtain by necessity

Co-state Equation 1:

$$
\begin{aligned}
\frac{\partial \lambda_{1}}{\partial t}= & -\lambda_{1} \frac{\partial f_{1}}{\partial T}-\lambda_{2} \frac{\partial f_{2}}{\partial T}+\frac{\partial}{\partial r}\left(\lambda_{1} \frac{\partial f_{1}}{\partial T_{r}}\right)+\frac{\partial}{\partial z}\left(\lambda_{1} \frac{\partial f_{1}}{\partial T_{z}}\right)+\frac{\partial}{\partial r}\left(\lambda_{2} \frac{\partial f_{2}}{\partial T_{r}}\right) \\
& +\frac{\partial}{\partial z}\left(\lambda_{2} \frac{\partial f_{2}}{\partial T_{z}}\right)-\frac{\partial^{2}}{\partial r^{2}}\left(\lambda_{1} \frac{\partial f_{1}}{\partial T_{r r}}\right)-\frac{\partial^{2}}{\partial z^{2}}\left(\lambda_{1} \frac{\partial f_{1}}{\partial T_{z z}}\right)-\frac{\partial^{2}}{\partial r^{2}}\left(\lambda_{2} \frac{\partial f_{2}}{\partial T_{r r}}\right) \\
& -\frac{\partial^{2}}{\partial z^{2}}\left(\lambda_{2} \frac{\partial f_{2}}{\partial T_{z z}}\right)+\left(2 \pi r \rho-\frac{\lambda_{3}}{\phi}\right) \frac{\alpha}{Z_{0} \bar{\mu}^{2}} \frac{\partial \bar{\mu}}{\partial \bar{T}} \frac{\partial \bar{T}}{\partial T},
\end{aligned}
$$

and $0 \leq r \leq R$ and $0 \leq z \leq Z_{0}$

Co-state Equation 2:

$$
\begin{aligned}
\frac{\partial \lambda_{2}}{\partial t}= & -\lambda_{1} \frac{\partial f_{1}}{\partial w}-\lambda_{2} \frac{\partial f_{2}}{\partial w}+\frac{\partial}{\partial r}\left(\lambda_{1} \frac{\partial f_{1}}{\partial w_{r}}\right)+\frac{\partial}{\partial z}\left(\lambda_{1} \frac{\partial f_{1}}{\partial w_{z}}\right)+\frac{\partial}{\partial r}\left(\lambda_{2} \frac{\partial f_{2}}{\partial w_{r}}\right) \\
& +\frac{\partial}{\partial z}\left(\lambda_{2} \frac{\partial f_{2}}{\partial w_{z}}\right)-\frac{\partial^{2}}{\partial r^{2}}\left(\lambda_{1} \frac{\partial f_{1}}{\partial w_{r r}}\right)-\frac{\partial^{2}}{\partial z^{2}}\left(\lambda_{1} \frac{\partial f_{1}}{\partial w_{z z}}\right)-\frac{\partial^{2}}{\partial r^{2}}\left(\lambda_{2} \frac{\partial f_{2}}{\partial w_{r r}}\right) \\
& -\frac{\partial^{2}}{\partial z^{2}}\left(\lambda_{2} \frac{\partial f_{2}}{\partial w_{z z}}\right)+\left(2 \pi r \rho-\frac{\lambda_{3}}{\phi}\right) \frac{\alpha}{Z_{0} \bar{\mu}^{2}} \frac{\partial \bar{\mu}}{\partial \bar{w}} \frac{\partial \bar{w}}{\partial w},
\end{aligned}
$$

and $0 \leq r \leq R$ and $0 \leq z \leq Z_{0}$

Co-state Equation 3:

$$
\begin{aligned}
& \frac{\partial \lambda_{3}}{\partial t}=0, \\
& \quad \text { and } 0 \leq r \leq R
\end{aligned}
$$


Stationarity Equation:

$$
\begin{aligned}
\frac{\partial J}{\partial T_{\mathrm{int}}}= & -\frac{1}{R}\left[\frac{\partial}{\partial r}\left(\lambda_{1} \frac{\partial f_{1}}{\partial w_{r r}}\right)\right]_{R}\left(\frac{\partial w_{\mathrm{int}}}{\partial T_{\mathrm{int}}}\right)-\frac{1}{R}\left[\frac{\partial}{\partial r}\left(\lambda_{1} \frac{\partial f_{1}}{\partial T_{r r}}\right)\right]_{R} \\
& -\frac{1}{Z_{0}}\left[\frac{\partial}{\partial z}\left(\lambda_{1} \frac{\partial f_{1}}{\partial w_{z z}}\right)\right]_{Z_{0}}\left(\frac{\partial w_{\mathrm{int}}}{\partial T_{\mathrm{int}}}\right)+\frac{1}{Z_{0}}\left[\frac{\partial}{\partial z}\left(\lambda_{1} \frac{\partial f_{1}}{\partial w_{z z}}\right)\right]_{0}\left(\frac{\partial w_{\mathrm{int}}}{\partial T_{\mathrm{int}}}\right) \\
& -\frac{1}{Z_{0}}\left[\frac{\partial}{\partial z}\left(\lambda_{1} \frac{\partial f_{1}}{\partial T_{z z}}\right)\right]_{Z_{0}}+\frac{1}{Z_{0}}\left[\frac{\partial}{\partial z}\left(\lambda_{1} \frac{\partial f_{1}}{\partial T_{z z}}\right)\right]_{0} \\
& -\frac{1}{R}\left[\frac{\partial}{\partial r}\left(\lambda_{2} \frac{\partial f_{2}}{\partial w_{r r}}\right)\right]_{R}\left(\frac{\partial w_{\mathrm{int}}}{\partial T_{\mathrm{int}}}\right)-\frac{1}{R}\left[\frac{\partial}{\partial r}\left(\lambda_{2} \frac{\partial f_{2}}{\partial T_{r r}}\right)\right]_{R} \\
& -\frac{1}{Z_{0}}\left[\frac{\partial}{\partial z}\left(\lambda_{2} \frac{\partial f_{2}}{\partial w_{z z}}\right)\right]_{Z_{0}}\left(\frac{\partial w_{\mathrm{int}}}{\partial T_{\mathrm{int}}}\right)+\frac{1}{Z_{0}}\left[\frac{\partial}{\partial z}\left(\lambda_{2} \frac{\partial f_{2}}{\partial w_{z z}}\right)\right]_{0}\left(\frac{\partial w_{\mathrm{int}}}{\partial T_{\mathrm{int}}}\right) \\
& -\frac{1}{Z_{0}}\left[\frac{\partial}{\partial z}\left(\lambda_{2} \frac{\partial f_{2}}{\partial T_{z z}}\right)\right]_{Z_{0}}+\frac{1}{Z_{0}}\left[\frac{\partial}{\partial z}\left(\lambda_{2} \frac{\partial f_{2}}{\partial T_{z z}}\right)\right]_{0}+\frac{\lambda_{3}}{Z_{0}} \frac{\partial f_{3}}{\partial \bar{w}}\left(\frac{\partial \bar{w}}{\partial w_{\mathrm{int}}}\right)\left(\frac{\partial w_{\mathrm{int}}}{\partial T_{\mathrm{int}}}\right) \\
+ & \left.\frac{\lambda_{3}}{Z_{0}} \frac{\partial f_{3}}{\partial \bar{T}}\left(\frac{\partial \bar{T}}{\partial T_{\mathrm{int}}}\right)-\frac{\partial \bar{\mu}}{Z_{0} \cdot \bar{\mu}^{2}}\left[\frac{\partial \bar{w}}{\partial \bar{w}}\left(\frac{\partial w_{\mathrm{int}}}{\partial w_{\mathrm{int}}}\right)+\frac{\partial \bar{\mu}}{\partial \bar{T}}\left(\frac{\partial \bar{T}}{\partial T_{\mathrm{int}}}\right)\right]\right\} .
\end{aligned}
$$

From Equation G.64, if we simplify different terms, we obtain following relations:

$$
\begin{aligned}
\frac{\partial f_{1}}{\partial T}= & -\frac{\alpha}{\phi \mu^{2}}\left(\mu_{w} w_{z}+\mu_{T} T_{z}\right)+\frac{2 \alpha T \mu_{T}}{\phi \mu^{3}}\left(\mu_{w} w_{z}+\mu_{T} T_{z}\right)-\frac{\alpha T_{z} \mu_{T}}{\phi \mu^{2}} \\
& -\frac{\alpha T}{\phi \mu^{2}}\left(\mu_{w T} w_{z}+\mu_{T T} T_{z}\right) \\
\frac{\partial f_{2}}{\partial T}= & \frac{D_{T} w_{r}}{r}+D_{T} w_{r r}+D_{w T}\left(w_{r}\right)^{2}+D_{T T} w_{r} T_{r}-\frac{\alpha \mu_{T} w_{z}}{\phi \mu^{2}} \\
& +\frac{2 \alpha w \mu_{T}}{\phi \mu^{3}}\left(\mu_{w} w_{z}+\mu_{T} T_{z}\right)-\frac{\alpha w}{\phi \mu^{2}}\left(\mu_{w T} w_{z}+\mu_{T T} T_{z}\right) ;
\end{aligned}
$$

From the derivatives of $f_{1}$ with respect to derivatives of $T, w$ along $r$ and $z$,

$$
\frac{\partial f_{1}}{\partial T_{r}}=\frac{\gamma}{r}
$$




$$
\begin{aligned}
& \frac{\partial f_{1}}{\partial T_{z}}=\frac{\alpha}{\phi \mu}-\frac{\alpha T \mu_{T}}{\phi \mu^{2}} \\
& \frac{\partial f_{1}}{\partial T_{r r}}=\gamma \\
& \frac{\partial f_{1}}{\partial T_{z z}}=\gamma \\
& \frac{\partial f_{2}}{\partial T_{r}}=D_{T} w_{r} \\
& \frac{\partial f_{2}}{\partial T_{z}}=-\frac{\alpha w \mu_{T}}{\phi \mu^{2}} \\
& \frac{\partial f_{2}}{\partial T_{r r}}=0 \\
& \frac{\partial f_{2}}{\partial T_{z z}}=0
\end{aligned}
$$

Similarly, from Equation G.65, if we simplify different terms, we obtain following relations:

$$
\begin{aligned}
\frac{\partial f_{1}}{\partial w}= & \frac{2 \alpha T \mu_{w}}{\phi \mu^{3}}\left(\mu_{w} w_{z}+\mu_{T} T_{z}\right)-\frac{\alpha T_{z} \mu_{w}}{\phi \mu^{2}}-\frac{\alpha T}{\phi \mu^{2}}\left(\mu_{w w} w_{z}+\mu_{w T} T_{z}\right) \\
\frac{\partial f_{2}}{\partial w}= & \frac{D_{w} w_{r}}{r}+D_{w} w_{r r}+D_{w w}\left(w_{r}\right)^{2}+D_{w T} w_{r} T_{r}-\frac{\alpha}{\phi \mu^{2}}\left(\mu_{w} w_{z}+\mu_{T} T_{z}\right) \\
& +\frac{2 \alpha w \mu_{w}}{\phi \mu^{3}}\left(\mu_{w} w_{z}+\mu_{T} T_{z}\right)-\frac{\alpha w}{\phi \mu^{2}}\left(\mu_{w w} w_{z}+\mu_{w T} T_{z}\right)-\frac{\alpha w_{z} \mu_{w}}{\phi \mu^{2}} \\
\frac{\partial f_{1}}{\partial w_{r}}= & 0 \\
\frac{\partial f_{1}}{\partial w_{z}}= & -\frac{\alpha T \mu_{w}}{\phi \mu^{2}} \\
\frac{\partial f_{1}}{\partial w_{r r}}= & 0 \\
\frac{\partial f_{1}}{\partial w_{z z}}= & 0 \\
\frac{\partial f_{2}}{\partial w_{r}}= & \frac{D}{r}+2 D_{w} w_{r}+D_{T} T_{r} \\
\frac{\partial f_{2}}{\partial w_{z}}= & \frac{\alpha}{\phi \mu}-\frac{\alpha w \mu_{w}}{\phi \mu^{2}}
\end{aligned}
$$




$$
\begin{aligned}
& \frac{\partial f_{2}}{\partial w_{r r}}=D \\
& \frac{\partial f_{2}}{\partial w_{z z}}=0 .
\end{aligned}
$$

Moreover, from the double derivatives,

$$
\begin{aligned}
& \frac{\partial}{\partial r}\left(\lambda_{1} \frac{\partial f_{1}}{\partial T_{r}}\right)=\left(\frac{\gamma}{r}\right) \frac{\partial \lambda_{1}}{\partial r}+\lambda_{1} \frac{\partial}{\partial r}\left(\frac{\partial f_{1}}{\partial T_{r}}\right)=\left(\frac{\gamma}{r}\right) \frac{\partial \lambda_{1}}{\partial r}+\lambda_{1} \frac{\partial}{\partial r}\left(\frac{\gamma}{r}\right) \\
& =\left(\frac{\gamma}{r}\right) \frac{\partial \lambda_{1}}{\partial r}-\frac{\gamma \lambda_{1}}{r^{2}} \\
& \frac{\partial}{\partial z}\left(\lambda_{1} \frac{\partial f_{1}}{\partial T_{z}}\right)=\left(\frac{\partial f_{1}}{\partial T_{z}}\right) \frac{\partial \lambda_{1}}{\partial z}+\frac{\partial}{\partial z}\left(\frac{\partial f_{1}}{\partial T_{z}}\right) \\
& =\left(\frac{\alpha}{\phi \mu}-\frac{\alpha T \mu_{T}}{\phi \mu^{2}}\right) \frac{\partial \lambda_{1}}{\partial z}-\frac{\alpha \lambda_{1}}{\phi \mu^{2}}\left(\mu_{w} w_{z}+\mu_{T} T_{z}\right)-\frac{\alpha \mu_{T} T_{z} \lambda_{1}}{\phi \mu^{2}} \\
& -\frac{\alpha T \lambda_{1}}{\phi \mu^{2}}\left(\mu_{w T} w_{z}+\mu_{T T} T_{z}\right)+\frac{2 \alpha T \mu_{T} \lambda_{1}}{\phi \mu^{3}}\left(\mu_{w} w_{z}+\mu_{T} T_{z}\right) \\
& \frac{\partial}{\partial r}\left(\lambda_{2} \frac{\partial f_{2}}{\partial T_{r}}\right)=\left(\frac{\partial f_{2}}{\partial T_{r}}\right) \frac{\partial \lambda_{2}}{\partial r}+\lambda_{2} \frac{\partial}{\partial r}\left(\frac{\partial f_{2}}{\partial T_{r}}\right) \\
& =D_{T} w_{r} \frac{\partial \lambda_{2}}{\partial r}+\lambda_{2}\left(D_{T} w_{r r}+D_{w T}\left(w_{r}\right)^{2}+D_{T T} w_{r} T_{r}\right) \\
& \frac{\partial}{\partial z}\left(\lambda_{2} \frac{\partial f_{2}}{\partial T_{z}}\right)=\left(\frac{\partial f_{2}}{\partial T_{z}}\right) \frac{\partial \lambda_{2}}{\partial z}+\lambda_{2} \frac{\partial}{\partial z}\left(\frac{\partial f_{2}}{\partial T_{z}}\right) \\
& =-\left(\frac{\alpha w \mu_{T}}{\phi \mu^{2}}\right) \frac{\partial \lambda_{2}}{\partial z}-\frac{\alpha \mu_{T} w_{z} \lambda_{2}}{\phi \mu^{2}}-\frac{\alpha w \lambda_{2}}{\phi \mu^{2}}\left(\mu_{w T} w_{z}+\mu_{T T} T_{z}\right) \\
& +\frac{2 \alpha w \mu_{T} \lambda_{2}}{\phi \mu^{3}}\left(\mu_{w} w_{z}+\mu_{T} T_{z}\right) \\
& \frac{\partial}{\partial r}\left(\lambda_{1} \frac{\partial f_{1}}{\partial w_{r}}\right)=0
\end{aligned}
$$




$$
\begin{gathered}
\frac{\partial}{\partial z}\left(\lambda_{1} \frac{\partial f_{1}}{\partial w_{z}}\right)=\left(\frac{\partial f_{1}}{\partial w_{z}}\right) \frac{\partial \lambda_{1}}{\partial z}+\lambda_{1} \frac{\partial}{\partial z}\left(\frac{\partial f_{1}}{\partial w_{z}}\right) \\
=-\left(\frac{\alpha T \mu_{w}}{\phi \mu^{2}}\right) \frac{\partial \lambda_{1}}{\partial z}-\frac{\alpha \mu_{w} T_{z} \lambda_{1}}{\phi \mu^{2}}-\frac{\alpha T \lambda_{1}}{\phi \mu^{2}}\left(\mu_{w w} w_{z}+\mu_{w T} T_{z}\right) \\
+\frac{2 \alpha T \mu_{w} \lambda_{1}}{\phi \mu^{3}}\left(\mu_{w} w_{z}+\mu_{T} T_{z}\right) \\
\frac{\partial}{\partial r}\left(\lambda_{2} \frac{\partial f_{2}}{\partial w_{r}}\right)=\left(\frac{\partial f_{2}}{\partial w_{r}}\right) \frac{\partial \lambda_{2}}{\partial r}+\lambda_{2} \frac{\partial}{\partial r}\left(\frac{\partial f_{2}}{\partial w_{r}}\right)=\left(\frac{D}{r}+2 D_{w} w_{r}+D_{T} T_{r}\right) \frac{\partial \lambda_{2}}{\partial r} \\
-\frac{D \lambda_{2}}{r^{2}}+2 D_{w} w_{r r} \lambda_{2}+D_{T} T_{r r} \lambda_{2}+\frac{\lambda_{2}}{r}\left(D_{w} w_{r}+D_{T} T_{r}\right) \\
+2 \lambda_{2} w_{r}\left(D_{w w} w_{r}+D_{w T} T_{r}\right)+\lambda_{2} T_{r}\left(D_{w T} w_{r}+D_{T T} T_{r}\right)
\end{gathered}
$$

$$
\begin{gathered}
\frac{\partial}{\partial z}\left(\lambda_{2} \frac{\partial f_{2}}{\partial w_{z}}\right)=\left(\frac{\partial f_{2}}{\partial w_{z}}\right) \frac{\partial \lambda_{2}}{\partial z}+\lambda_{2} \frac{\partial}{\partial z}\left(\frac{\partial f_{2}}{\partial w_{z}}\right) \\
=\left(\frac{\alpha}{\phi \mu}-\frac{\alpha w \mu_{w}}{\phi \mu^{2}}\right) \frac{\partial \lambda_{2}}{\partial z}-\frac{\alpha \lambda_{2}}{\phi \mu^{2}}\left(\mu_{w} w_{z}+\mu_{T} T_{z}\right)-\frac{\alpha \mu_{w} w_{z} \lambda_{2}}{\phi \mu^{2}} \\
-\frac{\alpha w \lambda_{2}}{\phi \mu^{2}}\left(\mu_{w w} w_{z}+\mu_{w T} T_{z}\right)+\frac{2 \alpha w \mu_{w} \lambda_{2}}{\phi \mu^{3}}\left(\mu_{w} w_{z}+\mu_{T} T_{z}\right)
\end{gathered}
$$

$$
\begin{aligned}
\frac{\partial^{2}}{\partial r^{2}}\left(\lambda_{1} \frac{\partial f_{1}}{\partial T_{r r}}\right) & =\gamma \frac{\partial^{2} \lambda_{1}}{\partial r^{2}} \\
\frac{\partial^{2}}{\partial z^{2}}\left(\lambda_{1} \frac{\partial f_{1}}{\partial T_{z z}}\right) & =\gamma \frac{\partial^{2} \lambda_{1}}{\partial z^{2}} \\
\frac{\partial^{2}}{\partial r^{2}}\left(\lambda_{2} \frac{\partial f_{2}}{\partial T_{r r}}\right) & =0 \\
\frac{\partial^{2}}{\partial z^{2}}\left(\lambda_{2} \frac{\partial f_{2}}{\partial T_{z z}}\right) & =0
\end{aligned}
$$




$$
\begin{aligned}
\frac{\partial^{2}}{\partial r^{2}}\left(\lambda_{1} \frac{\partial f_{1}}{\partial w_{r r}}\right) & =0 \\
\frac{\partial^{2}}{\partial z^{2}}\left(\lambda_{1} \frac{\partial f_{1}}{\partial w_{z z}}\right) & =0 \\
\frac{\partial^{2}}{\partial r^{2}}\left(\lambda_{2} \frac{\partial f_{2}}{\partial w_{r r}}\right) & =D \frac{\partial^{2} \lambda_{2}}{\partial r^{2}}+\lambda_{2} \frac{\partial^{2}}{\partial r^{2}}\left(\frac{\partial f_{2}}{\partial w_{r r}}\right) \\
=D \frac{\partial^{2} \lambda_{2}}{\partial r^{2}} & +\lambda_{2}\left[D_{w} w_{r r}+D_{T} T_{r r}+D_{w w}\left(w_{r}\right)^{2}+D_{T T}\left(T_{r}\right)^{2}+2 D_{w T} w_{r} T_{r}\right]
\end{aligned}
$$

and,

$$
\frac{\partial^{2}}{\partial z^{2}}\left(\lambda_{2} \frac{\partial f_{2}}{\partial w_{z z}}\right)=0 .
$$

Using above relationships, we obtain final form of the first co-state equation as:

$$
\begin{aligned}
\frac{\partial \lambda_{1}}{\partial t}= & \frac{\gamma}{r} \frac{\partial \lambda_{1}}{\partial r}-\frac{\gamma \lambda_{1}}{r^{2}}-\gamma \frac{\partial^{2} \lambda_{1}}{\partial r^{2}}-\gamma \frac{\partial^{2} \lambda_{1}}{\partial z^{2}}+\frac{\alpha}{\phi \mu} \frac{\partial \lambda_{1}}{\partial z}+\frac{\partial D}{\partial T} \frac{\partial w}{\partial r} \frac{\partial \lambda_{2}}{\partial r}-\frac{\lambda_{2}}{r} \frac{\partial D}{\partial T} \frac{\partial w}{\partial r} \\
& -\frac{\alpha T}{\phi \mu^{2}} \frac{\partial \mu}{\partial T} \frac{\partial \lambda_{1}}{\partial z}-\frac{\alpha w}{\phi \mu^{2}} \frac{\partial \mu}{\partial T} \frac{\partial \lambda_{2}}{\partial z}+\left(2 \pi r \rho-\frac{\lambda_{3}}{\phi}\right) \frac{\alpha}{Z_{0} \bar{\mu}^{2}} \frac{\partial \bar{\mu}}{\partial \bar{T}} \frac{\partial \bar{T}}{\partial T}
\end{aligned}
$$

similarly, using above relationships, we obtain final form of the second co-state equation as:

$$
\begin{aligned}
\frac{\partial \lambda_{2}}{\partial t}= & \frac{D}{r} \frac{\partial \lambda_{2}}{\partial r}-\frac{D \lambda_{2}}{r^{2}}-D \frac{\partial^{2} \lambda_{2}}{\partial r^{2}}+\frac{\alpha}{\phi \mu} \frac{\partial \lambda_{2}}{\partial z}+\frac{\partial D}{\partial T} \frac{\partial T}{\partial r} \frac{\partial \lambda_{2}}{\partial r}+\frac{\lambda_{2}}{r} \frac{\partial D}{\partial T} \frac{\partial T}{\partial r} \\
& +2 \frac{\partial D}{\partial w} \frac{\partial w}{\partial r} \frac{\partial \lambda_{2}}{\partial r}-\frac{\alpha T}{\phi \mu^{2}} \frac{\partial \mu}{\partial w} \frac{\partial \lambda_{1}}{\partial z}-\frac{\alpha w}{\phi \mu^{2}} \frac{\partial \mu}{\partial w} \frac{\partial \lambda_{2}}{\partial z}+\left(2 \pi r \rho-\frac{\lambda_{3}}{\phi}\right) \frac{\alpha}{Z_{0} \bar{\mu}^{2}} \frac{\partial \bar{\mu}}{\partial \bar{w}} \frac{\partial \bar{w}}{\partial w}
\end{aligned}
$$

third co-state equation as:

$$
\frac{\partial \lambda_{3}}{\partial t}=0 ; \text { and }
$$


finally, the stationarity condition as:

$$
\begin{aligned}
\frac{\partial J}{\partial T_{\mathrm{int}}}= & -\frac{1}{R}\left[\gamma \frac{\partial \lambda_{1}}{\partial r}\right]_{R}-\frac{1}{Z_{0}}\left[\gamma \frac{\partial \lambda_{1}}{\partial z}\right]_{Z_{0}}+\frac{1}{Z_{0}}\left[\gamma \frac{\partial \lambda_{1}}{\partial z}\right]_{0} \\
& -\frac{1}{R}\left[D \frac{\partial \lambda_{2}}{\partial r}+\lambda_{2}\left(\frac{\partial D}{\partial w} \frac{\partial w}{\partial r}+\frac{\partial D}{\partial T} \frac{\partial T}{\partial r}\right)\right]_{R}\left(\frac{\partial w_{\mathrm{int}}}{\partial T_{\mathrm{int}}}\right) \\
& +\frac{\alpha \lambda_{3}}{\phi \bar{\mu}^{2} Z_{0}} \frac{\partial \bar{\mu}}{\partial \bar{w}}\left(\frac{\partial \bar{w}}{\partial w_{\mathrm{int}}}\right)\left(\frac{\partial w_{\mathrm{int}}}{\partial T_{\mathrm{int}}}\right)+\frac{\alpha \lambda_{3}}{\phi \bar{\mu}^{2} Z_{0}} \frac{\partial \bar{\mu}}{\partial \bar{T}}\left(\frac{\partial \bar{T}}{\partial T_{\mathrm{int}}}\right) \\
& -\frac{\beta r}{Z_{0} \cdot \bar{\mu}^{2}}\left[\frac{\partial \bar{\mu}}{\partial \bar{w}}\left(\frac{\partial \bar{w}}{\partial w_{\mathrm{int}}}\right)\left(\frac{\partial w_{\mathrm{int}}}{\partial T_{\mathrm{int}}}\right)+\frac{\partial \bar{\mu}}{\partial \bar{T}}\left(\frac{\partial \bar{T}}{\partial T_{\mathrm{int}}}\right)\right] .
\end{aligned}
$$




\section{Bibliography}

[1] Stuart Benett. A history of control engineering, 1979.

[2] Edward John Routh. On laplace's three particles, with a supplement on the stability of steady motion. Proceedings of the London Mathematical Society, 1(1):86-97, 1874.

[3] Stuart Bennett. A brief history of automatic control. IEEE Control Systems Magazine, 16(3):17-25, 1996.

[4] Nicolas Minorsky. Directional stability of automatically steered bodies. Journal of the American Society for Naval Engineers, 34(2):280-309, 1922.

[5] B. Wayne Bequette. Process control: modeling, design, and simulation. Prentice Hall Professional, 2003.

[6] George Stephanopoulos. Chemical process control: an introduction to theory and practice. 1984.

[7] Ramon Vilanova. Pid controller tuning rules for robust step response of first-order-plusdead-time models. In 2006 American Control Conference, pages 6-pp. IEEE, 2006.

[8] S. Abirami, H. Kala, P.B. Nevetha, B. Pradeepa, R. Kiruthiga, and P. Sujithra. Performance comparison of different controllers for flow process. International Journal of Computer Applications, 90(19):17-21, 2014.

[9] Mohammad Shahrokhi and Alireza Zomorrodi. Comparison of pid controller tuning 
methods. Department of Chemical \&3 Petroleum Engineering Sharif University of Technology, pages 1-2, 2013.

[10] Heikki N. Koivo and Anna Reijonen. Tuning of pid controllers for varying time-delay systems. In Proceedings of the IEEE International Conference on Mechatronics, 2004. ICM'04., pages 446-451. IEEE, 2004.

[11] Sahaj Saxena and Yogesh V. Hote. Advances in internal model control technique: A review and future prospects. IETE Technical Review, 29(6):461-472, 2012.

[12] Carlos E. Garcia and Manfred Morari. Internal model control. a unifying review and some new results. Industrial \& Engineering Chemistry Process Design and Development, 21(2):308-323, 1982.

[13] Evanghelos Zafiriou and Manfred Morari. Design of the imc filter by using the structured singular value approach. In 1986 American Control Conference, pages 1-6. IEEE, 1986.

[14] Carlos E. Garcia and Manfred Morari. Internal model control. 2. design procedure for multivariable systems. Industrial \& Engineering Chemistry Process Design and Development, 24(2):472-484, 1985.

[15] Jay H. Lee. Model predictive control: Review of the three decades of development. International Journal of Control, Automation and Systems, 9(3):415-424, 2011.

[16] J.L. Testud, J. Richalet, A. Rault, and J. Papon. Model predictive heuristic control: Applications to industial processes. Automatica, 14(5):413-428, 1978.

[17] Charles R. Cutler and Brian L. Ramaker. Dynamic matrix control: A computer control algorithm. In joint automatic control conference, number 17, page 72, 1980.

[18] David W. Clarke, Coorous Mohtadi, and P.S. Tuffs. Generalized predictive control—part i. the basic algorithm. Automatica, 23(2):137-148, 1987. 
[19] David W. Clarke, Coorous Mohtadi, and P.S. Tuffs. Generalized predictive control—part ii extensions and interpretations. Automatica, 23(2):149-160, 1987.

[20] J. D. Hedengren, R. A. Shishavan, K. M. Powell, and T. F. Edgar. Nonlinear modeling, estimation and predictive control in apmonitor. Computers \&5 Chemical Engineering, 70:133-148, 2014.

[21] Andrew Knyazev and Alexander Malyshev. Sparse preconditioning for model predictive control. In 2016 American Control Conference (ACC), pages 4494-4499. IEEE, 2016.

[22] A. Bemporad, M. Morari, V. Dua, and E. N. Pistikopoulos. The explicit linear quadratic regulator for constrained systems. Automatica, 38(1):3-20, 2002.

[23] Andrew Knyazev, Peizhen Zhu, and Stefano Di Cairano. Explicit model predictive control accuracy analysis. In 2015 54th IEEE Conference on Decision and Control (CDC), pages 2389-2394. IEEE, 2015.

[24] Martin Klaučo, Martin Kaluz, and Michal Kvasnica. Real-time implementation of an explicit mpc-based reference governor for control of a magnetic levitation system. Control Engineering Practice, 60:99-105, 2017.

[25] Sergio Lucia, Tiago Finkler, and Sebastian Engell. Multi-stage nonlinear model predictive control applied to a semi-batch polymerization reactor under uncertainty. Journal of Process Control, 23(9):1306-1319, 2013.

[26] G. Van Essen, M. Zandvliet, P. Van den Hof, O. Bosgra, and J.D. Jansen. Robust waterflooding optimization of multiple geological scenarios. SPE Journal, 14(01):202210, 2009.

[27] Thomas M. Stout and Theodore J. Williams. Pioneering work in the field of computer process control. IEEE Annals of the History of Computing, 17(1):6-18, 1995. 
[28] Carlos E. Garcia and Manfred Morari. Internal model control. a unifying review and some new results. Industrial \& Engineering Chemistry Process Design and Development, 21(2):308-323, 1982.

[29] Jay H. Lee, Manfred Morari, and Carlos E. Garcia. State-space interpretation of model predictive control. Automatica, 30(4):707-717, 1994.

[30] Kenneth R. Muske and James B. Rawlings. Model predictive control with linear models. AIChE Journal, 39(2):262-287, 1993.

[31] Alberto Bemporad and Carlo Filippi. Suboptimal explicit receding horizon control via approximate multiparametric quadratic programming. Journal of optimization theory and applications, 117(1):9-38, 2003.

[32] Tor A. Johansen, Idar Petersen, and Olav Slupphaug. Explicit sub-optimal linear quadratic regulation with state and input constraints. Automatica, 38(7):1099-1111, 2002.

[33] M. Diehl, H. G. Bock, J. P. Schlöder, R. Findeisen, Z. Nagy, and F. Allgöwer. Realtime optimization and nonlinear model predictive control of processes governed by differential-algebraic equations. Journal of Process Control, 12(4):577-585, 2002.

[34] Glauce De Souza, Darci Odloak, and Antônio C. Zanin. Real time optimization (rto) with model predictive control (mpc). Computers \& Chemical Engineering, 34(12):19992006, 2010.

[35] Rui Huang, Lorenz T. Biegler, and Sachin C. Patwardhan. Fast offset-free nonlinear model predictive control based on moving horizon estimation. Industrial $\mathcal{G}$ Engineering Chemistry Research, 49(17):7882-7890, 2010.

[36] Veronica Adetola and Martin Guay. Integration of real-time optimization and model predictive control. Journal of Process Control, 20(2):125-133, 2010. 
[37] Erdal Aydin, Dominique Bonvin, and Kai Sundmacher. Dynamic optimization of constrained semi-batch processes using pontryagin's minimum principle and parsimonious parameterization. In Computer Aided Chemical Engineering. Elsevier, 2017.

[38] S. Gros, M. Zanon, R. Quirynen, A. Bemporad, and M. Diehl. From linear to nonlinear mpc: bridging the gap via the real-time iteration. International Journal of Control, pages 1-19, 2016.

[39] Inga J. Wolf and Wolfgang Marquardt. Fast nmpc schemes for regulatory and economic nmpc-a review. Journal of Process Control, 44:162-183, 2016.

[40] J. Andrew Pruszynski and Stephen H. Scott. Optimal feedback control and the longlatency stretch response. Experimental brain research, 218(3):341-359, 2012.

[41] J.D. Pearson. Approximation methods in optimal control i. sub-optimal control. International Journal of Electronics, 13(5):453-469, 1962.

[42] Scott C. Beeler and David E. Cox. State-dependent riccati equation regulation of systems with state and control nonlinearities. 2004.

[43] S. Rafee Nekoo. Nonlinear closed loop optimal control: a modified state-dependent riccati equation. ISA transactions, 52(2):285-290, 2013.

[44] Lionel Mathelin, Luc Pastur, and Olivier Le Maître. A compressed-sensing approach for closed-loop optimal control of nonlinear systems. Theoretical and computational fluid dynamics, 26(1-4):319-337, 2012.

[45] Robert F. Stengel. Optimal control and estimation. Courier Corporation, 1994.

[46] Donald E. Kirk. Optimal control theory: an introduction. Courier Corporation, 2012.

[47] Simant Ranjan Upreti. Optimal control for chemical engineers. CRC Press, 2016. 
[48] Arthur Earl Bryson. Applied optimal control: optimization, estimation and control. Routledge, 2018.

[49] W. Harmon Ray. Advanced process control, 1982.

[50] Yu-Hong Dai. A perfect example for the bfgs method. Mathematical Programming, 138(1-2):501-530, 2013.

[51] Andrew P. Sage. Optimum systems control. Technical report, Southern Methodist University, 1968.

[52] H. Muhamad, S. R. Upreti, A. Lohi, and H. Doan. Optimal control study to enhance oil production in labscale vapex by varying solvent injection pressure with time. Optimal Control Applications and Methods, 37(2):424-440, 2016.

[53] Hadil Abukhalifeh, Simant Ranjan Upreti, and Ali Lohi. Effect of drainage height on concentration-dependent propane dispersion in vapex. The Canadian Journal of Chemical Engineering, 90(2):336-341, 2012.

[54] S.K. Booran. Optimal Control of Atmospheric Air Injection for Enhanced Oil Recovery. Phd thesis, Ryerson University, Canada, 2017.

[55] Hadil Abukhalifeh, Ali Lohi, and Simant Ranjan Upreti. A novel technique to determine concentration-dependent solvent dispersion in vapex. Energies, 2(4):851-872, 2009.

[56] Simant R. Upreti. Process Modeling and Simulation for Chemical Engineers: Theory and Practice. John Wiley \& Sons, 2017. 